\title{
Novel radiopaque biomaterials for spinal surgery
}

Citation for published version (APA):

Boelen, E. J. H. (2007). Novel radiopaque biomaterials for spinal surgery. [Doctoral Thesis, Maastricht University]. Datawyse / Universitaire Pers Maastricht. https://doi.org/10.26481/dis.20070404eb

Document status and date:

Published: 01/01/2007

DOI:

10.26481/dis.20070404eb

Document Version:

Publisher's PDF, also known as Version of record

\section{Please check the document version of this publication:}

- A submitted manuscript is the version of the article upon submission and before peer-review. There can be important differences between the submitted version and the official published version of record.

People interested in the research are advised to contact the author for the final version of the publication, or visit the DOI to the publisher's website.

- The final author version and the galley proof are versions of the publication after peer review.

- The final published version features the final layout of the paper including the volume, issue and page numbers.

Link to publication

\footnotetext{
General rights rights.

- You may freely distribute the URL identifying the publication in the public portal. please follow below link for the End User Agreement:

www.umlib.nl/taverne-license

Take down policy

If you believe that this document breaches copyright please contact us at:

repository@maastrichtuniversity.nl

providing details and we will investigate your claim.
}

Copyright and moral rights for the publications made accessible in the public portal are retained by the authors and/or other copyright owners and it is a condition of accessing publications that users recognise and abide by the legal requirements associated with these

- Users may download and print one copy of any publication from the public portal for the purpose of private study or research.

- You may not further distribute the material or use it for any profit-making activity or commercial gain

If the publication is distributed under the terms of Article $25 \mathrm{fa}$ of the Dutch Copyright Act, indicated by the "Taverne" license above, 
Novel Radiopaque Biomaterials for Spinal Surgery 
Novel Radiopaque Biomaterials for Spinal Surgery

ISBN 978-90-5278-613-1

Printed by: Datawyse Maastricht

Cover design: Erik Boelen

(C) Erik Boelen, Maastricht 2007 


\title{
Novel Radiopaque Biomaterials for Spinal Surgery
}

\author{
PROEFSCHRIFT \\ ter verkrijging van de graad van doctor \\ aan de Universiteit Maastricht, \\ op gezag van de Rector Magnificus, \\ Prof. mr. G.P.M.F. Mols, \\ volgens het besluit van het College van Decanen, \\ in het openbaar te verdedigen \\ op woensdag 4 april 2007 om 16.00 uur \\ door
}

Erik Johannes Herman Boelen

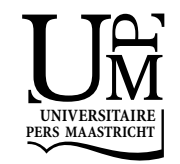




\section{Promotores}

Prof. dr. L.H. Koole

Prof. dr. S.J. Bulstra (Rijksuniversiteit Groningen)

\section{Copromotor}

Dr. C.S.J. van Hooy-Corstjens

\section{Beoordelingscommissie}

Prof. dr. R.G.T. Geesink (voorzitter)

Prof. dr. P. Brink

Prof. dr. J.M.A. van Engelshoven

Dr. Ir. L.E. Govaert (Technische Universiteit Eindhoven)

Prof. dr. J.A. Put (Universiteit Hasselt)

This research forms part of the research program of the Dutch Polymer Institute (DPI), project \#398. 


\section{Contents}

$\begin{array}{lll}\text { Chapter } 1 & \text { General Introduction } & 7\end{array}$

Chapter 2 Intrinsically Radiopaque Hydrogels for Nucleus 33 Pulposus Replacement

Chapter 3 Preliminary Evaluation of New Intrinsically 55 Radiopaque Hydrogels for Replacing the Nucleus Pulposus

Chapter 4 Towards a Functional Radiopaque Hydrogel for 69 Nucleus Pulposus Replacement

Chapter 5 Evaluation of a Highly Radiopaque Iodine91 Containing Acrylic Bone Cement for Use in Augmentation of Vertebral Compression Fractures

Chapter 6 General Discussion

Chapter 7 Appendix

Chapter 8 Summary/Samenvatting

List of Publications

Curriculum Vitae

Dankwoord 

General Introduction 
8 | Chapter 1 


\section{Introduction}

Back pain recognizes no age, economic or ethnic barriers [1]. During the last four years, doing research leading to this thesis, I myself was struck by back pain once. It wore off after some weeks, but it really showed me how important your back is in everyday life. Also it has encouraged me to do this research. It was staggering how many people, as soon as they heard about my research on back pain, asked me for advice on their own back pain or on the back pain of a loved one. The most prominent and telling example is the e-mail (see Appendix 1 and the quote on this page) which I received from a man in Scotland, after he found and read on the internet, the article

"I am desperate to regain my ability to swim, cycle and run... ... and willing to offer myself as a patient for use in clinical trials.

Jim Scanlan described in Chapter 3. As I am not a physician, it grieved me not being able to help the people who came to me for advice, to get rid of their debilitating back pain. However, I hope this work will be continued in order to make a contribution to the management of back pain and to provide orthopaedic surgeons with engineered polymeric biomaterials as viable alternatives in combating back pain.

\section{Epidemiology}

Back pain is a considerable health problem in all developed countries, with a lifetime prevalence of about $70 \%$ and an average point prevalence of 30\% [24]. In effect, it is the largest cause of activity limitation in people younger than 45 years, a major cause of work absenteeism and one of the most frequent reasons for visiting a physician or physical therapist [4-6]. It could be said that back pain is a universal epidemic. The impact of back pain on society is usually estimated by examining the costs. In the Netherlands in 2000, the direct medical costs of back pain were estimated to be 337.3 million euros, $0.9 \%$ of the total medical costs [3]. However, the indirect costs, e.g. production losses due to work absenteeism and disablement, are much higher and were estimated in 1991 to consist of $93 \%$ of the total costs of back pain [5]. Assuming an equal ratio in 2000 , this would mean a total cost of back pain of 
4.8 billion euros, which corresponds to $1.1 \%$ of the gross national product in that year [7]. Moreover, the ageing of the population will result in a strong increase in the amount of ailments to the locomotor apparatus, of which the back is an important participant. Because of the large impact of these ailments on society, the growing severity of the situation is recognized internationally and the years 2000-2010 are proclaimed as The Bone and Joint Decade [8]. One of the primary goals is to raise awareness of the growing burden of musculo-skeletal disorders on society. As a part of this initiative, in the Netherlands the year 2007 is declared the year of back and neck pain. Besides the economic impact, back pain evidently also has a huge psychological and social impact $[4,9]$. The reverse is also true, i.e. social and psychological aspects have an unmistakable influence on back pain [10].

In general, people experiencing acute back pain will see their pain and related disability resolve in a few weeks time, but recurrences are common. The severity of these recurrences is usually less and hence does not always lead to a new visit to the physician. Only a small proportion (5\%) of people with an acute episode of back pain develop chronic back pain (pain that persists for over 3 months) [2]. In most cases, back pain occurs in the low back since this part carries the most (body)weight and the largest moments [11].

From all the people suffering from back pain and who consult a physician, only about $10 \%$ receive a specific diagnosis, like hernia nucleus pulposus (HNP, \pm $5 \%)$ among others. Non-specific back pain is defined as symptoms with no clear specific cause, which is the case in the other $90 \%$ of people with back pain $[2,3]$. In the Netherlands 44,600 people with acute back pain were diagnosed with HNP in the year 2000. It is a common misconception that radiographs and newer imaging techniques, like computed tomography (CT) and magnetic resonance imaging (MRI) can always identify the cause of pain [12].

Another ailment of the back, occurring more and more are vertebral compression fractures $[13,14]$. Especially postmenopausal women are vulnerable for this pathology, affecting approximately $25 \%$ of women over 50 years of age [15]. Osteoporosis lies at its base; it weakens the vertebral bodies and these can fracture under (excessive) loading [16]. Osteoporosis is associated with age and as a result vertebral compression fractures are expected to become a growing problem due to the ageing of the population. 


\section{The spinal motion segment}

The human spine is designed around the spinal cord, which lies at its center. The spine has at least three fundamental biomechanical functions: (i) it transfers the weights and bending moments of the head and the trunk to the pelvis, (ii) it allows sufficient physiological motion between these three body parts and (iii) most importantly, it protects the spinal cord from potentially damaging forces or motions produced by trauma [11].
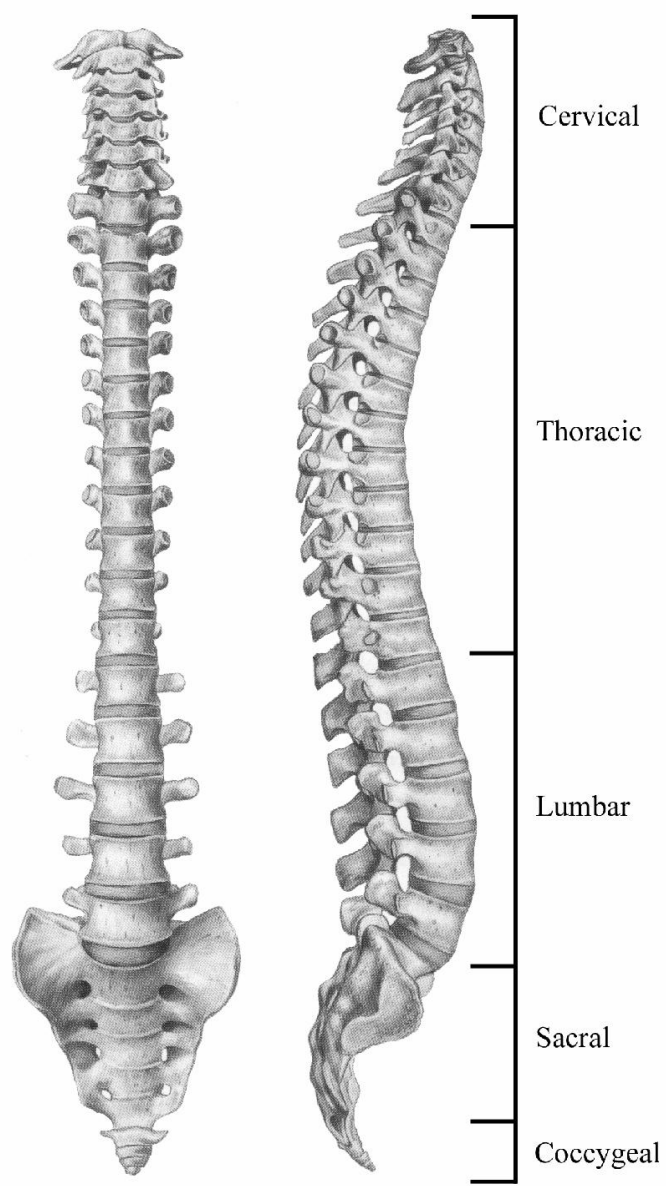

Figure 1: The human spine and its regions, front and lateral view.

The spine consists of seven cervical vertebrae, twelve thoracic vertebrae, five lumbar vertebrae, five fused sacral vertebrae and three to four fused coccygeal vertebrae (Figure 1). The vertebrae are numbered from top to bottom: cervical 
C1-C7, thoracic T1-T12, lumbar L1-L5, sacral S1-S5 and coccygeal Co1-Co4. Except for the upper two cervical vertebrae, i.e. the atlas and the axis, the cervical, thoracic and lumbar vertebrae are separated by flexible intervertebral discs. Because of their flexibility, the discs allow the spine to twist and bend so that the body is able to assume a wide range of postures. It is often supposed that their primary function is to act as shock absorbers, but there is considerable evidence against this supposition: (i) the disc does not act as a shock absorber during in vitro mechanical tests; (ii) shock absorption is normally achieved by the musculature; (iii) there is no reason why the spine needs to be regularly punctuated by shock absorbers any more than do the other long bones. The discs act as joints, which are responsible for the essential flexibility of the spine to perform its function as the main component of the axial skeleton [17]. Along the length of the spine there are also various ligaments that protect the spinal cord by restricting motions within well-defined limits [11]. A spinal part consisting of two adjacent vertebrae with the intervertebral disc in between is called a motion segment (Figure 2). The motion segment has three joints; the intervertebral disc and two facet joints.

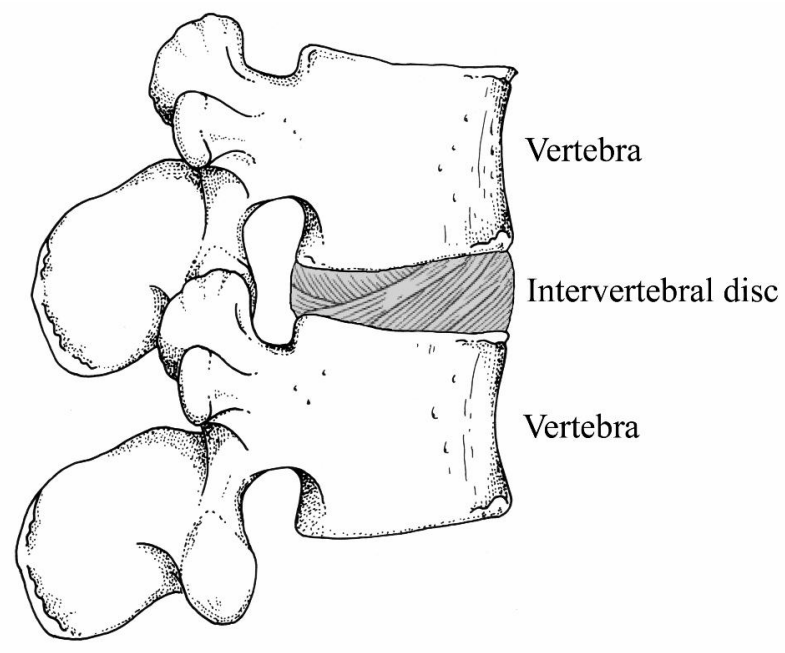

Figure 2: The spinal motion segment consisting of two adjacent vertebrae and the intervertebral disc in between.

\subsection{The vertebra}

The human vertebrae increase in size from $C 1$ to $L 5$, as a mechanical adaptation to the progressively increasing loads to which they are subjected. The basic structure however is the same; it consists of an anterior block of 
bone, the vertebral body, and a posterior bony ring, the neural arch (Figure 3). This arch contains articular (facet joints), transverse and spinous processes. The spinal cord runs through the neural arches of the vertebrae. The vertebral body is a roughly cylindrical mass of cancellous bone, surrounded by a thin shell of cortical bone. Its superior and inferior surfaces are slightly concave and accommodate the cartilaginous end-plates. Only the fused sacral and coccygeal vertebrae are basically different [11].

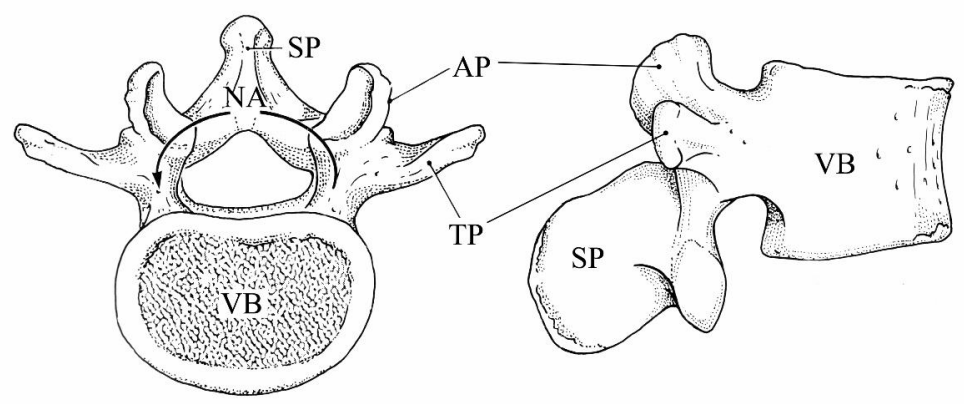

Figure 3: Typical lumbar vertebra, top view (left) and lateral view (right). (VB vertebral body, NA - neural arch, AP - articular process, TP - transverse process, $S P$ - spinous process).

\subsection{The intervertebral disc}

The human intervertebral disc is roughly cylindrical in cross-section, but at the lumbar level the discs are more kidney-bean shaped. Overall, their anterior height is greater than their posterior height, so they appear wedge shaped in sagittal section. The average thickness of a lumbar disc is about $10 \mathrm{~mm}$. The intervertebral disc has a soft, gel-like inner region, the nucleus pulposus (or nucleus), that fills $30-50 \%$ of the disc cross-sectional area, surrounded by a tough, fibrous outer region, the annulus fibrosus (or annulus) (Figure 4).

The annulus consists of lamellae of oriented collagen fibrils, enclosing the nucleus like a tube. Although the nucleus and annulus are two distinct structures, the boundary between them is not clearly defined; there is a gradual transition from the mucoid texture of the nucleus to the laminated structure of the annulus. The upper and lower surfaces of the disc consist of thin ( $\pm 1 \mathrm{~mm}$ ) hyaline cartilage end-plates that separate the disc from the vertebral bodies. 


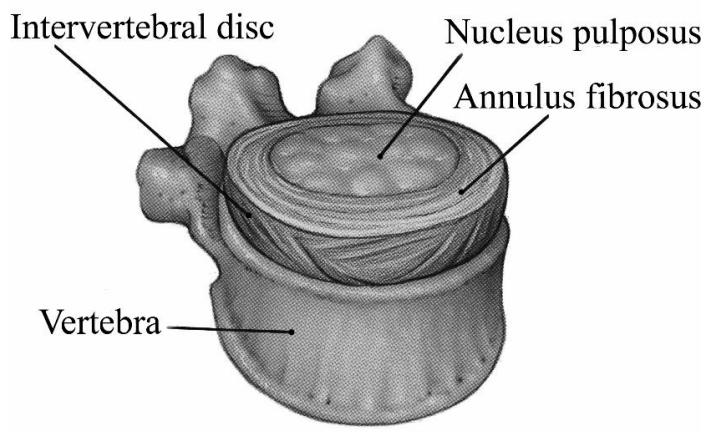

Figure 4: The intervertebral disc.

The inner lamellae of the annulus merge into the end-plates to form a closed vessel containing the nucleus, whereas the outer lamellae are attached to the bone of the adjacent vertebral bodies (Figure 5) [11, 17, 18].

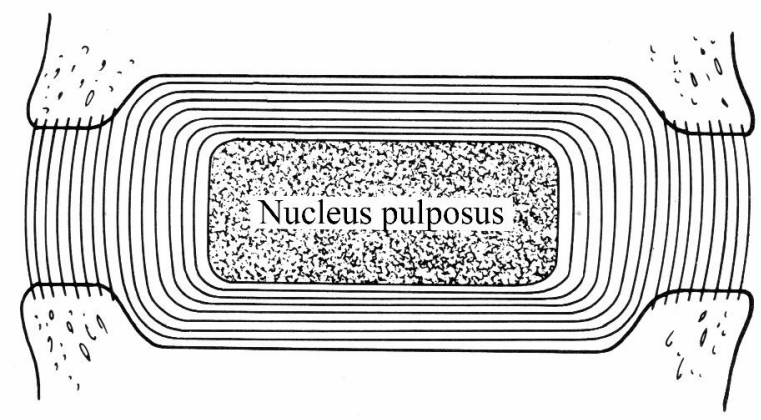

Figure 5: Exaggerated sagittal view of the vertebral end-plates and the laminated structure of the annulus fibrosus.

The nucleus pulposus consists of a highly hydrated gel of proteoglycans, which in turn consist of sulphated glycosaminoglycan side-chains covalently bound to core protein. Also some collagen (mostly type II) and few cells are present. Some of the proteoglycans aggregate by interaction of the protein core with hyaluronic acid. The sulphate and carboxyl groups of the glycosaminoglycans are negatively charged and are not free to diffuse out of the matrix, since they are part of a high molecular weight polymer. This leads to a high density of fixed negative charges. The fixed negative charges are electrostatically balanced by mobile cations, e.g. $\mathrm{Ca}^{2+}$. Due to the elevated levels of mobile ions compared to its surroundings, the nucleus has a higher osmotic pressure and as a result, the matrix attracts water. In children and young adults, the water content of the nucleus exceeds $80 \%$. The water content decreases with age, 
due to compositional changes of the nucleus. The high water content makes the nucleus easy to visualize using $T_{2}$-weighted MRI [19].

The annulus fibrosus is made up from about a dozen concentric layers of collagen fibres, the lamellae. In each lamella the fibres are parallel and tilted about 30 degrees with respect to the disc plain; the successive lamellae are tilted in opposite directions (Figure 6). Hence, adjacent bands are at 60 degrees to each other. The inner annulus contains mainly collagen type II, whereas in the outer annulus collagen type I is predominantly present. The lamellae are thick in the anterior and lateral portions of the annulus, but posteriorly they are finer and more tightly packed. Consequently, the posterior region is thinner than the rest of the annulus.

The cartilaginous end-plates are composed of hyaline cartilage, which looks like the articular cartilage of synovial joints, and fibrocartilage.

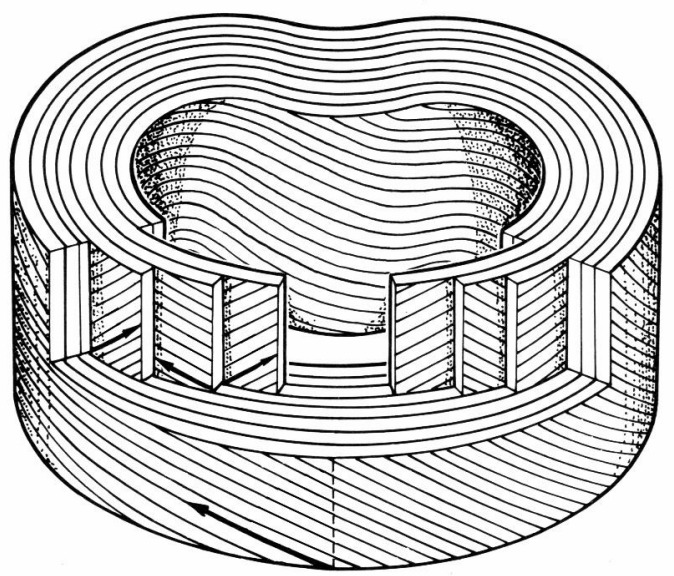

Figure 6: Detailed structure of the fibre orientations in the lamellae of the annulus fibrosus; the 30 degree angle with the disc plane is the same, but opposite in consecutive lamellae.

The intervertebral disc is the largest organ in the body without blood supply, so it depends on diffusion through the end-plates and annulus from the surrounding blood vessels, for nutrient supply and the removal of metabolic waste products. Furthermore, by loading and unloading the disc, convective transport of larger solutes occurs through a kind of pumping action [20-22].

\subsection{Disc function}

As a result of the osmotic pressure in the nucleus, enclosed by the annulus and end-plates, the intervertebral disc is continuously pressurized. This pressure, 
the swelling pressure, in healthy discs about $0.1 \mathrm{MPa}$ [23], is balanced axially by muscle and ligament tension. It also has a radial component, exerting pressure on the annulus, in which it is balanced by tension in the annular fibres. The swelling pressure makes the disc able to support loads, like compressed air in a tire. This mechanism is especially efficient in resisting axial loads, which is the main loading direction in the spine $[11,17,18]$. When an axial load is applied, the nucleus acts as an incompressible, isotropic fluid, distributing the force evenly in all directions; pushing the annulus outward and the end-plates apart. Hence, much of the load is transferred radially to the annulus fibres, which are stretched as a result (Figure 7). The orientation of the annular fibres is important in the transfer of loads from one vertebra to the other. Since the collagen fibres are elastic, they store the energy needed to stretch them. If the applied load is released, the elastic recoil of the collagen fibres exerts the stored energy back onto the nucleus and the initial disc height is restored.

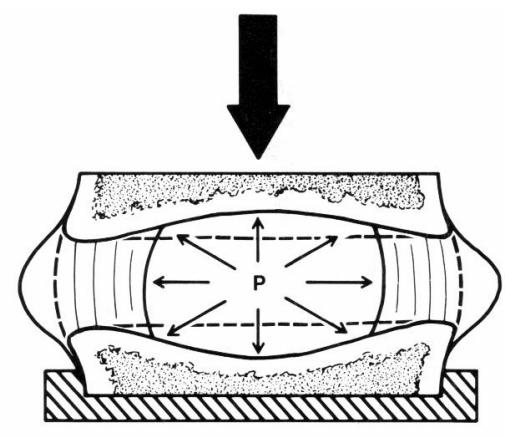

A

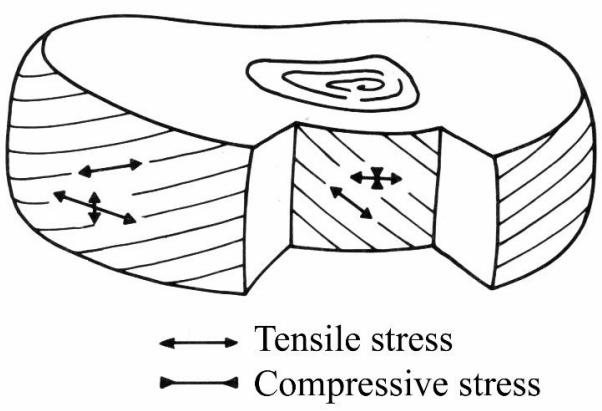

$\mathrm{B}$

Figure 7: Intervertebral disc under axial compression, (A) The increased pressure in the nucleus pushes the annulus and end-plates outward, resulting in annulus bulging and end-plate deflection, $(B)$ the stresses in the annulus vary in different directions and at different depths.

However, the disc is no perfectly sealed container and during continuous loading some fluid is pressed out, presumably in order to dissipate some of the potentially damaging compressive energy. Consequently, the disc exhibits viscoelastic behaviour, like creep and hysteresis. For example, during the day, the discs are continuously loaded and some fluid is lost. During the night, the discs reswell. As a result people are on average $2 \mathrm{~cm}$ taller in the morning than in the evening [24].

Other loads the disc has to cope with besides axial compression are tension, torsion, bending and shear. Primarily, the oriented fibrous structure of the 
annulus is very important in these loading profiles. Off course, the in vivo loads of the spine are very complex and are mostly a combination of these loads. In short, the discs allow motion of the spine and transfer loads between the vertebrae.

\section{Back pain}

Back pain has a myriad of causes, e.g. mechanical, congenital, inflammatory, degenerative, but mostly no definite cause-effects can be identified ( $90 \%$ has non-specific back pain). Interestingly, psychosocial factors, including insurance benefits, have been demonstrated to be more important than biomechanical workload [9]. The intervertebral disc is considered to be a major contributor in specific back pain [19]. Back pain occurs most frequently in the lumbar range. So the lumbar intervertebral disc is one of the foci of this work. Pain occurs mainly by physical, chemical or inflammatory innervation of the nerve roots, branching from the spinal cord, possibly also giving the sensation of leg pain. A plethora of pathologies of the intervertebral disc can possibly lead to pain, but in this work the focus is on the bulging or herniated disc, in early stages of degeneration, which is a pathology that is readily detectable by MRI.

As we age, the nucleus becomes more fibrous and the glycosaminoglycan content decreases. This process is called degeneration. As a result, the nucleus becomes desiccated and the disc loses height. The boundary between nucleus and annulus becomes even less distinct. Loss of disc height puts relatively more axial load on the annulus fibrosus, which can weaken it. This can lead eventually to annular bulging or even a herniation of the nucleus through a defect in the annulus. The annulus can be partly or completely ruptured. Disc bulge is also referred to as disc protrusion, and herniation is often called disc prolapse (partial annulus rupture) or extrusion (complete herniation through the annulus). Mostly, disc bulge or herniation occurs posteriorly, since the annulus is thinnest in that region. Pure axial loading does not lead to annular defects, instead a combination of torsion and bending does the damage [11, 18]. The bulging annulus or herniated nucleus can irritate the neighbouring nerves, causing pain (Figure 8).

Another cause of disc bulge or herniation is trauma. A short heavy load, especially in a flexed posture, can cause herniation of the nucleus through the annulus. Mostly, the disc is already somewhat weakened by torsional injuries 
[18]. In younger persons, when the nucleus is still turgescent, the symptoms are likely to be more dramatic [19].
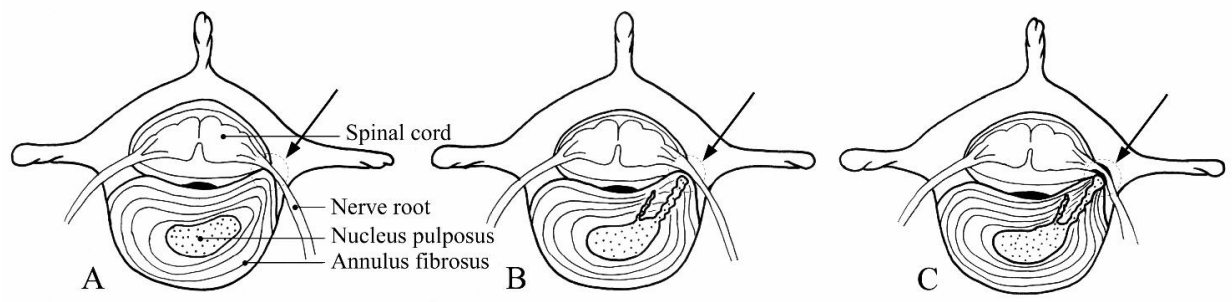

Figure 8: Disc pathologies, (A) bulging disc, (B) disc prolapse and (C) extrusion, all causing nerve root irritation (arrow).

Back pain can also be due to a vertebral compression fracture. The vertebral body can collapse under excessive loading, like in a traumatic car accident, or when the bone density is decreased by osteoporosis (Figure 9). Depending on the severity of the osteoporosis, the cause of the fracture can be simple, such as vigorous sneezing [16]. Vertebral compression fractures occur most often at the lower thoracic and higher lumbar levels, being a transition zone between the relatively stiff thoracic and more mobile lumbar segments [25].

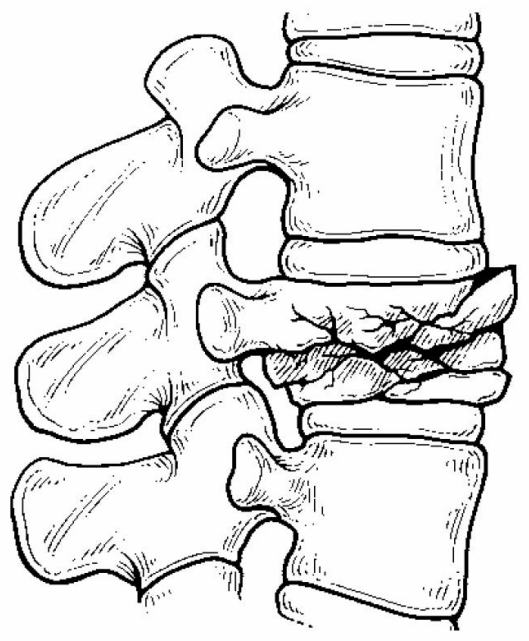

Figure 9: Vertebral compression fracture. 


\section{Treatment options}

\subsection{Disc problems}

People experiencing low back pain, due to disc problems and severe enough to consult a physician, have several treatment options; initially an array of conservative treatments, or various surgical procedures. Conservative treatment includes: exercise, physical therapy, anti-inflammatory drugs, painkillers, and muscle relaxants among others, although the efficacy of these treatments remains a point of debate $[2,10,26]$. If conservative methods do not suffice to alleviate the pain, people usually undergo surgery. In the Netherlands, about 11,000 hernia operations are performed annually, which contributes to having the second highest ratio for back surgery in the world, after the US [27, 28]. Several surgical procedures for the treatment of back pain will now be discussed:

\section{- (Partial) discectomy or nucleotomy}

This procedure involves the removal of (part of) the affected intervertebral disc or nucleus pulposus in an attempt to relieve nerve root compression [10]. Nowadays, the procedure can be performed by minimally invasive techniques; endoscopically and percutaneously. The primary goal is to alleviate pain and as such it does not restore the function of the disc. An alternative technique is chemonucleolysis, in which the nucleus is chemically dissolved by the local injection of the enzyme chymopapain. However, this alternative method is still the subject of controversy. The results of discectomy for pain relief are good to excellent, with a success rate of $70 \%$ to $95 \%$ in carefully selected patients. Still, discectomy introduces mechanical instability and was found to lead to a loss of disc height $[29,30]$.

\section{- Spinal fusion}

Spinal fusion is widely performed in the management of back pain $[11,31]$. It is mostly done in combination with a total discectomy. The rationale is to alleviate pain by diminishing movement between two vertebrae and restoring disc height. In this procedure, the spinal motion segment is immobilized by fusing the adjacent vertebrae together. Fusion is achieved by mechanical fasteners (screws and rods) and/or bone grafts, supplemented potentially with a cage (Figure 10A). Although this technique is widely used, there are conflicting reports on its effectiveness $[2,10]$ and many concerns about the effect on the 
adjacent levels [32-35]. The adjacent levels will have to compensate for the loss of motion in the fused segment.

\section{- Total disc replacement}

After a total discectomy, a synthetic artificial disc is placed in between the vertebrae (Figure 10B). The underlying principle is to maintain/restore the mobility of the segment on top of the pain alleviation. In effect, it is a prosthetic joint. There are many designs, few of which have made it to clinical pratice, even less were somewhat succesfull [36-38]. Examples of designs are: a solid steel ball (earliest design) [39], all-polymeric prosthesis [40], three-dimensional woven fabric [41], ultra-high-molecular-weight polyethylene nucleus in between metal end-plates [42] and many, many more. The problems that occur most often are difficulties of anchoring the prosthesis in the vertebral bodies, associated with implant subsidence, and the formation of wear particles capable of eliciting an immune response [43-45]. Overall, the effectiveness is comparable to a fusion procedure $[36,46,47]$.

\section{- Nucleus replacement}

In view of the fact that it has proved to be extremely complicated to replace an entire intervertebral disc and recognizing that it is not always necessary to remove the annulus fibrosus, and desirable to retain the functions of the nucleus pulposus, the idea arose to replace only the nucleus pulposus [48-50]. The troublesome nucleus is removed and replaced with a prosthesis (Figure 10C). Nucleus replacements also come in a wide variety of designs [51-54], but the most promising and most investigated designs consist of a hydrogel. The main advantage of hydrogels is their capability to absorb water, which resembles the natural nucleus. This technique as yet has not been introduced into routine clinical practice, but is a promising future alternative. For it to be useful, it is important that the annulus is still functional. Device migration or extrusion are the largest concerns with this procedure [55-57].

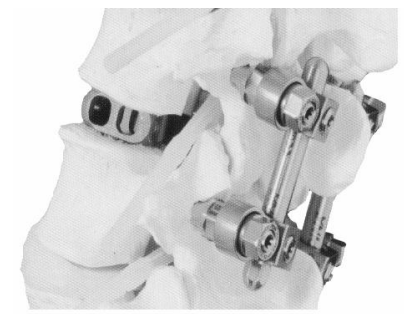

A

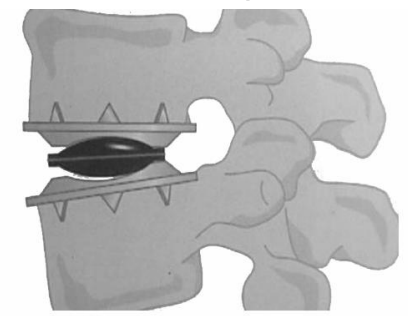

$\mathrm{B}$

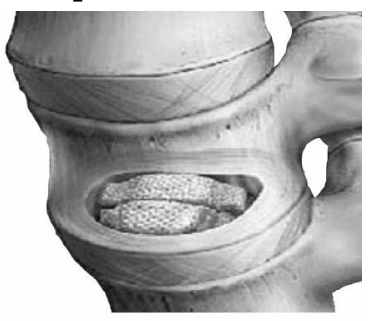

$\mathrm{C}$

Figure 10: Examples of treatment options, (A) spinal fusion, (B) total disc replacement and $(C)$ nucleus replacement. 


\section{- Tissue engineering}

This approach is still in its infancy. Attempts are made to regenerate the natural disc. A cellular graft is inserted to stimulate natural repair [50,58]. The technique seems promising, but for this approach to be successful, there are still a lot of hurdles to overcome and moreover, if the nutrition of the disc is hampered, insertion of cells will be useless [59-62].

\subsection{Vertebral compression fractures}

For people suffering from symptomatic vertebral compression fractures, secondary to osteoporosis, the focus of treatment is pain alleviation. Initially this is done with analgesics. If necessary, the height and stability of the affected vertebra can nowadays be restored by either vertebroplasty or balloon kyphoplasty (Figure 11). Both procedures involve the percutaneous injection of bone cement into the vertebral body. In kyphoplasty the cement is injected after the inflation of a balloon in order to augment the vertebral body and create a space for the cement. Success rates for these procedures exceed $90 \%$ $[63,64]$.

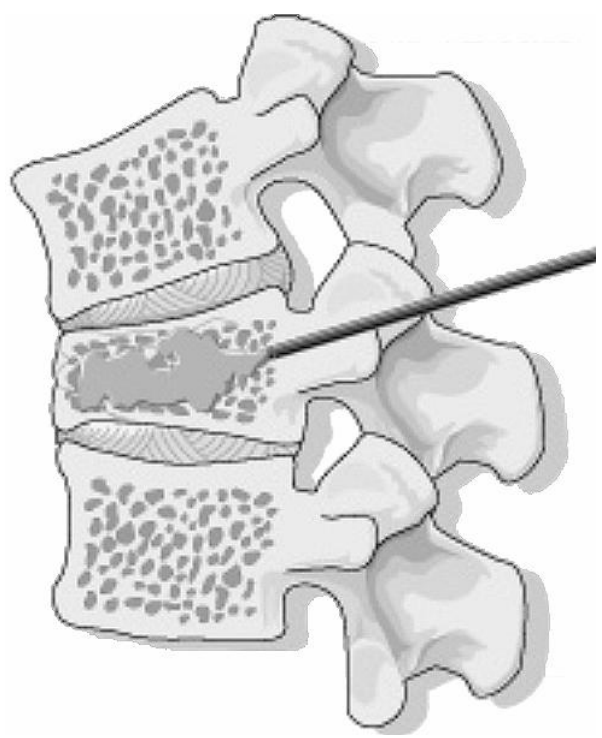

Figure 11: The injection of cement into a collapsed vertebral body during a vertebroplasty procedure. 


\section{Our approach}

\subsection{Disc problems}

The greatest challenge for a successful surgical procedure, especially for the long-term, is to retain the motion in the motion segment, but also the stability. This contradictory demand is what makes the natural intervertebral disc such a complex joint, and hence so difficult to replace. From the surgical procedures for the management of disc problems described above, we feel the nucleus replacement holds most promise, and especially the hydrogel nucleus replacement. The orthopaedic surgeons from the Academic Hospital in Maastricht, who are often faced with the ill-effects of total disc replacement in particular $[43,65,66]$, suggested the development of a novel type of hydrogel nucleus prosthesis. On top of the obvious demands for a nucleus prosthesis, vide infra, they wanted the prosthesis to be intrinsically radiopaque with X-ray fluoroscopy. To take up the challenge of designing an intrinsically radiopaque hydrogel, we built on our knowledge of intrinsically radiopaque polymers [67] and hydrophilic coatings [68].

As a start it is important to realize the demands for a nucleus prosthesis. However, to date there are no official standards describing a nucleus replacement, but some requirements have been suggested by Bao [48, 69]. Based on these papers, some other literature sources [49, 51, 54, 70] and our own insights, we stipulated the following demands:

1) The nucleus prosthesis should be intrinsically radiopaque and compatible with MRI. Intrinsic radiopacity allows for complete visualization using X-rays. This aids the surgeon in positioning the implant in the nucleus cavity. It is also useful to evaluate the implant at a later time.

2) The nucleus prosthesis can be implanted through a small incision in the annulus fibrosus and fills the entire nucleus cavity after implantation. Fortunately, this is an inherent advantage of a hydrogel; it can be implanted in the dry state, called xerogel, and swell in situ to fill the entire nucleus cavity and restore disc height. Cavity conformity is essential to transfer compressive loads to the annulus and distribute forces over the entire end-plates.

3) Once in place, the nucleus prosthesis should take over the function of the natural nucleus. The stiffness of the prosthesis material should be tuned, to mimic the response of the pressurized fluid under loading. When compressed, it should expand radially to transfer the compressive force to the annulus. The prosthesis material should be elastic and recover its height after the load is 
removed. It should also be fatigue resistant and preferably resume the pumping action of the natural nucleus pulposus.

4) As for any material used in the human body, the material for the nucleus prosthesis should be biocompatible.

Successful implementation of this technique also requires appropriate patient selection, hence proper indications and contraindications are important. No formal indications are formulated so far, but suggestions are made in literature $[48,51,54]$. Nucleus replacements can be used as an adjunct to discectomy, since it is designed to address the pitfalls of discectomy, without losing its benefits. It can be used as an alternative in patients who require fusion, but who still have a tall and stable disc, which are mainly young people in early stages of degeneration, or people who have experienced a traumatic herniation. Contraindications include advanced degeneration with disc height less than 5 $\mathrm{mm}$ and an incompetent annulus, spondylolisthesis (forward slippage of one vertebra over another) and Schmorl's nodes (herniations of the nucleus through the end-plates). Practically, the indications are dictated by benefit-to-cost and benefit-to-risk ratios, compared to discectomy and fusion. The risk for a nucleus replacement is slightly higher than for discectomy alone, but less than for fusion. Extra costs are associated with a nucleus replacement over a discectomy alone.

As mentioned earlier, there are many designs for nucleus replacements. None of the designs is commercially available in the USA yet and none so far are approved by the Food and Drug Administration (FDA) [48, 54]. In Europe however, one design is already used clinically, while others are in clinical trials. The most prominent examples are the Prosthetic Disc Nucleus (PDN), developed by Raymedica Inc. (Bloomington, Minnesota, USA) [71] and the Newcleus, developed by Sulzer Spine-Tech (Edina, Minnesota, USA) [52] (Figure 12). The PDN consist of a polyacrylamide based hydrogel core, surrounded by a polyethylene jacket. The Newcleus is a memory coiling spiral made from polycarbonate urethane. The PDN has the largest human clinical experience. The design and implantation procedure were adapted after initial problems of device migration and end-plate damage [51, 72, 73]. The PDN has helped to elucidate the promises and pitfalls of nucleus replacements in general and has served as a guiding concept for the development of many nucleus prostheses. 

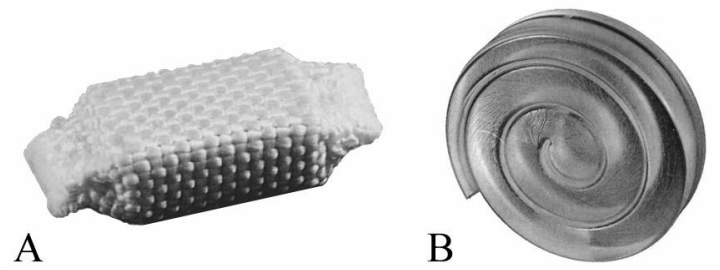

Figure 12: Examples of nucleus replacements, $(A)$ the Prosthetic Disc Nucleus $(P D N)$ and $(B)$ the memory coiling spiral (Newcleus).

We feel that both the PDN and Newcleus can be improved in terms of radiopacity and cavity conformity; the PDN is not shaped to fit the kidney-bean shaped nucleus cavity exactly and relies on metallic markers for radiographic visualization, and the Newcleus is also not a perfect fit and is radiolucent (Figure 13).

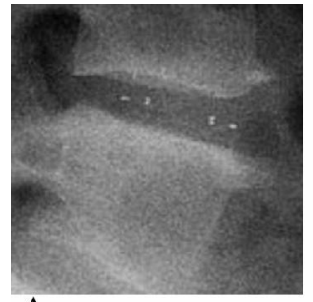

A

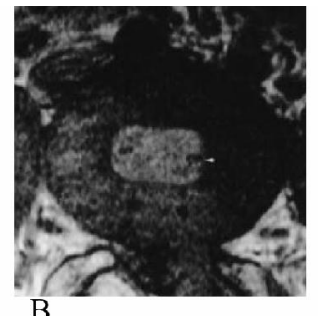

$\mathrm{B}$

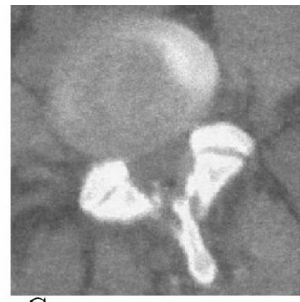

$\mathrm{C}$

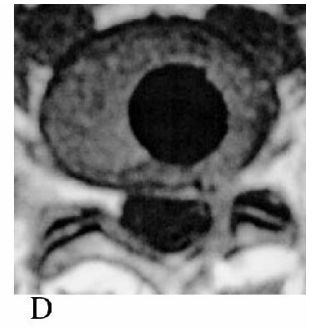

Figure 13: Visualization of nucleus replacements using X-Ray and MRI, (A) plain X-ray image of the PDN in between two vertebrae showing small radiopaque markers, (B) axial MRI of the PDN in situ, (C) axial CT-image of an intervertebral disc containing the radiolucent Newcleus and (D) axial MRI of the Newcleus in situ.

The concerns for a nucleus prosthesis, being implant migration and extrusion can potentially be avoided by cavity conformity of the prosthesis and repair of the annulus. For annulus repair, a tissue engineering strategy might be employed, however this falls outside the scope of this thesis.

\subsection{Vertebral compression fractures}

Acrylic bone cements used in total joint replacements are made radiopaque by the addition of inorganic contrast agents, with its inherent drawbacks. Bone cements consist of a liquid and a powder, which are mixed before use [74]. Acrylic monomer and accelerator constitute the liquid phase, and polymer microspheres, the initiator benzoyl peroxide (BPO) and contrast agent represent 
the powder phase. Mixing both phases initiates polymerization and consequent hardening of the cement by combining initiator and accelerator. The polymer microspheres slightly dissolve and become part of the polymerizing matrix. The inorganic contrast agents just reside within the matrix and have a tendency to form lumps. These lumps are known to act as crack-initiation sites [75].

Also in vertebroplasty and kyphoplasty, these radiopaque acrylic bone cements are mostly used. However, the required X-ray contrast is higher for these procedures for improved fluoroscopic visualization. This is achieved by increasing the amount of inorganic contrast agents, with its concomitant disadvantages. For total joint replacements the amount of inorganic contrast agent in the powder component of the cement is $10-15 \mathrm{wt} . \%$, whereas for vertebroplasty and kyphoplasty $30 \mathrm{wt} . \%$ must be used [76]. With our existing knowledge on intrinsically radiopaque bone cement, we also hope to develop a viable alternative for the augmentation of vertebral compression fractures. When using acrylic bone cement for these applications, besides the elevated contrast level, the cement needs to be less viscous for injection through a 1015 gauge needle $[77,78]$, and it needs to have a longer working time. Commonly this is achieved by increasing the liquid-to-powder ratio $[79,80]$ and decreasing the amount of initiator.

\subsection{Intrinsic radiopacity}

The X-ray absorption of a material at a certain photon energy is dependent on its thickness, density and elemental composition, as is clear from equation 1 , which relates the $X$-ray intensity leaving the object $(I)$ to the incident intensity $\left(I_{o}\right)$, at a single photon energy [81, 82]:

$$
I=I_{o} \cdot e^{[-(\mu / \rho) x]}
$$

in which $x$ is the material mass thickness and $(\mu / \rho)$ is the mass attenuation coefficient ( $\mu$ is the linear attenuation coefficient and $\rho$ is the density). This coefficient tends to increase with increasing atomic mass. Absorption is mainly achieved by the collision of the X-ray photons with the electrons in a material. Hence, the absorption is higher when the concentration of electrons is higher. Elements with high atomic numbers have high electron densities. Water, with the lower elements $\mathrm{H}$ and $\mathrm{O}$, has low X-ray absorption, so any structure containing a significant amount of it, is by definition radiolucent. Most organs and hydrogels are therefore not visible by X-ray fluoroscopic imaging. Polymers 
consist primarily of the lower elements $\mathrm{C}, \mathrm{H}, \mathrm{O}$ and $\mathrm{N}$, so they have very little $\mathrm{X}$ ray absorption. Polymeric biomaterials therefore often require the addition of contrast agents. Medical materials that are required to be radiopaque, either contain metals or inorganic contrast agents (e.g. barium sulphate, zirconium dioxide). The best known example is acrylic bone cement, used for the fixation of total joint replacements, which is mostly made radiopaque by the addition of barium sulphate. However, the addition of contrast agents leads to an inhomogeneous structure, introducing mechanical weakness in the material [75, 83]. Furthermore, inorganic contrast agents in bone cements are associated with bone resorption [84, 85], which is particularly undesirable in osteoporotic persons. Also for a hydrogel nucleus replacement, the addition of a powder, like barium sulphate, is not an option, since it can leach out once the gel is swollen. A disadvantage of the implementation of metallic markers is its incompatibility with MRI, leading to artefacts in the image. Since imaging techniques, like computed tomography (CT) and MRI, are increasingly important, an implant ideally is compatible with both modalities.

To make polymeric biomaterials radiopaque, without the drawbacks of contrast agents and metallic markers, it is possible to introduce a high atomic number halogen into the polymer. For this purpose, a methacrylic monomer was synthesized, containing covalently bound iodine (atomic number $=53$ ) [86]. This monomer, 2-(4'-iodobenzoyl)-oxo-ethyl methacrylate (4IEMA; Figure 14), is readily copolymerized with other methacrylates to yield random copolymers. This way, X-ray absorption is incorporated in the polymer, rendering it intrinsically radiopaque and compatible with both CT and MRI. Moreover, making an implant material intrinsically radiopaque allows complete visualization of the implant, this in contrast to the addition of metallic markers. The stability of these iodine-containing polymers was demonstrated in vivo [87].

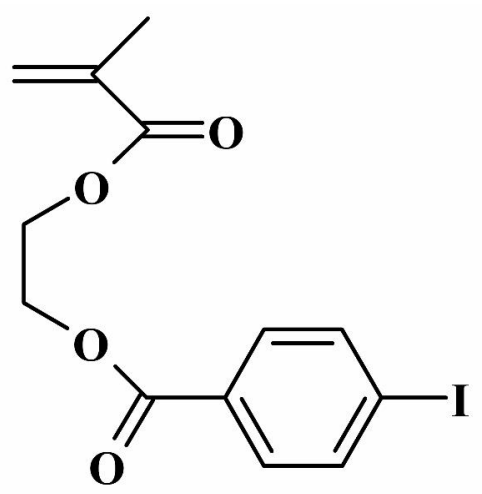

Figure 14: Structural formula of 4IEMA. 
In this work, iodine-containing polymers were used to develop (1) intrinsically radiopaque hydrogels for nucleus pulposus replacement by combining 4IEMA with hydrophilic monomers and (2) a highly radiopaque acrylic bone cement for use in vertebroplasty or kyphoplasty, by replacing the inorganic contrast agent with iodine-containing polymer microspheres.

\section{Outline and aim of this thesis}

With this thesis I hope to contribute to the field of spinal surgery and provide surgeons with viable alternatives in the management of back pain. It describes the attempt to design a new kind of prosthesis for the nucleus pulposus and the development of an all-polymeric bone cement for the augmentation of vertebral compression fractures.

Since nucleus replacement is an emerging technology, a lot of research in this area is done at the moment, resulting in several designs, all building on the pioneering work of the PDN. In our view, most designs have two major flaws, which we wanted to address; nucleus replacements feature insufficient radiopacity and their shape is not in conformity with the nucleus cavity. To achieve a hydrogel implant with intrinsic radiopacity, we combined existing knowledge in our lab from two main research themes, being hydrogel coatings and intrinsically radiopaque polymers. Chapter $\mathbf{2}$ describes the initial investigation of copolymerizing a hydrophobic radiopaque monomer with hydrophilic monomers, attempting to create radiopaque hydrogels. Several properties of the resulting materials, important for the intended application, were investigated. This included water content, stiffness, cytotoxicity and radiopacity. The work resulted in the selection of two materials that seemed promising to serve as nucleus replacements. These two materials were further investigated for their implantability, biocompatibility and radiopacity in situ, as described in Chapter 3. Recognizing that the materials had to be fine-tuned further in order to serve as functional nucleus replacements, the composition was slightly altered and a chemical crosslinker was introduced. Chapter $\mathbf{4}$ then deals with the comparison of the modified and crosslinked materials versus the earlier two materials, and their evaluation on several mechanical properties, important for a functional nucleus prosthesis.

Also, we developed a highly radiopaque iodine-containing bone cement for use in either vertebroplasty or kyphoplasty. This new cement was evaluated for a 
wide array of properties, relevant for the intended application, and is described in Chapter 5.

In Chapter 6 we discuss the implications of both the new nucleus prosthesis and the new cement for the field of spinal surgery and the management of back pain. We will also devise recommendations for future perfection of both appliances.

\section{References}

1. Eidelson SG. Back Pain - A Universal Language. www.spineuniverse.com

2. Koes BW, van Tulder MW, Thomas S. Diagnosis and treatment of low back pain. Bmj 2006; 332: 1430-1434.

3. Nationaal Kompas Volksgezondheid. www.rivm.nl

4. $\quad$ Andersson GB. Epidemiological features of chronic low-back pain. Lancet 1999; 354: 581-585.

5. van Tulder MW, Koes BW, Bouter LM. A cost-of-illness study of back pain in The Netherlands. Pain 1995; 62: 233-240.

6. Druss BG, Marcus SC, Olfson M, Pincus HA. The most expensive medical conditions in America. Health Aff (Millwood) 2002; 21: 105-111.

7. Centraal Bureau voor de Statistiek. www.cbs.nl

8. The Bone and Joint Decade. www.boneandjointdecade.org

9. Nachemson AL. Newest knowledge of low back pain. A critical look. Clin Orthop Relat Res 1992: 8-20.

10. Nachemson AL, Jonsson E (eds.). Neck and Back Pain. Philadelphia: Lippincott Williams \& Wilkins; 2000.

11. White AA, Panjabi MM. Clinical biomechanics of the spine. Philadelphia: Lippincott; 1978.

12. Ihlebaek C, Eriksen HR. The "myths" of low back pain: status quo in norwegian general practitioners and physiotherapists. Spine 2004; 29: 1818-1822.

13. Melton LJ, 3rd. Epidemiology of spinal osteoporosis. Spine 1997; 22: 2S-11S.

14. Melton $\mathrm{D}, 3$ rd, Kallmes DF. Epidemiology of vertebral fractures: implications for vertebral augmentation. Acad Radiol 2006; 13: 538-545.

15. Wu SS, Lachmann E, Nagler W. Current medical, rehabilitation, and surgical management of vertebral compression fractures. J Womens Health (Larchmt) 2003; 12: 17-26.

16. Old JL, Calvert M. Vertebral compression fractures in the elderly. Am Fam Physician 2004; 69: 111-116.

17. Ghosh P (ed.). The biology of the intervertebral disc. vol. 1. CRC Press; 1988.

18. Bogduk N, Twomey LT. Clinical Anatomy of the Lumbar Spine. Edinburgh: Churchill Livingstone; 1987. 
19. Ghosh P (ed.). The biology of the intervertebral disc. vol. 2. CRC Press; 1988.

20. Urban JP, Smith S, Fairbank JC. Nutrition of the intervertebral disc. Spine 2004; 29: 2700-2709.

21. Urban JP, Holm S, Maroudas A, Nachemson A. Nutrition of the intervertebral disk. An in vivo study of solute transport. Clin Orthop Relat Res 1977: 101-114.

22. Urban JP, Holm S, Maroudas A, Nachemson A. Nutrition of the intervertebral disc: effect of fluid flow on solute transport. Clin Orthop Relat Res 1982: 296-302.

23. Urban JP, McMullin JF. Swelling pressure of the lumbar intervertebral discs: influence of age, spinal level, composition, and degeneration. Spine 1988; 13: 179-187.

24. Roberts N, Hogg D, Whitehouse GH, Dangerfield P. Quantitative analysis of diurnal variation in volume and water content of lumbar intervertebral discs. Clin Anat 1998; 11: 1-8.

25. Patel U, Skingle S, Campbell GA, Crisp AJ, Boyle IT. Clinical profile of acute vertebral compression fractures in osteoporosis. $\mathrm{Br}$ J Rheumatol 1991; 30: 418421.

26. Faas A, Chavannes AW, Koes BW, Hoogen JMMvd, Mens JMA, Smeele IJM, Romeijnders ACM, Laan Jvd. Lage-Rugpijn. NHG-standaard. Huisarts en Wetenschap 1996; 39: 18-31.

27. Neurochirurgisch Centrum Nijmegen. http://www.nccn.nl/nccn/

28. Cherkin DC, Deyo RA, Loeser JD, Bush T, Waddell G. An international comparison of back surgery rates. Spine 1994; 19: 1201-1206.

29. Goel VK, Nishiyama K, Weinstein JN, Liu YK. Mechanical properties of lumbar spinal motion segments as affected by partial disc removal. Spine 1986; 11 : 1008-1012.

30. Hanley EN, Jr., Shapiro DE. The development of low-back pain after excision of a lumbar disc. J Bone Joint Surg Am 1989; 71: 719-721.

31. Krismer M. Fusion of the lumbar spine. A consideration of the indications. J Bone Joint Surg Br 2002; 84: 783-794.

32. Ghiselli G, Wang JC, Bhatia NN, Hsu WK, Dawson EG. Adjacent segment degeneration in the lumbar spine. J Bone Joint Surg Am 2004; 86-A: 1497-1503.

33. Gillet $P$. The fate of the adjacent motion segments after lumbar fusion. J Spinal Disord Tech 2003; 16: 338-345.

34. Hilibrand AS, Robbins M. Adjacent segment degeneration and adjacent segment disease: the consequences of spinal fusion? Spine J 2004; 4: 190S-194S.

35. Phillips FM, Reuben J, Wetzel FT. Intervertebral disc degeneration adjacent to a lumbar fusion. An experimental rabbit model. J Bone Joint Surg Br 2002; 84: 289294.

36. Freeman BJ, Davenport J. Total disc replacement in the lumbar spine: a systematic review of the literature. Eur Spine J 2006; 15: 439-447.

37. Guyer RD, Ohnmeiss DD. Intervertebral disc prostheses. Spine 2003; 28: S15-23.

38. Szpalski M, Gunzburg R, Mayer M. Spine arthroplasty: a historical review. Eur Spine J 2002; 11 Suppl 2: S65-84. 
39. Fernstrom U. Arthroplasty with intercorporal endoprothesis in herniated disc and in painful disc. Acta Chir Scand Suppl 1966; 357: 154-159.

40. Ambrosio L, De Santis R, Nicolais L. Composite hydrogels for implants. Proc Inst Mech Eng [H] 1998; 212: 93-99.

41. Kotani Y, Abumi K, Shikinami Y, Takada T, Kadoya K, Shimamoto N, Ito M, Kadosawa T, Fujinaga T, Kaneda K. Artificial intervertebral disc replacement using bioactive three-dimensional fabric: design, development, and preliminary animal study. Spine 2002; 27: 929-935; discussion 935-926.

42. Griffith SL, Shelokov AP, Buttner-Janz K, LeMaire JP, Zeegers WS. A multicenter retrospective study of the clinical results of the LINK SB Charite intervertebral prosthesis. The initial European experience. Spine 1994; 19: 1842-1849.

43. van Ooij A, Oner FC, Verbout AJ. Complications of artificial disc replacement: a report of 27 patients with the SB Charite disc. J Spinal Disord Tech 2003; 16: 369-383.

44. Anderson PA, Rouleau JP. Intervertebral disc arthroplasty. Spine 2004; 29: 27792786.

45. Jacobs JJ, Hallab NJ, Urban RM, Wimmer MA. Wear particles. J Bone Joint Surg Am 2006; 88 Suppl 2: 99-102.

46. Herkowitz HN. Total disc replacement with the CHARITE artificial disc was as effective as lumbar interbody fusion. J Bone Joint Surg Am 2006; 88: 1168.

47. Kulkarni AG, Diwan AD. Prosthetic lumbar disc replacement for degenerative disc disease. Neurol India 2005; 53: 499-505.

48. Bao QB, Yuan HA. New technologies in spine: nucleus replacement. Spine 2002; 27: $1245-1247$.

49. Goins ML, Wimberley DW, Yuan PS, Fitzhenry LN, Vaccaro AR. Nucleus pulposus replacement: an emerging technology. Spine J 2005; 5: 317S-324S.

50. Mochida J. New strategies for disc repair: novel preclinical trials. J Orthop Sci 2005; 10: 112-118.

51. Carl A, Ledet $E$, Yuan $H$, Sharan A. New developments in nucleus pulposus replacement technology. Spine J 2004; 4: 325S-329S.

52. Korge A, Nydegger T, Polard JL, Mayer HM, Husson JL. A spiral implant as nucleus prosthesis in the lumbar spine. Eur Spine J 2002; 11 Suppl 2: S149-153.

53. Boyd LM, Carter AJ. Injectable biomaterials and vertebral endplate treatment for repair and regeneration of the intervertebral disc. Eur Spine J 2006; 15 Suppl 15: 414-421.

54. Di Martino A, Vaccaro AR, Lee JY, Denaro V, Lim MR. Nucleus pulposus replacement: basic science and indications for clinical use. Spine 2005; 30: S1622.

55. Shim CS, Lee SH, Park CW, Choi WC, Choi G, Choi WG, Lim SR, Lee HY. Partial disc replacement with the PDN prosthetic disc nucleus device: early clinical results. J Spinal Disord Tech 2003; 16: 324-330.

56. Klara PM, Ray CD. Artificial nucleus replacement: clinical experience. Spine 2002; 27: $1374-1377$. 
57. Gamradt SC, Wang JC. Lumbar disc arthroplasty. Spine J 2005; 5: 95-103.

58. Roughley P, Hoemann C, DesRosiers E, Mwale F, Antoniou J, Alini M. The potential of chitosan-based gels containing intervertebral disc cells for nucleus pulposus supplementation. Biomaterials 2006; 27: 388-396.

59. Anderson DG, Risbud MV, Shapiro IM, Vaccaro AR, Albert TJ. Cell-based therapy for disc repair. Spine J 2005; 5: 297S-303S.

60. Acosta FL, Jr., Lotz J, Ames CP. The potential role of mesenchymal stem cell therapy for intervertebral disc degeneration: a critical overview. Neurosurg Focus 2005; 19: E4.

61. Lotz JC, Kim AJ. Disc regeneration: why, when, and how. Neurosurg Clin N Am 2005; 16: 657-663, vii.

62. Horner HA, Urban JP. 2001 Volvo Award Winner in Basic Science Studies: Effect of nutrient supply on the viability of cells from the nucleus pulposus of the intervertebral disc. Spine 2001; 26: 2543-2549.

63. Garfin SR, Yuan HA, Reiley MA. New technologies in spine: kyphoplasty and vertebroplasty for the treatment of painful osteoporotic compression fractures. Spine 2001; 26: 1511-1515.

64. Deramond H, Depriester C, Galibert P, Le Gars D. Percutaneous vertebroplasty with polymethylmethacrylate. Technique, indications, and results. Radiol Clin North Am 1998; 36: 533-546.

65. Roermund PMv, Plasmans CMT, Donk R, Oner FC, Kleuver Md, Ooij Av, Verbout AJ. Orthopedisch wondermiddel. Medisch Contact 2002; 57: 670.

66. Oner FC, de Kleuver M, van Ooy A, Verbout AJ. [The disc prosthesis: myths and facts]. Ned Tijdschr Geneeskd 2002; 146: 1625-1631.

67. van Hooy-Corstjens CSJ, Govaert LE, Spoelstra AB, Bulstra SK, Wetzels GM, Koole $\mathrm{LH}$. Mechanical behaviour of a new acrylic radiopaque iodine-containing bone cement. Biomaterials 2004; 25: 2657-2667.

68. Peerlings CC, Hanssen HH, Bevers RT, Boelen EJH, Stelt BJ, Korthagen EJ, Koole LH. Heparin release from slippery-when-wet guide wires for intravascular use. J Biomed Mater Res 2002; 63: 692-698.

69. Bao QB, Yuan HA. Prosthetic disc replacement: the future? Clin Orthop 2002: 139-145.

70. Sieber AN, Kostuik JP. Concepts in nuclear replacement. Spine J 2004; 4: 322S$324 S$.

71. Ray CD. The PDN prosthetic disc-nucleus device. Eur Spine J 2002; 11 Suppl 2: S137-142.

72. Bertagnoli R, Vazquez RJ. The Anterolateral TransPsoatic Approach (ALPA): a new technique for implanting prosthetic disc-nucleus devices. J Spinal Disord Tech 2003; 16: 398-404.

73. Jin D, Qu D, Zhao L, Chen J, Jiang J. Prosthetic disc nucleus (PDN) replacement for lumbar disc herniation: preliminary report with six months' follow-up. J Spinal Disord Tech 2003; 16: 331-337. 
74. Kühn K-D. Bone cements. Up-to-date comparison of physical and chemical properties of commercial materials. Berlin: Springer-Verlach; 2000.

75. Bhambri SK, Gilbertson LN. Micromechanisms of fatigue crack initiation and propagation in bone cements. J Biomed Mater Res 1995; 29: 233-237.

76. Mathis JM, Barr JD, Belkoff SM, Barr MS, Jensen ME, Deramond H. Percutaneous vertebroplasty: a developing standard of care for vertebral compression fractures. AJNR Am J Neuroradiol 2001; 22: 373-381.

77. Provenzano MJ, Murphy KP, Riley $\mathrm{LH}$, 3rd. Bone cements: review of their physiochemical and biochemical properties in percutaneous vertebroplasty. AJNR Am J Neuroradiol 2004; 25: 1286-1290.

78. Gangi A, Guth S, Imbert JP, Marin H, Dietemann JL. Percutaneous vertebroplasty: indications, technique, and results. Radiographics 2003; 23: e10.

79. Belkoff SM, Sanders JC, Jasper LE. The effect of the monomer-to-powder ratio on the material properties of acrylic bone cement. J Biomed Mater Res 2002; 63: 396-399.

80. Jasper LE, Deramond $\mathrm{H}$, Mathis JM, Belkoff SM. Material properties of various cements for use with vertebroplasty. J Mater Sci Mater Med 2002; 13: 1-5.

81. Link DP, Mourtada FA, Jackson J, Blashka K, Samphilipo MA. Hydrogel embolic agents. Theory and practice of adding radio-opacity. Invest Radiol 1994; 29: 746751.

82. Hubbell $\mathrm{JH}$. Review of photon interaction cross section data in the medical and biological context. Phys Med Biol 1999; 44: R1-22.

83. Ginebra MP, Albuixech L, Fernandez-Barragan E, Aparicio C, Gil FJ, San RJ, Vazquez B, Planell JA. Mechanical performance of acrylic bone cements containing different radiopacifying agents. Biomaterials 2002; 23: 1873-1882.

84. Sabokbar A, Fujikawa Y, Murray DW, Athanasou NA. Radio-opaque agents in bone cement increase bone resorption. J Bone Joint Surg Br 1997; 79: 129-134.

85. Ingham E, Green TR, Stone MH, Kowalski R, Watkins N, Fisher J. Production of TNF-alpha and bone resorbing activity by macrophages in response to different types of bone cement particles. Biomaterials 2000; 21: 1005-1013.

86. Benzina A, Kruft MAB, Bar F, van der Veen FH, Bastiaansen CW, Heijnen V, Reutelingsperger $\mathrm{C}$, Koole $\mathrm{LH}$. Studies on a new radiopaque polymeric biomaterial. Biomaterials 1994; 15: 1122-1128.

87. Aldenhoff YB, Kruft MAB, Pijpers AP, van der Veen FH, Bulstra SK, Kuijer R, Koole LH. Stability of radiopaque iodine-containing biomaterials. Biomaterials 2002; 23: 881-886. 


\section{Intrinsically Radiopaque Hydrogels for Nucleus Pulposus Replacement}

Erik J.H. Boelen, Catharina S.J. van Hooy-Corstjens, Sjoerd K. Bulstra, André van Ooij, Lodewijk W. van Rhijn and Leo H. Koole

Adapted from:

Biomaterials 26 (2005) 6674-6683

The work described in this paper also led to the filing of a patent:

Radiopaque Prosthetic Intervertebral Disc Nucleus WO2006/028370

(Appendix 3). 


\section{Abstract}

Degeneration of the intervertebral disc is the most common cause of back pain. In case of early stage degenerative disc disease or traumatic herniations, a suitable treatment may be to replace the nucleus pulposus, preserving the annulus fibrosus. Eight new hydrogel biomaterials were prepared and studied for their potential as a nucleus replacement. The hydrogels were designed according to the following criteria: (i), they should exhibit adequate radiopacity; (ii), they should be non-cytotoxic; (iii), implantation in the dry state and subsequent swelling in situ to fill the entire nucleus cavity; (iv), after swelling they should match the physical-mechanical properties of the native nucleus. The approach was to use copolymers consisting of 2-(4'-iodobenzoyl)-oxo-ethyl methacrylate (4IEMA) and a hydrophilic building block (either $\mathrm{N}$-vinyl-2pyrrolidinone (NVP) or 2-hydroxyethyl methacrylate (HEMA)); 4 copolymers of NVP/4IEMA and 4 copolymers of HEMA/4IEMA in different compositions $(5,10$, 15 and 20 mol\% 4IEMA).

The study comprised ${ }^{1} \mathrm{H}$-NMR analysis of the copolymerization reaction NVP + 4IEMA. Furthermore, the copolymers were studied with respect to their swelling behaviour, mechanical properties, cytotoxicity in vitro and X-ray contrast. Hydrogels with 5 mol\% 4IEMA appear to meet all criteria: they are noncytotoxic, have adequate physical-mechanical properties and feature sufficient radiopacity in a realistic model. The potential implications of these new results with respect to treatment of degenerative disc disease are discussed briefly. 


\section{Introduction}

Back pain is the largest cause of lost work days and activity limitation in western countries for people younger than 45 years [1, 2]. The most common cause is degeneration of the intervertebral discs (IVDs) [3]. Various conservative treatment options are available. When these fail to alleviate the pain, surgical intervention is often inevitable. Two common operations are: (i) removal of the damaged IVD(s) (discectomy) and/or spinal fusion, or (ii) replacement of the IVD with a prosthetic disc [4]. The first option is primarily concerned with alleviating pain, whereas the latter is also focused on restoring function. The structural and functional complexity of the IVD makes it hard to match Nature's design. There is a wide variety of prosthetic discs [5], but only few have made it to clinical use and even less were somewhat successful.

In some cases, back pain originates from a traumatic rupture of the annulus fibrosus, in such a way that the defect allows herniation of the nucleus pulposus. Then, adequate treatment may be to remove the herniated nucleus and replace it with a prosthetic implant, followed by closure of the annulus defect e.g. via a tissue engineering strategy. This treatment can also be suitable to help patients with early-stage disc degeneration. Replacing only the nucleus has several advantages over (i) and (ii) as it is less invasive, and the remaining disc tissues, i.e. the annulus and the endplates, are preserved, as well as their functions $[3,6]$. The height and mobility of the IVD are maintained and overloading of the adjacent levels, which is often a result of spinal fusion, is prevented.

The natural nucleus pulposus is a gelly substance. Its water content is $>80 \%$ in children, and is shown to decrease gradually with age [7]. Its main function, as part of the IVD, is to provide flexibility to the spine. The material of choice to replace the damaged nucleus pulposus would be a synthetic hydrogel. Numerous synthetic hydrogels are known, most of them have a high level of biocompatibility, and their physical-mechanical properties can be tuned to mimic the behaviour of the natural nucleus $[8,9]$. A prominent example is the Prosthetic Disc Nucleus (PDN), developed by Raymedica Inc. [10-14]. One of the shortcomings of the PDN may be that it does not completely fill the cavity left by the removed nucleus. Filling the cavity entirely is essential to achieve a physiological stress distribution within the disc $[15,16]$ and to minimize implant migration. 
The success of a nucleus replacement is also largely dependent on the correct positioning of the implant. To assess adequately the location of the implant during and after surgery, it is convenient that it can be monitored real-time using X-ray fluoroscopy. That way the surgeon is able to perfectly position the implant in the nucleus cavity. Also, in case of complications arising at a later time, the implant can be easily traced.

Herein, we report initial studies on new hydrogel biomaterials with potential utility as a nucleus prosthesis. For this, four criteria were defined: (1) The hydrogel prosthesis should exhibit intrinsic radiopacity; (2) The hydrogel prosthesis should be non-cytotoxic; (3) The prosthesis, being implanted in dry form (which is relatively small) should swell in situ to fill the entire cavity that is left after complete removal of the nucleus; (4) In the swollen state, the physical-mechanical properties of the prosthesis should match those of the native nucleus as closely as possible.

Our strategy to meet these criteria was based on the use of copolymers, which are synthesized from a hydrophilic building block (either N-vinyl-2-pyrrolidinone (NVP) or 2-hydroxyethyl methacrylate (HEMA)) and a hydrophobic iodinecontaining building block, i.e. 2-(4'-iodobenzoyl)-oxo-ethyl methacrylate (4IEMA; Figure 1) [17-20]. The iodine in the 4IEMA is covalently bound and renders the copolymers intrinsically radiopaque, while the ratio hydrophilic : hydrophobic determines important characteristics such as swelling behaviour and stiffness.

Eight different hydrogels were prepared, characterized and studied with respect to their biocompatibility, X-ray contrast and physical-mechanical properties, while keeping in mind the suitability for use as nucleus pulposus substitute.

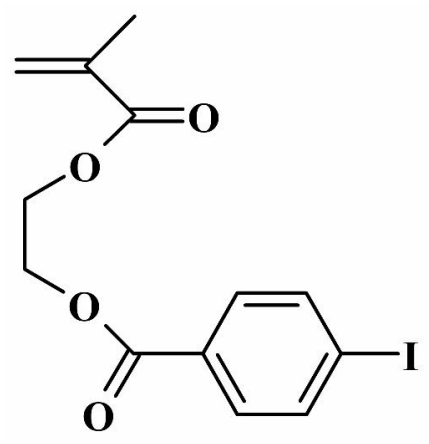

Figure 1: Structural formula of 4IEMA. 


\section{Materials and methods}

\subsection{Materials}

All chemicals were purchased from Acros (Landsmeer, The Netherlands). NVP and HEMA were distilled under reduced pressure to remove inhibiting additives. The monomer 4IEMA was synthesized from 4-iodobenzoyl chloride and purified HEMA [17]. Purity and identity of all monomers was checked by ${ }^{1} \mathrm{H}-\mathrm{NMR}$ spectroscopy. 2,2'-azobis(isobutyronitrile) (AIBN) was used as the source of free radicals. Monomers and $0.044 \mathrm{~mol} \%$ of AIBN were mixed and transferred to Teflon tubes with an inner diameter of $12 \mathrm{~mm}$, which were closed with a stopper on one end. Then they were immersed in a thermostated oil bath. A temperature profile was run that keeps the oil bath for 8 hours at $60{ }^{\circ} \mathrm{C}$, followed by 4 hours at $80^{\circ} \mathrm{C}$ and another 4 hours at $100^{\circ} \mathrm{C}$.

Polymerization resulted in transparent, glassy rods. Eight different copolymers were prepared and their composition will be indicated in codes: a letter followed by a number indicating respectively the hydrophilic monomer (NVP or HEMA) and its molar percentage; e.g. N95 is a copolymer of $95 \mathrm{~mol} \% \mathrm{NVP}$ and 5 mol\% 4IEMA. The prepared copolymers are N95, N90, N85, N80, H95, H90, $\mathrm{H} 85$ and $\mathrm{H} 80$.

\subsection{Copolymerization of NVP and 4IEMA}

The reaction of NVP + 4IEMA was studied according to the method of Kruft et al. [21]. Monomer mixtures of NVP and 4IEMA (0.09, 0.14, 0.18, 0.32, 0.43, 0.52 molar fraction 4IEMA) and $0.1 \mathrm{~mol} \%$ AIBN were dissolved in DMSO- $\mathrm{d}_{6}(1.5$ $\mathrm{mmol} / \mathrm{mL}$ ). About $700 \mu \mathrm{L}$ was transferred into a $5 \mathrm{~mm}$ diameter NMR tube. A ${ }^{1} \mathrm{H}-\mathrm{NMR}$ spectrum (Varian Unity-Plus, $400 \mathrm{MHz}$ ) was taken at room temperature to check purity and composition of the monomer mixture. Subsequently the sample was rapidly heated to $70{ }^{\circ} \mathrm{C}$ inside the magnet. ${ }^{1} \mathrm{H}-\mathrm{NMR}$ spectra were taken every 3 minutes to follow the polymerization reaction.

The glass transition temperature $\left(T_{g}\right)$ of the materials in the dry state was measured on a Perkin Elmer Pyris 1 DSC at a heating rate of $10 \mathrm{~K} / \mathrm{min}$. 


\subsection{Swelling}

To study the water uptake of the different materials, swelling experiments in phosphate buffered saline (PBS) were conducted in triplo, using sample discs with a diameter of $12 \mathrm{~mm}$ and a thickness of $2 \mathrm{~mm}$. Material masses and dimensions of the discs were measured at different time intervals. The excess of water from the surface was removed before the samples were weighed. After weighing, the samples were put back in fresh, ample PBS. The water content (WC) was calculated using equation 1.

$$
W C=100 \times \frac{m_{s}-m_{d}}{m_{s}}
$$

$m_{s}$ is the mass of the swollen sample and $m_{d}$ is the mass of the dry sample. Also the swelling ratio was determined, which is defined by the increase in volume: the volume of the swollen sample in equilibrium, divided by the volume of the dry sample.

\subsection{Cytotoxicity}

The cytotoxicity of the materials was evaluated. Therefore, fragments of the samples were UV-sterilized and incubated in medium for 3 days at $37{ }^{\circ} \mathrm{C}$. The medium, with the extracts of the samples, was put on 3T3 mouse fibroblasts and cell viability (the cell survival in pure medium was set to $100 \%$ ) was checked after 4 days of culturing $\left(37{ }^{\circ} \mathrm{C}, 5 \% \mathrm{CO}_{2}\right.$ ) using the MTT assay [22, 23]. Direct-contact tests were performed with $3 \mathrm{~T} 3$ mouse fibroblasts on the materials surface of $1 \mathrm{~cm}$ diameter discs. Glass slides were used as a reference. The amount of cells on the materials surface was approximately $5 \times 10^{5}$ cells $/ \mathrm{cm}^{2}$. The Live/Dead assay (Molecular Probes, Reduced Biohazard Viability/Cytotoxicity Kit \#1 (L-7013)) was used to evaluate adhering cells after $24 \mathrm{~h}$ by fluorescence microscopy (Nikon Eclipse E800 equipped with a Coolsnap camera (RS Photometric, USA)) [24].

\subsection{X-Ray opacity}

X-ray visibility was checked by taking an X-ray photograph (40 kV, $4 \mathrm{mAs}$ ) of the swollen discs, used in the swelling experiments. As a pilot study, dry oval samples (2.5 mm thick) of $\mathrm{H} 95$ and $\mathrm{H} 90$ were inserted into a nucleus cavity of 
the lumbar spine of a porcine cadaver by a certified orthopedic surgeon and after overnight swelling X-ray photographs were taken of the materials in situ at clinical conditions (66 kV, automatic exposure). This provides a realistic model.

\subsection{Static mechanics}

To determine the Young's modulus of the swollen hydrogels, a static compression experiment was performed in which swollen samples of ca. $8 \mathrm{~mm}$ thickness and ca. $15 \mathrm{~mm}$ diameter were used. Three samples of each material were, at room temperature, loaded to about $80 \%$ compression at a true strain rate of $3 \times 10^{-3} \mathrm{~s}^{-1}$, on a MTS 810 servo-hydraulic tension-compression tester using a $25 \mathrm{kN}$ load cell. Compression was stopped as soon as cracks were observed.

\subsection{Rheology}

To assess the rheological properties of the designed hydrogels, rheological experiments were performed on swollen discs (diameter $8-16 \mathrm{~mm}$, thickness $1.2 \mathrm{~mm}$ ) using a parallel plate viscometer (ARES 3LSLC1, Rheometric Scientific) at room temperature. The samples were compressed to a normal force of around $0.5 \mathrm{~N}$. First a strain sweep $(0.005 \%-0.5 \%)$ was performed at an angular frequency of $1 \mathrm{rad} / \mathrm{s}$ on samples with a high (N80) and low (N95) modulus to determine the region of linear viscoelasticity. From this experiment it was conducted that a strain of $0.01 \%$ was in the linear viscoelastic region of both materials. Therefore, the dynamic frequency experiments of all samples were performed at this strain.

\subsection{Hysteresis}

Cyclic compression experiments were conducted to investigate the elastic behaviour of the hydrogels for physiologic strains. Sample sizes were the same as for the static experiment. It was hypothesized, based on average disc stiffness and minimum disc strength for an artificial disc [25], that a nucleus prosthesis should be able to withstand 30\% compression without plastic deformation. Material samples were compressed to $30 \%$ within 10 seconds and then the tester (MTS 810 ) returned in 10 seconds to the starting position. This 
was repeated 10 times and a duplicate was performed. The materials were not constrained radially.

\section{Results and discussion}

\subsection{Copolymerization of NVP and 4IEMA}

From our previous work on the free radical copolymerization reaction HEMA + 4IEMA, the reactivity ratios are known: $r_{4 \text { IIEMA }}=2.18 \pm 0.72, r_{\text {HEMA }}=2.29 \pm$ 0.38 ) [21]. Both $r$ values are close to unity, which implies that the HEMA/4IEMA copolymers are random-type. NVP is known to be significantly less reactive than methacrylate monomers. For example, the reactivity ratios of the copolymerization of methyl methacrylate (MMA) + NVP are $r_{M M A}=3.95$ and $r_{N V P}$ $=4.3 \times 10^{-3}$ (calculated using $Q$ and $e$ values from Greenley [26]).

We decided to study the free radical copolymerization NVP + 4IEMA in detail, following the ${ }^{1} \mathrm{H}-\mathrm{NMR}$ method as described by Kruft et al [21]. Six reactions were run, each starting with a different feed composition. A series of ${ }^{1} \mathrm{H}-\mathrm{NMR}$ spectra was recorded during each reaction; each acquisition took approximately $20 \mathrm{sec}$.

As a typical example, Figure 2 shows expansions of the ${ }^{1} \mathrm{H}-\mathrm{NMR}$ spectra which were recorded for $f_{4 I E M A}=0.43$ (molar fraction 4IEMA). Figure $2 \mathrm{~A}$ refers to the start of the reaction ( $0 \%$ conversion); Figure $2 \mathrm{~B}$ refers to $15 \%$ conversion. Note the broadened peaks $a_{1}{ }^{\prime}$ and $a_{2}{ }^{\prime}(\delta 7.8 \mathrm{ppm}$ and $\delta 7.7 \mathrm{ppm})$ which correspond to the aromatic protons of built-in 4IEMA. The spectra were analyzed as described in Appendix 2. The reactivity ratios were determined at ca. $5 \%$ conversion (i) and ca. 15\% conversion (ii). Both approaches resulted in comparable values for $r_{\text {AIEMA }}$ and $r_{\text {NVP: }}$ : Method (i): $r_{\text {AIEMA }}=5.4 \pm 0.79$ and $r_{\text {NVP }}=$ $0.04 \pm 0.03$. Method (ii): $r_{4 \text { IEMA }}=4.7 \pm 0.32$ and $r_{N V P}=0.04 \pm 0.006$.

The $r$ values clearly demonstrate that 4IEMA is much more reactive than NVP. Noteworthy, the $r$ values for the reaction of NVP + 4IEMA are in good agreement with the $r$ values calculated using the $Q$ and $e$ values from Greenley [26] for the copolymerization of MMA and NVP $\left(r_{M M A}=3.95\right.$ and $r_{N V P}=4.3 \times$ $\left.10^{-3}\right)$. 


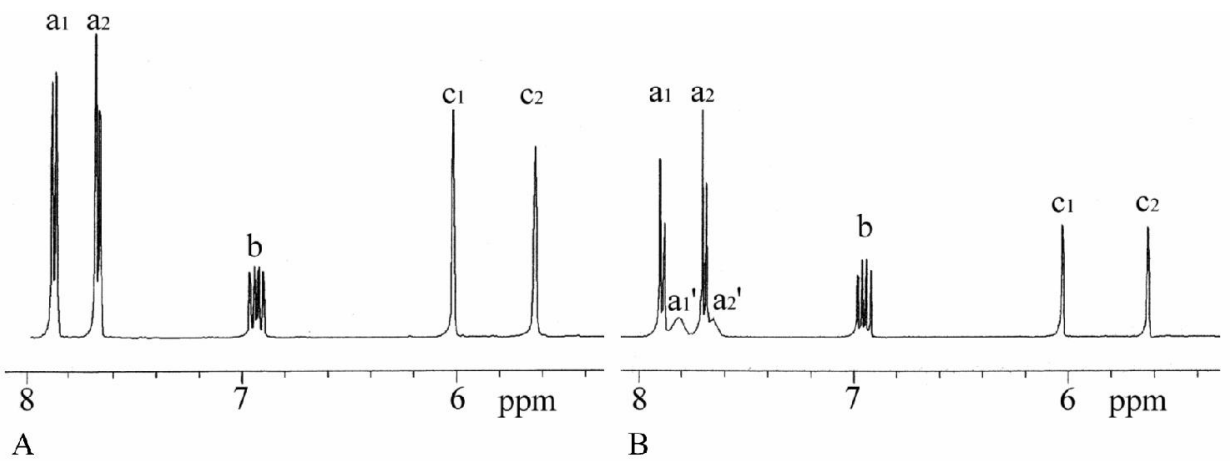

Figure 2: Expansion ( $\delta=5.4-8.0 \mathrm{ppm}$ ) of the $400 \mathrm{MHz}{ }^{1} \mathrm{H}-\mathrm{NMR}$ spectra of monomer mixture NVP/4IEMA (f AIEMA $\left._{1}=0.43\right)$ at $(A) 0 \%$ conversion and $(B) 15 \%$ conversion. Assignments are as follows: $a_{1}$ and $a_{2}$ : aromatic protons of 4IEMA; $a_{1}{ }^{\prime}$ and $a_{2}{ }^{\prime}$ : aromatic protons of built-in 4IEMA; $b$ : vinyl proton of NVP; $c_{1}$ and $c_{2}$ : vinyl protons of 4IEMA.

Figure 3 compiles the data from the analyses. The composition of the copolymer ( $F$ ) is plotted as a function of the monomer feed composition ( $f$ ). The curves from analyses (i) and (ii) deviate substantially from the diagonal, reflecting that 4IEMA is consumed preferentially. Moreover, it is clear that the curves resulting from analyses (i) and (ii) almost coincide.

It can now be concluded that our materials N80, N85, N90 and N95 are not random-type copolymers. It must be assumed that most of the 4IEMA monomer molecules are consumed at low conversion. In other words, at high conversion, the reaction involves incorporation of (predominantly) NVP molecules, resulting in NVP oligomers.

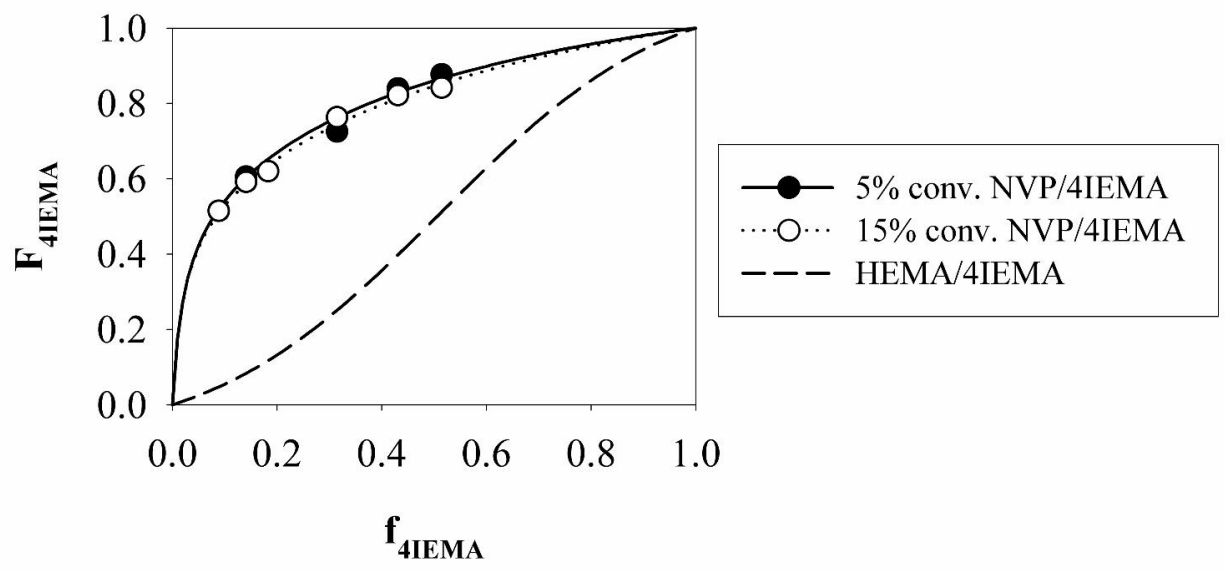

Figure 3: Copolymer composition as a function of monomer feed. 
${ }^{1} \mathrm{H}-\mathrm{NMR}$ spectra of the copolymers NVP/4IEMA, dissolved in DMSO- $\mathrm{d}_{6}$, show the presence of unreacted NVP ( $2 \mathrm{~mol} \%)$, but no unreacted 4IEMA. The presence of residual monomer prompted us to wash each sample in distilled water, prior to all further analyses. After washing the samples, the weight loss was larger than expected on basis of residual monomer dissolution. It was therefore concluded that also NVP oligomers dissolve during washing, which was also seen by others [27]. As a result, the ratios NVP/4IEMA have changed and the new ratios can be calculated, based on the difference in mass between the dried, washed and the original samples. The new molar ratios are: N95: 93/7, N90: 85/15, N85: 77/23, N80: 69/31. The copolymer composition after washing was corroborated by ${ }^{1} \mathrm{H}-\mathrm{NMR}$ analysis. Futher confirmation was derived from DSC experiments. For all compositions, a single $T_{g}$ has been measured, suggesting that a homogeneous system is obtained. For such homogeneous system, where no separate domains of homopolymer are present, the Fox equation [28] can be used to predict the $T_{g}$. Based on a $T_{g}$ of $179^{\circ} \mathrm{C}$ for PVP [29] and $75{ }^{\circ} \mathrm{C}$ for P4IEMA [30], the theoretical glass transition temperatures are predicted and compared to the measured $T_{g}{ }^{\prime} s$ of the washed and subsequently dried copolymers (Table 1 ). The measured values are indeed very comparable to the predicted values, validating the assumption that the weight loss can be fully ascribed to loss of NVP monomers and oligomers. ${ }^{1} \mathrm{H}-\mathrm{NMR}$ spectra of the washed and subsequently dried NVP/4IEMA copolymers show no residual monomer anymore.

Table 1: Glass transition temperatures $\left({ }^{\circ} \mathrm{C}\right)$.

\begin{tabular}{lll}
\hline Code & Measured & Predicted \\
\hline N95 & 155 & 154 \\
N90 & 139 & 135 \\
N85 & 124 & 121 \\
N80 & 116 & 111 \\
\hline
\end{tabular}

Based on the reactivity ratios, the copolymers of HEMA and 4IEMA are assumed to be random-type. The HEMA/4IEMA copolymers appeared to be insoluble in all solvents, probably due to a high molar mass and physical and chemical crosslinking [31-33]. Therefore no ${ }^{1} \mathrm{H}-\mathrm{NMR}$ spectra could be taken. We decided to give the HEMA/4IEMA copolymers exactly the same wash treatment as the NVP/4IEMA copolymers. However, unlike the NVP-based copolymers, no mass loss was observed while washing. 


\subsection{Swelling}

The volume increase and the water uptake of the eight different copolymers were determined by swelling discs of the copolymers in PBS at room temperature. Figure 4 shows the water content vs. time for the 8 different copolymers. The NVP-based hydrogels have a higher water content than the HEMA-based hydrogels, confirming that NVP is more hydrophilic than HEMA. It also follows that the hydrophobic nature of 4IEMA has a drastic effect on the water uptake of the hydrogels; for both types of hydrogel, the water content decreases significantly going from low to high 4IEMA content. Also the rate at which the hydrogels imbibe water depends on the hydrophilic character of the hydrogel. Whereas for the more hydrophilic hydrogels (N95, N90, N85) equilibrium water content (EWC) is reached in about 12 hours, the less hydrophilic hydrogels take 2 days or more to reach equilibrium swelling. The swelling ratios were determined by measuring sample volume (based on thickness and diameter), before and after swelling. Swelling ratios are: N95: 4.5, N90: 2.9, N85: 2.0, N80: 1.4, H95: 1.6, H90: 1.6, H85: 1.3, H80: 1.2. These swelling ratios can be used to calculate the size of the nucleus prosthesis before implantation, based on the dimensions of the nucleus cavity.

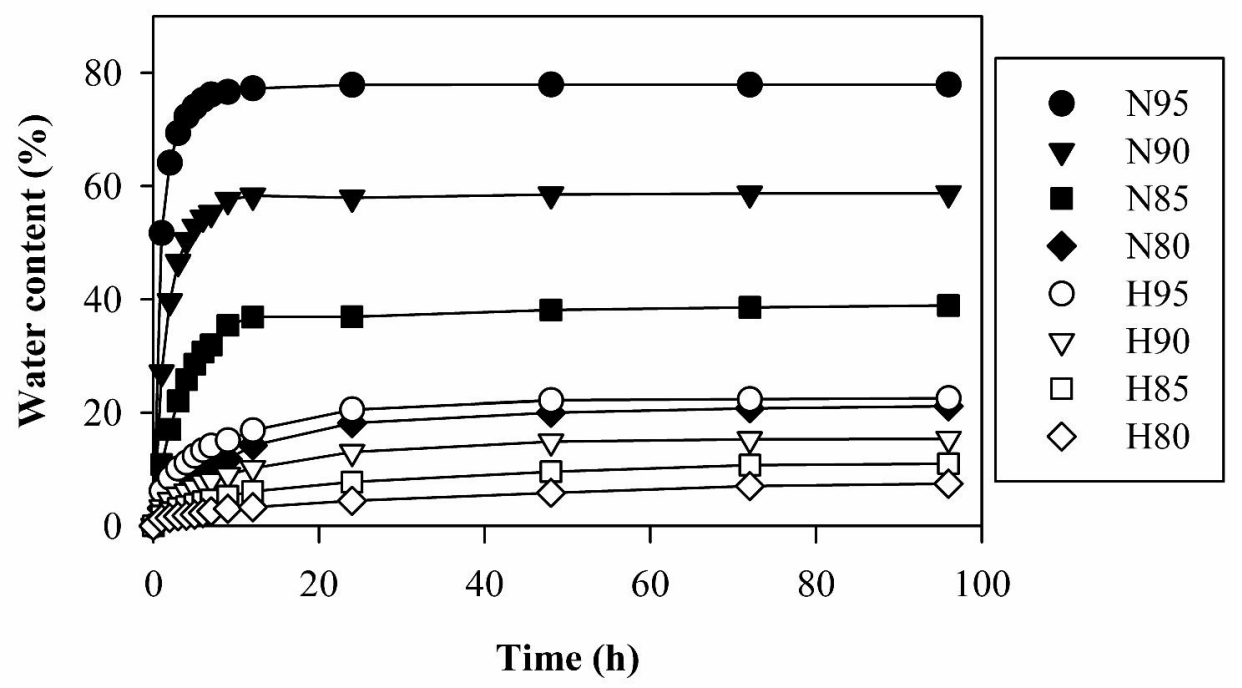

Figure 4: Swelling curves of the copolymers in PBS at room temperature. 


\subsection{Cytotoxicity}

The possible cytotoxicity of our materials (after washing) was assessed through the MTT test and the Live/Dead assay. The results of the MTT tests are displayed in Figure 5. The NVP-based hydrogels are non-cytotoxic, whereas the HEMA-based hydrogels seem to become somewhat cytotoxic upon decreasing their hydrophilicity. Figure 6 shows micrographs that were obtained with the Live/Dead assay. These data were not treated quantitatively. All surfaces contain mostly live (green) cells and only a few dead (red) cells, indicating excellent biocompatibility of the hydrogels in direct contact with cells. It is seen that the cells prefer less hydrophilic surfaces. Cells on the most hydrophilic materials (N95, N90, N85 and H95) tend to form clusters.

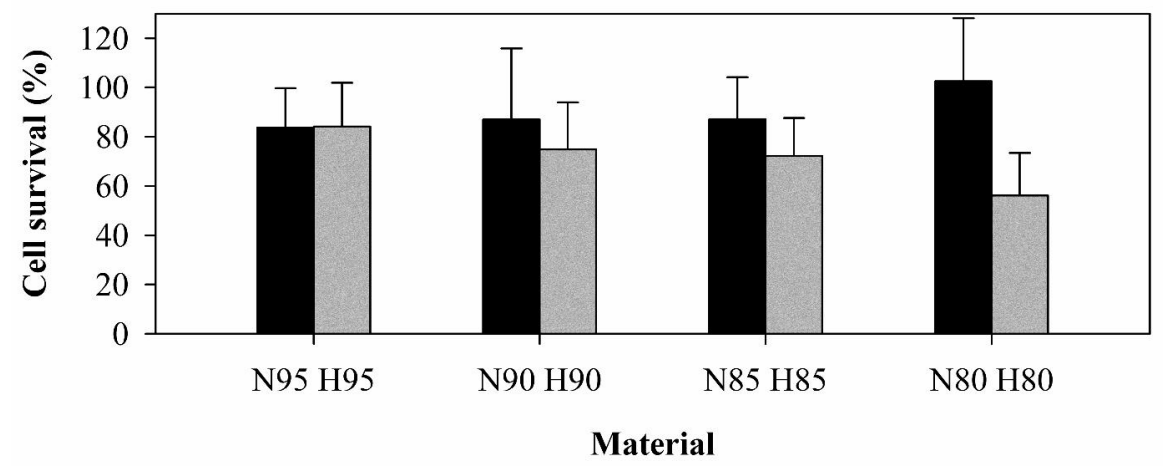

Figure 5: Cell survival of 3T3 mouse fibroblast cells as measured with the MTT assay. Cell survival in medium was set to $100 \%$. 

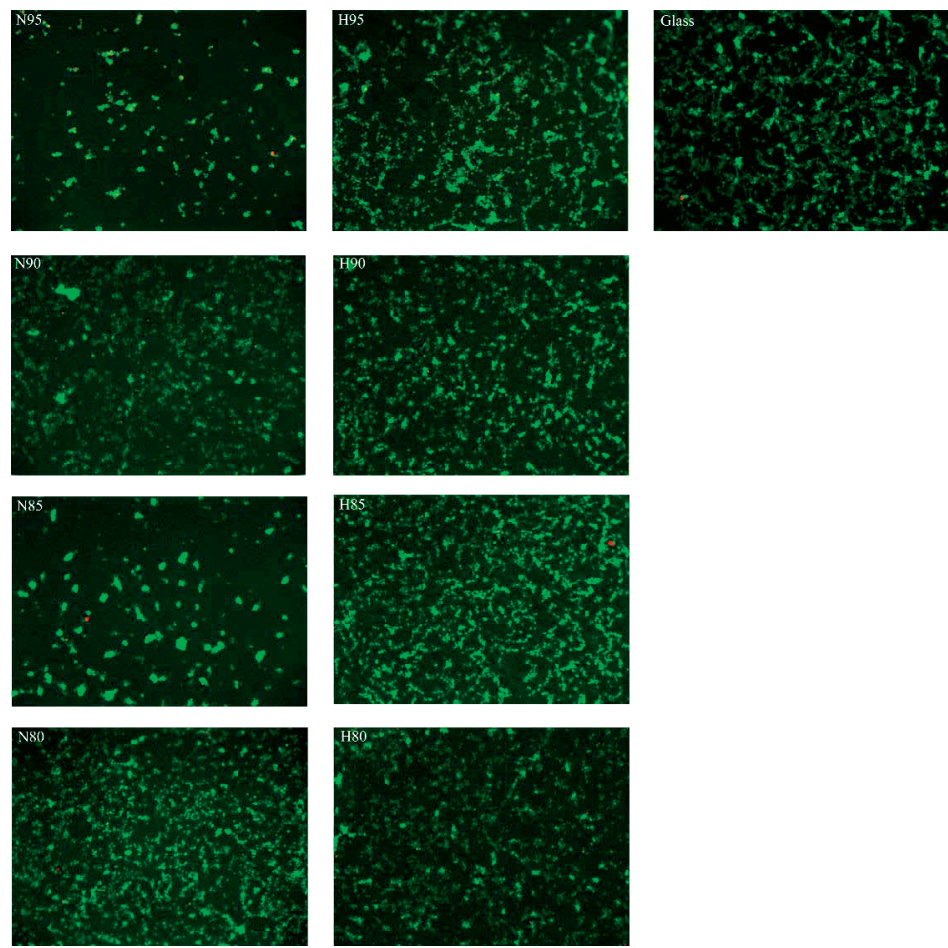

Figure 6: Fluorescent images resulting from the Live/Dead staining assay of $3 T 3$ mouse fibroblast cells in contact with the hydrogels.

\subsection{X-Ray opacity}

Figure 7 shows the X-ray absorption image of the 8 hydrogels in their swollen state. Increasing the iodine content from $\mathrm{H} 95$ to $\mathrm{H} 80$ and from N95 to N80 clearly leads to improved contrast. To answer the question whether the level of contrast of the gels would be sufficient for imaging inside the spinal column, we decided to do a preliminary experiment, in which the materials H95 and H90 were implanted at two adjacent levels between two vertebrae of a porcine cadaver. Figure 8 shows the resulting X-ray image, which was recorded under routine hospital conditions. Three intervertebral structures are seen: the native disc (*) below, gel H90 in the middle and gel H95 on top. The native discus is seen as a non-absorbing (white) band, while $\mathrm{H} 95$ and $\mathrm{H} 90$ are clearly visible as $X$-ray absorbing structures. It can be concluded that 5 mol\% 4IEMA already introduces adequate $\mathrm{X}$-ray visibility. 


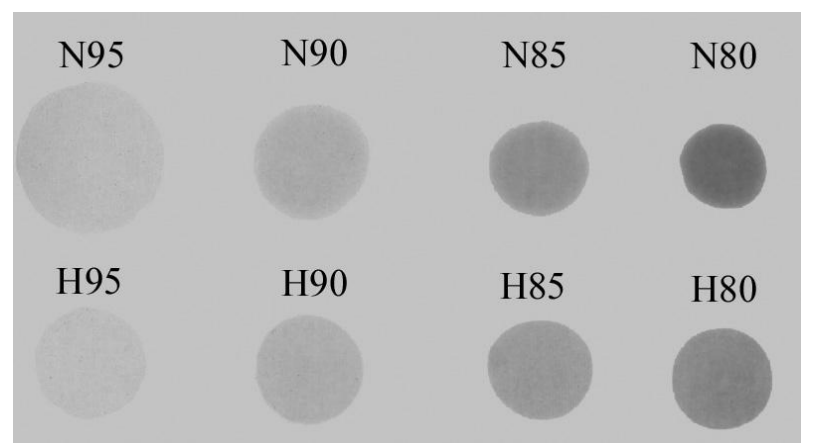

Figure 7: X-ray image of swollen hydrogel discs recorded at $40 \mathrm{kV}$.

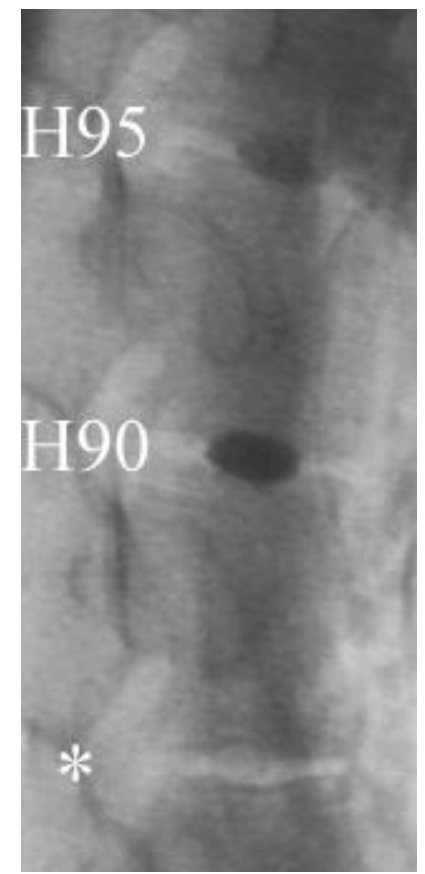

Figure 8: X-ray image of two hydrogels implanted in an IVD of a porcine cadaver, recorded at clinical conditions $(66 \mathrm{kV})$. * indicates an intact intervertebral disc.

\subsection{Static mechanics}

The water in the hydrogels has a plasticizing effect and decreases the stiffness of the copolymers. Compression experiments were performed to determine the Young's moduli of the swollen hydrogels. The results of these compression experiments are depicted in Figure 9. Dehydration effects were assumed to be 
minimal in the time span used for compression. H85 and H80 samples were omitted in this and further experiments; their water content is too low for the intended application.

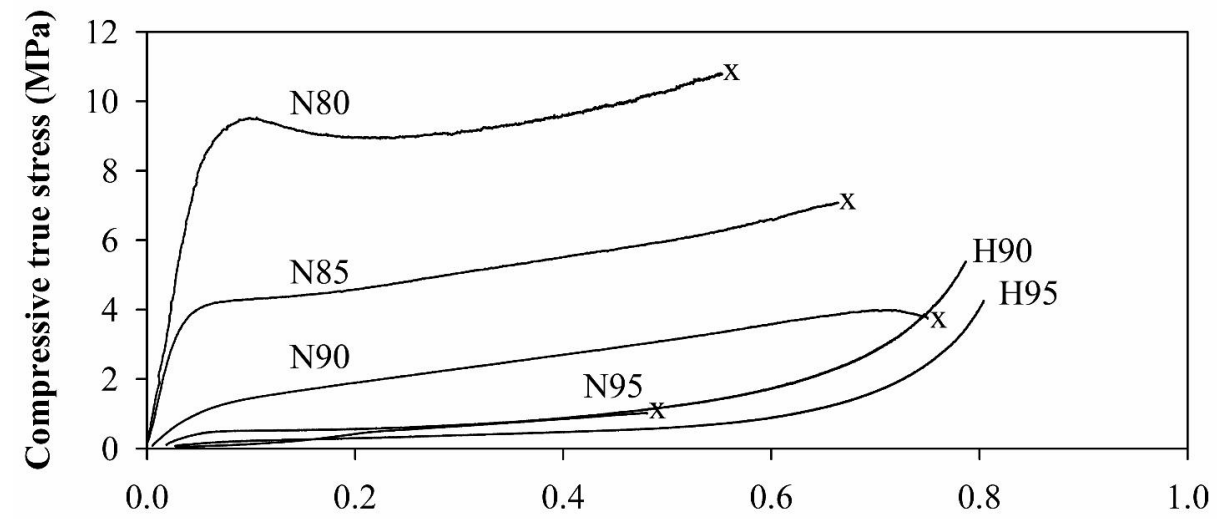

Compressive strain (-)

Figure 9: Stress-strain relationships obtained at a true strain rate of $3 \times 10^{-3}$ $s^{-1} . \mathrm{x}$ indicates that cracks were observed and compression was stopped.

The Young's modulus is calculated within the region of linear elastic deformation. The calculated Young's moduli for all samples are shown in Table 2. For both the NVP and HEMA-based hydrogels, a decrease in Young's modulus is observed with increasing concentration of the hydrophilic component.

Table 2: Hydrogel Young's moduli.

\begin{tabular}{ll}
\hline Code & $E(\mathrm{MPa})$ \\
\hline N95 & 1 \\
N90 & 24 \\
N85 & 123 \\
N80 & 250 \\
H95 & 3 \\
H90 & 13 \\
\hline
\end{tabular}

Finite element analyses have been performed by various groups and the Young's modulus used to model the nucleus is in the range of $0.2-4 \mathrm{MPa}[16$, 34-37]. This modulus should result in a physiological stress distribution. The 
compositions of both the NVP- and the HEMA-based hydrogels can be tuned to the desired modulus range. Both N95 and H95 are already within that range.

\subsection{Rheology}

The rheological properties of the natural human nucleus pulposus were determined by Iatridis et al. [38]. They found the magnitude of the complex shear modulus to be $11 \mathrm{kPa}$ and the phase angle to be 24 degrees at an angular frequency of $10 \mathrm{rad} / \mathrm{s}$. To compare our hydrogels with the natural nucleus, rheology experiments were performed.

The resulting complex shear modulus $\mathrm{G}^{*}$ and phase angle $\delta$ are depicted respectively in Figure $10 \mathrm{~A}$ and Figure $10 \mathrm{~B}$. In these figures the data of Iatridis [38] are also shown (NP).

In general, $\mathrm{G}^{*}$ decreases going from the glassy state to the rubbery state and reaches a constant value in the rubbery plateau. For the NVP-based hydrogels, the complex shear modulus is more or less independent of the frequency, implying that the hydrogels are in the rubbery plateau at the applied frequencies. On the other hand, for the HEMA-base hydrogels, the variation in $\mathrm{G}^{*}$ is about one order of magnitude, indicating that the rubbery plateau is not yet reached. This suggests that the glass transition temperature of the HEMAbased hydrogels is close to room temperature. Since it is impossible to determine the $T_{g}$ of hydrated hydrogels using DSC, due to the small range between the freezing and evaporation of water, it is tried to assess the $T_{g}$, based on the theoretical $\mathrm{T}_{g}$ of water $\left(-138{ }^{\circ} \mathrm{C}\right.$ [39]) and the $\mathrm{T}_{g}$ 's of the dry copolymers $\mathrm{H} 95\left(100^{\circ} \mathrm{C}\right)$ and $\mathrm{H} 90\left(93^{\circ} \mathrm{C}\right)$. These were determined analogous to the $\mathrm{T}_{\mathrm{g}}$ 's of the NVP-based copolymers. Using the EWC and the Fox equation, the theoretical glass transition is $-7.6^{\circ} \mathrm{C}$ for $\mathrm{H} 95$ and $18.2^{\circ} \mathrm{C}$ for $\mathrm{H} 90$. These values are indeed close to room temperature and this explains the frequency dependence of $\mathrm{G}^{*}$.

Passing $T_{g}$, upon going from the glassy to the rubbery state, the phase angle shows a maximum and decreases towards the plateau-region. Like for the modulus, relatively large frequency dependence is observed for the phase angle of the HEMA-based hydrogels. The phase angle decreases from about 55 degrees to 12 and 40 degrees for H95 and H90 respectively. For the NVP-based hydrogels, the phase angle is much less dependent on the frequency and is on average between 8 and 16 degrees. Low values of the phase angle $\delta$ indicate very elastic behaviour. 


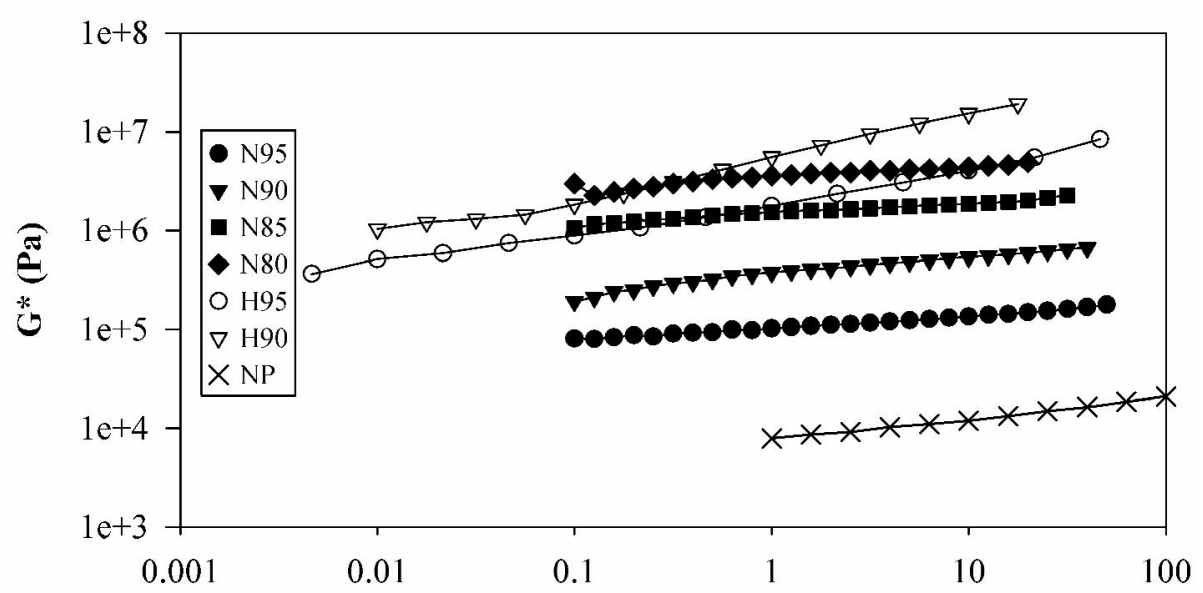

A

Frequency (rad/s)

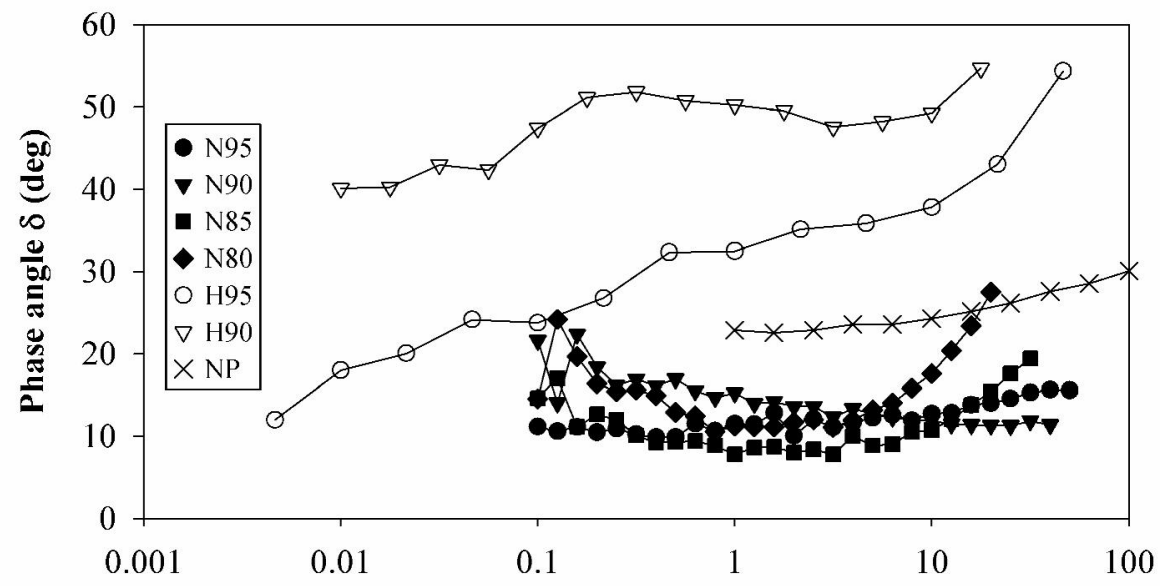

B

Frequency ( $\mathrm{rad} / \mathrm{s})$

Figure 10: Rheological spectra from a dynamic shear experiment at $0.01 \%$ strain; ( $A$ ) complex shear modulus $G^{*},(B)$ phase angle $\delta$.

From the lower complex shear modulus of the natural nucleus $\left(10^{4} \mathrm{~Pa}\right)$ compared to that of the hydrogels $\left(10^{5}-10^{7} \mathrm{~Pa}\right)$, it can be concluded that the natural nucleus has a more fluid consistency as compared to the soft solid character of the tested hydrogels. This result is in agreement with the finite element analysis, where it is also shown that, to obtain a physiological stress distribution, the synthetic nucleus should have a more solid-like character than the natural nucleus. 


\subsection{Hysteresis}

The natural nucleus pulposus is exposed to continuous cyclic loading. A preliminary cyclic compression experiment was performed to evaluate the response of the hydrogels on cyclic loading. The experiment involved 10 loading cycles to minimize dehydration effects. In this experiment N80 was also left out, because of its high stiffness. Results of N95, N90, H95, H90 are displayed in Figure $11 \mathrm{~A}$ and $11 \mathrm{~B}$. Results of N85 are not shown, but are comparable to N90, only the maximum stress is higher (6 MPa). Of course the amount of compression of the nucleus prosthesis is determined by the load it is subjected to and the stiffness of the implant material. Here we compressed to $30 \%$, but since all materials have a different stiffness, different forces are needed to achieve this strain. After a number of cycles, the hysteresis loops become repeatable, the materials are then preconditioned. Both $\mathrm{N} 90$ and $\mathrm{H} 90$ are considerably deformed during the first cycle, whereas N95 and H95 only deform to a small extent. It was noticed that, when given more time to recover in PBS, $\mathrm{H} 90$ returned almost fully to its original height. Clearly, these experiments provide only a first impression of the hysteresis characteristics. More extensive investigation on long-term fatigue of our hydrogels, submerged in PBS, is planned.

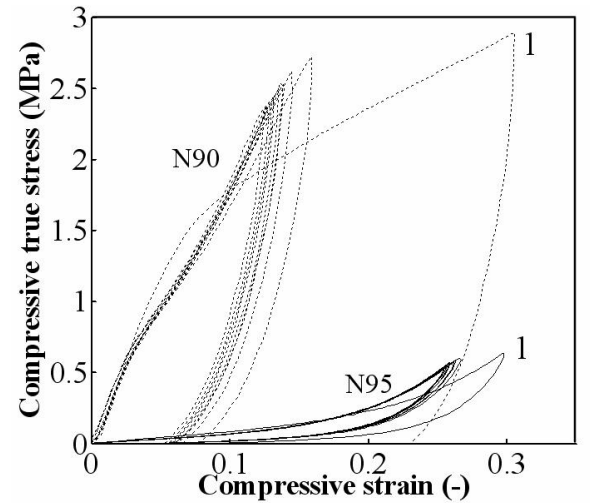

A

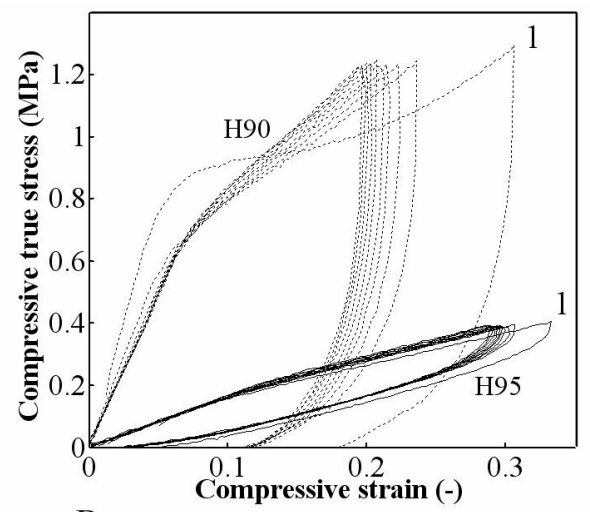

B

Figure 11: Hysteresis curves from a dynamic compression experiment, in which the hydrogels were compressed from 0 to 30\%; (A) NVP-based hydrogels, (B) HEMA-based hydrogels. 1 indicates the first cycle.

The area enclosed by a hysteresis loop is a measure for the dissipated energy. The energy dissipation of the HEMA-based hydrogels is larger than the energy dissipation of the NVP-based hydrogels, which is consistent with the higher phase angle found for these materials in the rheology experiments. 
Since N95 and H95 are within the desired stiffness range, it is expected that the stresses acting on them are representative for the natural situation. If these stresses (up to $0.4 \mathrm{MPa}$ ) would be applied on $\mathrm{N} 90$ and H90, the compression would be small and they would only deform elastically, since these forces are within their region of linear elasticity.

\section{Concluding remarks}

This study reveals that biocompatible, radiopaque hydrogels can be manufactured in such a way that they have adequate physical-mechanical properties to serve as a prosthesis for the nucleus pulposus. From the set of 8 different radiopaque hydrogels ( $\mathrm{N} 95, \mathrm{~N} 90, \mathrm{~N} 85, \mathrm{~N} 80, \mathrm{H} 95, \mathrm{H} 90, \mathrm{H} 85, \mathrm{H} 80$ ), the materials N95 and H95 appear to be most suitable. Swelling ability and rheological behaviour of N95 favors it above H95. N95 swells by $78 \%$ vs. $23 \%$ for H95, consequently N95 is smaller in dry form and thus easier to implant through a minimal incision in the annulus.

We foresee that radiopaque hydrogels as described here are attractive materials for replacement of a herniated nucleus pulposus, especially in those cases which allow for repair of the annulus ring: the annulus will be sutured or closed via a tissue engineering strategy. It is also conceivable that the radiopaque hydrogels are useful as an alternative to the Prosthetic Disc Nucleus of Raymedica, offering the advantage of intrinsic radiopacity (i.e. the implants are completely visible, and no X-ray markers are needed). Noteworthy, the new hydrogels can also be visualized readily and without artifacts by means of MRI [18]. Our future investigation will include fatigue testing and animal experiments. Also, implant geometry and implantation techniques will have to be studied to achieve a perfect fit of the implant in the nucleus cavity.

\section{Acknowledgements}

This research was funded by the Dutch Polymer Institute (DPI), project number 398, Intervertebral Disc Prosthesis. 


\section{References}

1. Andersson GB. Epidemiological features of chronic low-back pain. Lancet 1999; 354: 581-585.

2. Druss BG, Marcus SC, Olfson M, Pincus HA. The most expensive medical conditions in America. Health Aff (Millwood) 2002; 21: 105-111.

3. Bao QB, McCullen GM, Higham PA, Dumbleton JH, Yuan HA. The artificial disc: theory, design and materials. Biomaterials 1996; 17: 1157-1167.

4. Bao QB, Yuan HA. Prosthetic disc replacement: the future? Clin Orthop 2002: 139-145.

5. Szpalski M, Gunzburg R, Mayer M. Spine arthroplasty: a historical review. Eur Spine J 2002; 11 Suppl 2: S65-84.

6. Bao QB, Yuan HA. New technologies in spine: nucleus replacement. Spine 2002; 27: $1245-1247$.

7. Ghosh P. The biology of the intervertebral disc. vol. 1. CRC Press; 1988.

8. Thomas J, Lowman A, Marcolongo M. Novel associated hydrogels for nucleus pulposus replacement. J Biomed Mater Res 2003; 67A: 1329-1337.

9. Allen MJ, Schoonmaker JE, Bauer TW, Williams PF, Higham PA, Yuan HA. Preclinical evaluation of a poly (vinyl alcohol) hydrogel implant as a replacement for the nucleus pulposus. Spine 2004; 29: 515-523.

10. Bertagnoli R, Schonmayr R. Surgical and clinical results with the PDN prosthetic disc-nucleus device. Eur Spine J 2002; 11 Suppl 2: S143-148.

11. Klara PM, Ray CD. Artificial nucleus replacement: clinical experience. Spine 2002; 27: $1374-1377$.

12. Jin D, Qu D, Zhao L, Chen J, Jiang J. Prosthetic disc nucleus (PDN) replacement for lumbar disc herniation: preliminary report with six months' follow-up. J Spinal Disord Tech 2003; 16: 331-337.

13. Bertagnoli R, Vazquez RJ. The Anterolateral TransPsoatic Approach (ALPA): a new technique for implanting prosthetic disc-nucleus devices. J Spinal Disord Tech 2003; 16: 398-404.

14. Shim CS, Lee SH, Park CW, Choi WC, Choi G, Choi WG, Lim SR, Lee HY. Partial disc replacement with the PDN prosthetic disc nucleus device: early clinical results. J Spinal Disord Tech 2003; 16: 324-330.

15. Korge A, Nydegger T, Polard JL, Mayer HM, Husson JL. A spiral implant as nucleus prosthesis in the lumbar spine. Eur Spine J 2002; 11 Suppl 2: S149-153.

16. Meakin JR, Reid JE, Hukins DW. Replacing the nucleus pulposus of the intervertebral disc. Clin Biomech (Bristol, Avon) 2001; 16: 560-565.

17. Benzina A, Kruft MAB, Bar F, van der Veen FH, Bastiaansen CW, Heijnen V, Reutelingsperger $\mathrm{C}$, Koole $\mathrm{LH}$. Studies on a new radiopaque polymeric biomaterial. Biomaterials 1994; 15: 1122-1128. 
18. van Hooy-Corstjens CSJ, Aldenhoff YB, Knetsch ML, Govaert LE, Arin E, Erli H, Koole LH. Radiopaque polymeric spinal cages: a prototype study. Journal of Materials Chemistry 2004; 14: 3008-3013.

19. van Hooy-Corstjens CSJ, Govaert LE, Spoelstra AB, Bulstra SK, Wetzels GM, Koole LH. Mechanical behaviour of a new acrylic radiopaque iodine-containing bone cement. Biomaterials 2004; 25: 2657-2667.

20. Aldenhoff $Y B$, Kruft MAB, Pijpers AP, van der Veen FH, Bulstra SK, Kuijer R, Koole $\mathrm{LH}$. Stability of radiopaque iodine-containing biomaterials. Biomaterials 2002; 23: 881-886.

21. Kruft MAB, Koole LH. A convenient method to measure monomer reactivity ratios. Application to synthesis of polymeric biomaterials featuring intrinsic radiopacity. Macromolecules 1996; 29: 5513-5519.

22. Vistica DT, Skehan P, Scudiero D, Monks A, Pittman A, Boyd MR. Tetrazoliumbased assays for cellular viability: a critical examination of selected parameters affecting formazan production. Cancer Res 1991; 51: 2515-2520.

23. Mosmann T. Rapid colorimetric assay for cellular growth and survival: application to proliferation and cytotoxicity assays. J Immunol Methods 1983; 65: 55-63.

24. Shin H, Temenoff JS, Mikos AG. In vitro cytotoxicity of unsaturated oligo[poly(ethylene glycol) fumarate] macromers and their cross-linked hydrogels. Biomacromolecules 2003; 4: 552-560.

25. Eijkelkamp MF, van Donkelaar CC, Veldhuizen AG, van Horn JR, Huyghe JM, Verkerke GJ. Requirements for an artificial intervertebral disc. Int J Artif Organs 2001; 24: 311-321.

26. Greenley RZ. Q and e values for free radical copolymerizations of vinyl monomers and telogens. In: Brandrup J, Immergut EH (eds.). Polymer Handbook, $3^{\text {rd }}$ ed. New York: Wiley-Interscience; 1989.

27. Davis TP, Huglin MB. Studies on Copolymeric Hydrogels of N-Vinyl-2-Pyrrolidone with 2-Hydroxyethyl Methacrylate. Macromolecules 1989; 22: 2824-2829.

28. Elias H-G. An Introduction to Polymer Science. Weinheim: VCH; 1997.

29. Mark JE. Polymer Data Handbook. Oxford: Oxford University Press; 1999.

30. Boelen EJ. Novel iodine containing biomaterials for use in intraocular lenses. Eindhoven: Technical University Eindhoven; 2002. Master thesis.

31. Diakoumakos CD, Raptis I, Tserepi A, Argitis P. Free-radical synthesis of narrow polydispersed 2-hydroxyethyl methacrylate-based tetrapolymers for dilute aqueous base developable negative photoresists. Polymer 2002; 43: 1103-1113.

32. Beers KL, Boo S, Gaynor SG, Matyjaszewski K. Atom transfer radical polymerization of 2-hydroxyethyl methacrylate. Macromolecules 1999; 32: 57725776.

33. Refojo MF. Hydrophobic Interaction in Poly(2-hydroxyethyl Methacrylate) Homogeneous Hydrogel. Journal of Polymer Science; Part A-1 1967; 5: 31033113. 
34. Fagan MJ, Julian S, Siddall DJ, Mohsen AM. Patient-specific spine models. Part 1: Finite element analysis of the lumbar intervertebral disc--a material sensitivity study. Proc Inst Mech Eng [H] 2002; 216: 299-314.

35. Goel VK, Monroe BT, Gilbertson LG, Brinckmann P. Interlaminar shear stresses and laminae separation in a disc. Finite element analysis of the L3-L4 motion segment subjected to axial compressive loads. Spine 1995; 20: 689-698.

36. Lavaste F, Skalli W, Robin S, Roy-Camille R, Mazel C. Three-dimensional geometrical and mechanical modelling of the lumbar spine. J Biomech 1992; 25: 1153-1164.

37. Wang JL, Parnianpour M, Shirazi-Adl A, Engin AE. Viscoelastic finite-element analysis of a lumbar motion segment in combined compression and sagittal flexion. Effect of loading rate. Spine 2000; 25: 310-318.

38. Iatridis JC, Weidenbaum M, Setton LA, Mow VC. Is the nucleus pulposus a solid or a fluid? Mechanical behaviors of the nucleus pulposus of the human intervertebral disc. Spine 1996; 21: 1174-1184.

39. Hamaura T, Newton JM. Interaction between water and poly(vinylpyrrolidone) containing polyethylene glycol. Journal of Pharmaceutical Sciences 1999; 88: 1228-1233. 


\section{Preliminary Evaluation of New Intrinsically Radiopaque Hydrogels for Replacing the Nucleus Pulposus}

Erik J.H. Boelen, Catharina S.J. van Hooy-Corstjens, Marion J.J. Gijbels, Sjoerd K. Bulstra, André van Ooij, Lodewijk W. van Rhijn and Leo H. Koole

Adapted from:

Journal of Materials Chemistry 16 (2006) 824-828

A comment on this paper was written by James Mitchell Crow:

James M. Crow. Beating Back Pain. Chemistry World (2006) Vol. 3 nr. 2 (Appendix 4). 


\section{Abstract}

Treatment of early degenerative disc disease can, in some cases, be accomplished through implantation of a synthetic prosthesis for the nucleus pulposus. This treatment is attractive, since the annulus fibrosus -as well as the function of the disc- is preserved. This study reports on two new synthetic hydrogels which were specifically designed as fully radiopaque prosthetic nucleus biomaterials.

Moreover, the new materials were engineered in such a way that they swell in situ (i.e. after implantation) to such an extent that they will fill the entire nucleus cavity. We describe: (i) assessment of the biocompatibility of the new biomaterials in an in vivo animal model, (ii) implantation of the new prosthesis in an ex vivo animal model (porcine spine), followed by (iii), assessment of the visibility of the entire nucleus prosthesis through both CT and MRI. The results further substantiate the idea that the concept of implantation of a prosthesis for the nucleus pulposus can benefit from contemporary insights and developments of novel synthetic biomaterials with intrinsic radiopacity. 


\section{Introduction}

Degenerative disc disease (DDD) is one of the most common indications in spinal surgery. Currently, there is no real 'golden standard' to treat DDD. Nonoperative treatment has many proponents, presumably since operative treatment of DDD is usually complicated and the outcome may be unsure. In general, operative treatment of DDD implies spinal fusion: (part of) the affected disc is removed and the adjacent vertebrae are connected with mechanical fasteners. The separation of the vertebrae is usually secured with a cage or with an autologous or allograft bone graft. Spinal fusion however, locally impairs the range of motion of the spine. Consequently, neighboring segments are called upon to compensate for the immobilized section, and this might result in further problems [1-5].

An alternative treatment has emerged for patients with mild (early) DDD or for some trauma cases. This treatment is based on an implant that is designed to replace the diseased or deformed nucleus pulposus, and to mimic the function of the natural healthy nucleus pulposus, while preserving the annulus fibrosus [6-9]. Nucleus replacement is a minimally invasive procedure. In order to be considered for a nucleus replacement, certain inclusion criteria must be fulfilled: (i), DDD is manifested by morphologic changes of the nucleus; (ii), the annulus fibrosus must be competent; (iii), residual disc height is at least $5 \mathrm{~mm}$ [10]. Perhaps the most successful nucleus prosthesis is the PDN-Solo [11, 12], developed by Raymedica Inc. (Minneapolis, USA). This implant has a hydrogel core, which is packed in a woven polyethylene jacket.

We became interested in developing an alternative to the commercial PDN, based on our previous work on radiopaque biomaterials and hydrogels[13, 14]. We hypothesized that the PDN concept can be improved further, especially concerning radiovisibility, taking advantage of contemporary insights into new polymeric biomaterials which combine several desirable properties (e.g. intrinsic radiopacity, controllable swelling, controllable physical-mechanical properties) [15]. We reasoned that it should be possible to design and fabricate a synthetic nucleus prosthesis that meets the following requirements:

The prosthesis consists of a hydrogel that, like the healthy nucleus, but unlike the PDN, fills up the entire cavity that is enclosed by the annulus ring.

The prosthesis is implanted in dry form (xerogel), allowing the opening in the annulus to be small. After insertion, the implant is allowed to swell (expand) in situ. 
The prosthesis has excellent visibility through both X-ray fluoroscopy and magnetic resonance imaging (MRI), such that no artifacts occur.

The swollen hydrogel has adequate physical-mechanical properties and fatigue resistance. For instance, the prosthesis must resist continuous loading, and peak loading which occurs upon bending or lifting things[16, 17].

Herein, we report on two different hydrogel biomaterials that potentially fulfill the above criteria. The envisioned procedure is depicted schematically in Figure 1. Filling the entire cavity, evidently, makes the new implants more biomimetic as compared to the PDN.

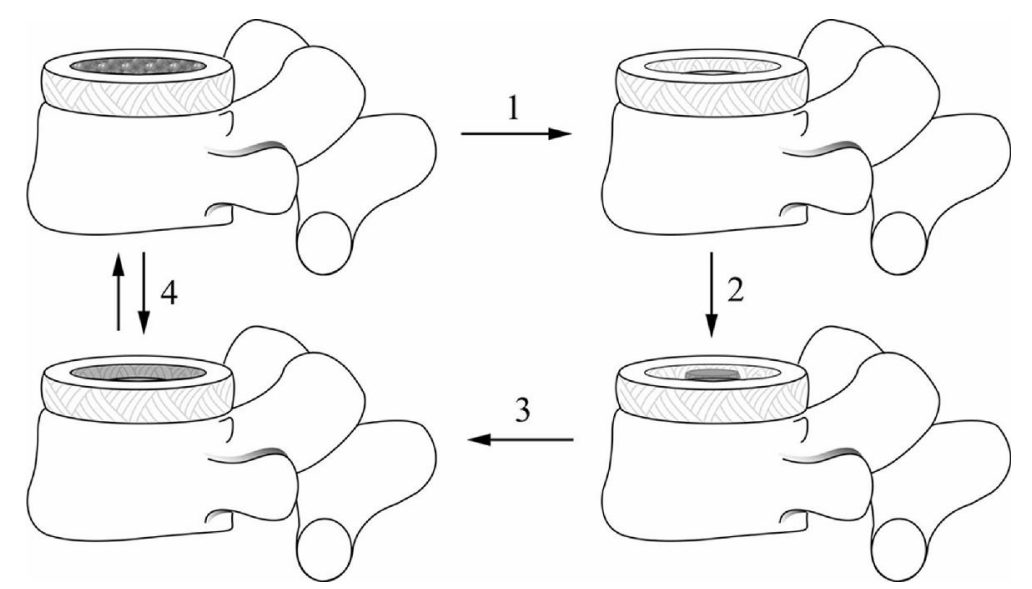

Figure 1: Schematic representation of nucleus replacement using a hydrogel: step 1 involves complete removal of the affected nucleus, step 2 is the insertion of the xerogel in the cavity, through a minimal incision in the annulus and step 3 is the subsequent in situ swelling to reach situation 4, in which the synthetic nucleus mimics the natural healthy situation.

Using both materials, nucleus prostheses prototypes were designed, manufactured and studied. We describe a series of experiments that reveal (i) biocompatibility in an in vivo model, (ii) implantability and in situ swelling, and (iii) visibility of the implant through X-ray and MR imaging. Moreover, the steps to take before the prosthesis can be introduced clinically are discussed briefly. 


\section{Materials and methods}

\subsection{Hydrogels}

Two hydrogels were prepared by copolymerizing either N-vinyl-2-pyrrolidinone (NVP) or 2-hydroxyethyl methacrylate (HEMA) with the radiopaque monomer 2(4'-iodobenzoyl)-oxo-ethyl methacrylate (4IEMA) $[18,19]$ in the molar ratio 94 : 6. We used 6 mol\% 4IEMA to slightly increase the radiopacity compared to the conclusions in previous work, wherein 5 mol\% 4IEMA was selected [15]. NVP and HEMA were purchased from Acros and distilled under reduced pressure before use. The monomer mixtures, together with $0.044 \mathrm{~mol} \%$ of 2,2'azobis(isobutyronitrile) (AIBN) were transferred to Teflon tubes. AIBN is the source of free radicals. The Teflon tubes were immersed in a temperature controlled oil bath. A temperature profile was run that keeps the oil bath for $8 \mathrm{~h}$ at $60^{\circ} \mathrm{C}$, followed by $4 \mathrm{~h}$ at $80^{\circ} \mathrm{C}$ and another $4 \mathrm{~h}$ at $100{ }^{\circ} \mathrm{C}$. The resulting copolymers are indicated by N94 (NVP / 4IEMA; 94/6) and H94 (HEMA / 4IEMA; 94/6). For both materials, the equilibrium water content (EWC) and volume swelling ratio (VSR) were determined in a swelling experiment in phosphate buffered saline (PBS) at room temperature. For this, the mass and dimensions of discs of the materials were monitored at various intervals, until equilibrium was reached. The VSR is the swollen volume divided by the dry volume and EWC is defined as the weight percentage of water in the swollen hydrogel at equilibrium. The Young's modulus of swollen cylindrical samples $(9 \mathrm{~mm}$ diameter, $9 \mathrm{~mm}$ height, after equilibrium swelling) of both hydrogels was determined from the initial slope of the stress-strain curve, measured in compression (strain rate $3 \times 10^{-3} \mathrm{~s}^{-1}$ ) using a Zwick 1445 compression bench with a 500N load cell in a water bath, which was kept at a constant temperature $\left(37^{\circ} \mathrm{C}\right)$.

\subsection{Biocompatibility in vivo}

The animal experiments were reviewed and approved by the Animal Ethical Committee of the University of Maastricht.

Dry copolymer discs of both $\mathrm{N} 94$ and $\mathrm{H} 94$ were sterilized with ethylene oxide and swollen in sterile PBS before implantation. Eight FVB mice of approximately 11 weeks old were anesthetized and operated under sterile conditions. Subcutaneous pockets were created on the back and in each pocket one 
swollen hydrogel disc of both $\mathrm{N} 94$ and $\mathrm{H} 94$ (4 mm diameter and $1 \mathrm{~mm}$ thickness) was implanted to study the tissue reaction to the materials. Ethanolsterilized PMMA discs of the same dimensions were used for comparison; PMMA is a proven biomaterial. Hence, each mouse received 3 implants.

Four mice were sacrificed after one week, the others after 3 months. At 5 weeks, X-ray images were taken of the remaining four mice. After sacrificing the mice, the materials and some of the surrounding tissue were removed and fixated in buffered formalin. Then the retrieved implants were dehydrated using a graded alcohol series and embedded in glycol methacrylate (Technovit 7100; Heraeus Kulzer, Germany). Subsequently the embedded specimens were sectioned $(3 \mu \mathrm{m})$ and stained with haematoxylin-eosin (HE). The histology was evaluated using light microscopy.

\subsection{Porcine intervertebral disc}

A lumbar spinal section was explanted from a recently deceased adult male pig. It was cleaned from soft tissues and conserved at $-80^{\circ} \mathrm{C}$. For implantation of the hydrogel, a disc was isolated by sawing adjacent vertebrae in their middle. After sawing, the spinal segment was allowed to thaw overnight. The size and shape of the porcine lumbar nucleus cavity was adapted from several cadaver specimens. The xerogels were machined to this shape, and their size was diminished to incorporate their swelling ability, so that their size after swelling matches the cavity size. Clinically, MRI can be employed to determine nucleus dimensions of the affected intervertebral disc in a patient, through discriminating annulus and nucleus by their water content ( $\mathrm{T}_{2}$-relaxation), enabling the selection of an implant with the exact shape and size.

\subsection{Hydrogel implantation}

The annulus of the isolated disc was cut using a surgical blade posterolateral. Two parallel incisions were made and one perpendicular incision, between the two incisions. This is to create a flap from the outer annulus layers. The flap was pulled away and a midannular incision was made through the annulus to reach the nucleus cavity (Figure 2). The gelly nuclear material was removed as much as possible with a small scoop. A prosthesis made from N94 was inserted using forceps. Care was taken to insert the xerogel properly into the nucleus cavity. Next, some PBS was injected into the cavity to initiate hydration of the implant. The annulus flap was sutured to close the defect. Then the entire 
segment was submerged in PBS and the implant was allowed to swell overnight.

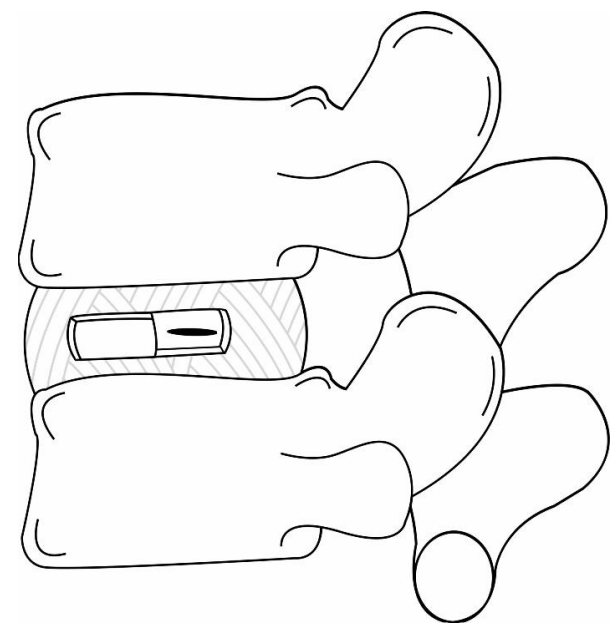

Figure 2: The annulus incision created to insert the nucleus prosthesis. After implantation the flap in the annulus is closed with suture.

\subsection{Visualization}

To investigate the position of the swollen hydrogel inside the nucleus cavity, CT and MRI scans were made. CT scans of the hydrogel had to prove if the X-ray contrast is sufficient after swelling, since the iodine concentration is lowered after swelling. Due to the high water content, the implant can also be clearly visualized using a $T_{2}$-weighted MRI sequence. After the scans, the disc was carefully sectioned through the annulus fibrosus to reveal the swollen implant and photographs were taken.

\section{Results and discussion}

\subsection{Hydrogels}

Thermal radical polymerization of the monomer mixtures resulted in clear glassy polymers. The resulting copolymers (N94 and H94) were washed prior to further analysis to get rid of any possible unreacted monomer.

The swelling ability of N94 is greater than H94, because NVP is more hydrophilic than HEMA. This can be expressed in terms of VSR or EWC (Table 
1). The EWC was determined at room temperature, but the difference in water content between room and body temperature is minimal $(<1 \%)$. The Young's modulus as determined in a compression experiment is also shown in Table 1. Mark that, though both hydrogels have quite different water contents, their moduli are almost equal. The Young's modulus is a measure for the material's stiffness, and for this application it is necessary to be in the range of $0.2-4$ $\mathrm{MPa}$ in order to mimic the mechanical behaviour of the natural nucleus pulposus[9, 20-22].

Table 1: Material properties.

\begin{tabular}{llll}
\hline Material & VSR $^{\mathbf{a}}(\mathbf{v} / \mathbf{v})$ & EWC $^{\mathbf{b}}(\mathbf{m} / \mathbf{m})$ & Young's modulus \\
\hline N94 & 3.9 & $74 \%$ & $0.7 \mathrm{Mpa}$ \\
H94 & 1.5 & $23 \%$ & $0.9 \mathrm{MPa}$ \\
\hline
\end{tabular}

${ }^{a}$ VSR: volume swelling rate. ${ }^{b}$ EWC: equilibrium water content.

\subsection{In vivo biocompatibility}

\section{Macroscopic observations}

The wound-healing process of the mice appeared to be normal and the implants could be observed as small bumps on the back. On the X-ray images both $\mathrm{N} 94$ and $\mathrm{H} 94$ can be distinguished (Figure 3), N94 at a slightly tilted angle. After retrieval of the implants, no changes in visual appearance or dimensions were observed after 3 months. Obviously, for these materials to serve as permanent nucleus prostheses, degradation is unacceptable.

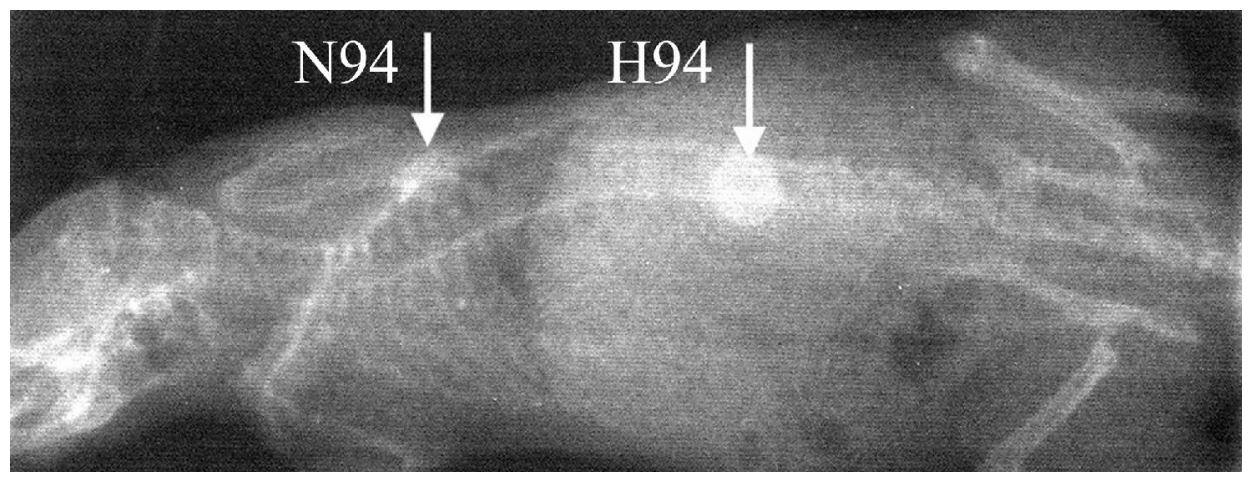

Figure 3: X-ray of the subcutaneously implanted hydrogel discs in a mouse. 


\section{Histology}

The one week follow-up of the subcutaneous implantation in mice showed mild acute inflammatory reactions and the start of a fibrous capsule for all materials. After 3 months, a thin fibrous capsule was present around all implants, containing linearly organized fibroblasts, aligned parallel to the implants. Figure 4 shows representative light micrographs of the materials after 3 months of subcutaneous implantation. The capsule thickness was comparable for all implants $( \pm 40 \mu \mathrm{m})$. A minimal inflammatory reaction was observed containing some macrophages and granulocytes and some newly formed blood vessels. Because PMMA is very stiff as compared to the surrounding embedded tissue, the fibrous capsule gets somewhat compressed during the cutting procedure. Basically, all 3 materials triggered only minimal and comparable tissue reactions. So both our swollen hydrogel materials N94 and H94 perform equally biocompatible as the proven hydrophobic biomaterial PMMA. The histological data of $\mathrm{N} 94$ and $\mathrm{H} 94$ are comparable to the results that were obtained previously by Aldenhoff et al., who studied long-term biocompatibility of analogous, but non-swelling radiopaque biomaterials [23].
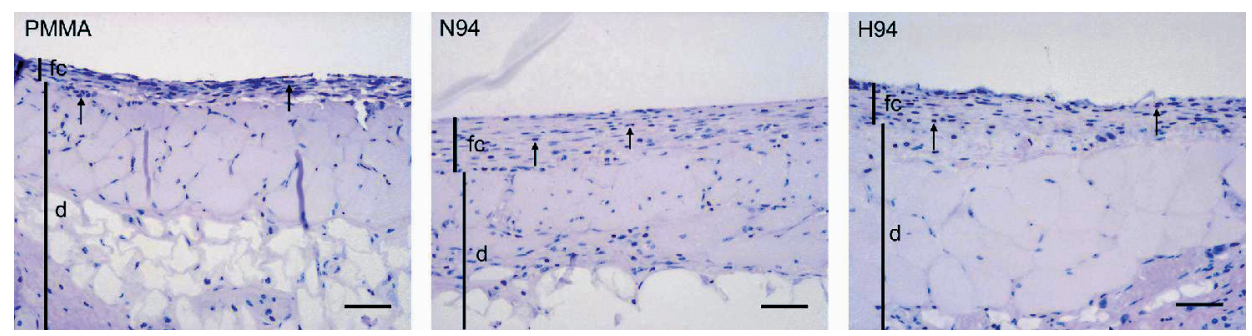

Figure 4: HE-staining of sections of the retrieved implants after 3 months. The materials are in the top of the image and a piece of the dermis (d) is seen underneath. Around all implants a thin fibrous capsule ( $f c$ can be observed and some inflammatory cells (arrows) are present. The scale bars represent 50 $\mu$ m.

\subsection{Porcine intervertebral disc}

The porcine nucleus is kidney-shaped with the dimensions: $25 \times 13 \times 4.5 \mathrm{~mm}$ $(\mid \times w \times t)$. To incorporate the swelling ability of N94 the dimensions of the implant were $15.5 \times 8 \times 2.8 \mathrm{~mm}$ (Figure 5). The time needed for this implant to reach equilibrium swelling is about one day. Once swollen, this implant will completely fill the cavity, left after removal of the gelly nucleus material. This is expected to result in optimal transmission of compressive forces onto the annulus fibers, thus mimicking the physiological state [20]. 


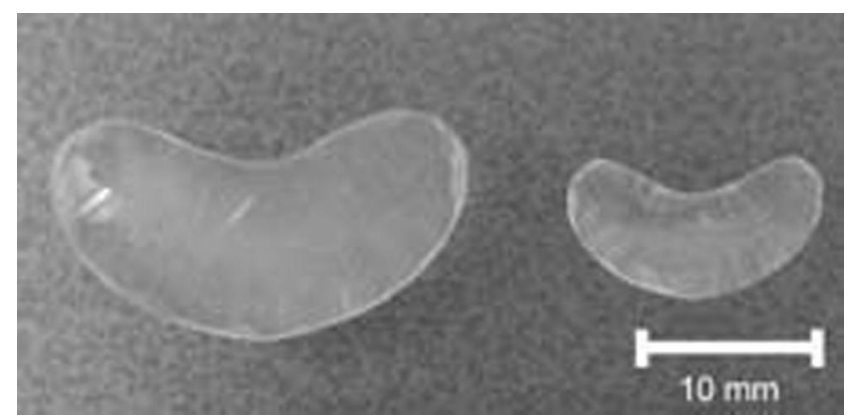

Figure 5: The swollen and dry hydrogel nucleus prosthesis. The shape is conforming to the porcine nucleus cavity.

\subsection{Hydrogel implantation}

Flap generation in the annulus fibrosus and aspiration of the natural nucleus pulposus proceeded smoothly. The nucleus prosthesis was inserted readily using a pair of tweezers, through the incision in the annulus fibrosus. Evidently, it must be inserted to align the curvature of the cavity. Through swelling pressure, the implant will probably position itself correctly within the empty nucleus cavity, since this is an unloaded situation. After implantation, PBS was injected into the cavity and the flap was sutured to close the annular defect. The implant will swell in situ to almost 4 times its original volume. This makes the implant much larger than the incision that was created in the annulus to implant the dry prosthesis and therefore extrusion is expected to be impeded.

\subsection{Visualization}

After one day, the swelling of an implant of the material N94 with the dimensions of the nucleus cavity is complete. CT and MRI scans were made of the porcine vertebrae containing the nucleus prosthesis. The CT and MRI image are shown in Figures $6 \mathrm{~A}$ and $6 \mathrm{~B}$ respectively. Both techniques can clearly distinguish the implant in the nucleus cavity. Apparently, the X-ray contrast of the swollen hydrogel is sufficient to visualize it in between two vertebrae. During surgery, X-ray fluoroscopy will probably be used instead of CT, but in terms of X-ray contrast there is no difference. Earlier, it was seen that these types of material can be visualized in situ using X-ray fluoroscopy [15]. CT provides extra information, since it is 3-dimensional. Also, due to the high water content, it can be clearly seen on the MRI image. An implant made out of H94 has a much lower water content, but will probably also appear on a MRI image: 
the difference in water content and subsequent $\mathrm{T}_{2}$-relaxation, between annulus and prosthesis, provides the contrast. On the contrary, due to its lower water content, H94 will exhibit more contrast using X-rays.

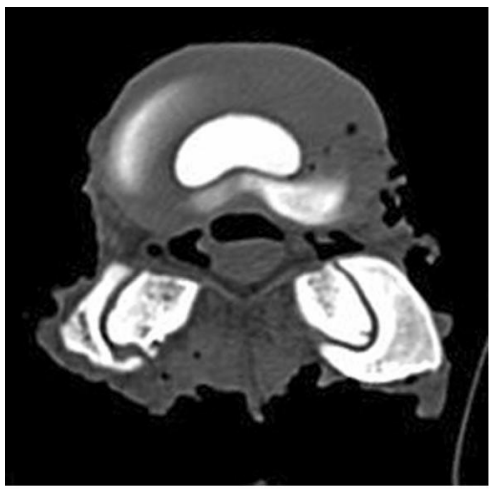

A

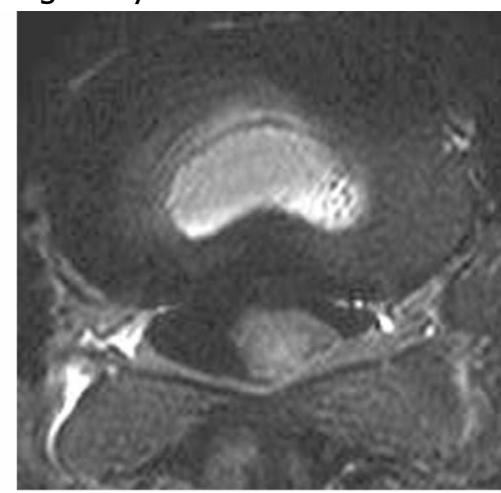

$\mathrm{B}$

Figure 6: Visualization of the hydrogel implant, using (A) CT and (B) $T_{2^{-}}$ weighted MRI. Both techniques clearly show the correctly positioned hydrogel implant.

Then, the intervertebral disc was sectioned to reveal the swollen hydrogel within the nucleus cavity (Figure 7). It confirmed the imaging techniques; the swollen implant completely filled the nucleus cavity.

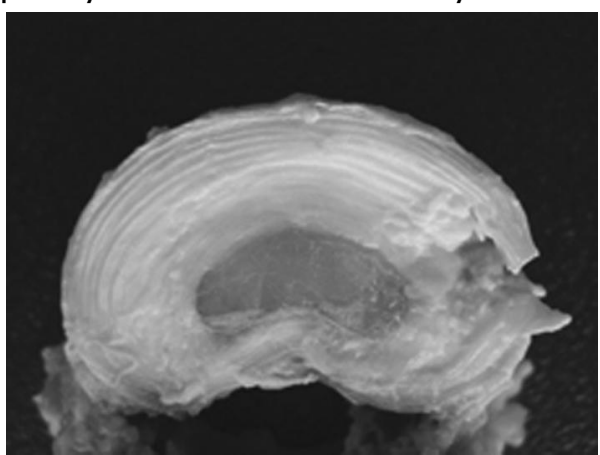

Figure 7: Picture of the swollen implant in the nucleus cavity; it perfectly fills the entire cavity.

\section{Concluding Remarks}

It is a challenge to design a radiopaque hydrogel that can withstand the harsh environment of the spinal column. Both radiopaque hydrogels, N94 and H94, 
fulfill a set of requirements to serve as prosthetic biomaterials for replacement of the nucleus pulposus. In particular, they offer the clinically significant advantage of integral visibility through CT and MRI. The absence of any artifacts in the MRI images is believed to be important, especially in view of the fact that there is a trend towards higher magnetic field strengths; these lead to improved quality of the MR images, but also to amplification of artifacts, such as those caused by metallic X-ray markers.

Moreover, it was verified that implants of $\mathrm{N} 94$ and $\mathrm{H} 94$ can be introduced into the nucleus cavity through a so-called annulus flap; this is an established technique in spinal surgery. N94 has a larger swelling capacity as compared to $\mathrm{H} 94$, which is theoretically beneficial. Still, extensive biomechanical tests will be performed in the near future, especially since implant extrusion and fatigue behaviour are important issues in this field. Preliminary fatigue tests seem promising. With our novel method we may decrease the risk of implant extrusion due to the improved and customized implant fit. Further experiments are planned to explore which material performs best in vivo. Biocompatibility of N94 and H94 was tested in an animal model (subcutaneous implantation for 3 months). Both materials were well tolerated, there were no signs of severe adverse tissue reactions. Histology of the tissues surrounding N94 and H94 were comparable to the histology of the tissues around the control material PMMA.

The results of this study provide further support for our earlier hypothesis, that these radiopaque hydrogels hold promise as improved biomaterials to replace a diseased or damaged nucleus pulposus in cases of a competent annulus fibrosus[15]. It is clear that more research is mandatory before clinical application of the new radiopaque hydrogels is possible. The nucleus prosthesis is a surgically invasive implant for long term use ( $>30$ days), which falls into Class IIb of the EC Medical Device Directive[24]. First, physical-mechanical testing for durability is necessary (fatigue resistance preferably in a confined model[25] and creep behaviour). Secondly, tests of the implant in a representative animal model need to be executed[26-29]. Thirdly, provided that the outcome of all experiments is positive, a clinical pilot study needs to be done. Then, the combined experimental data need to be examined by one of the Notified Bodies, which can then award the CE mark. It is important to consider that the two new radiopaque hydrogels are non-classical biomaterials, especially since 4IEMA was used as one of the building blocks. Another implant, the so-called ScrewFinder[30], which is also based on 4IEMA, already received a CE mark in 2001 (2012476CE01). 


\section{Acknowledgements}

This research was funded by the Dutch Polymer Institute (DPI), project number 398, Intervertebral Disc Prosthesis.

\section{References}

1. Ghiselli G, Wang JC, Bhatia NN, Hsu WK, Dawson EG. Adjacent segment degeneration in the lumbar spine. J Bone Joint Surg Am 2004; 86-A: 1497-1503.

2. Phillips FM, Reuben J, Wetzel FT. Intervertebral disc degeneration adjacent to a lumbar fusion. An experimental rabbit model. J Bone Joint Surg $\mathrm{Br} 2002$; 84: 289294.

3. Hilibrand AS, Robbins M. Adjacent segment degeneration and adjacent segment disease: the consequences of spinal fusion? Spine J 2004; 4: 190S-194S.

4. Bao QB, McCullen GM, Higham PA, Dumbleton JH, Yuan HA. The artificial disc: theory, design and materials. Biomaterials 1996; 17: 1157-1167.

5. Gillet P. The fate of the adjacent motion segments after lumbar fusion. J Spinal Disord Tech 2003; 16: 338-345.

6. Bao QB, Yuan HA. New technologies in spine: nucleus replacement. Spine 2002; 27: $1245-1247$.

7. Ray CD. The PDN prosthetic disc-nucleus device. Eur Spine J 2002; 11 Suppl 2: S137-142.

8. Husson JL, Korge A, Polard JL, Nydegger T, Kneubuhler S, Mayer HM. A memory coiling spiral as nucleus pulposus prosthesis: concept, specifications, bench testing, and first clinical results. J Spinal Disord Tech 2003; 16: 405-411.

9. Thomas J, Lowman A, Marcolongo M. Novel associated hydrogels for nucleus pulposus replacement. J Biomed Mater Res 2003; 67A: 1329-1337.

10. Guyer RD, Ohnmeiss DD. Intervertebral disc prostheses. Spine 2003; 28: S15-23.

11. Jin D, Qu D, Zhao L, Chen J, Jiang J. Prosthetic disc nucleus (PDN) replacement for lumbar disc herniation: preliminary report with six months' follow-up. J Spinal Disord Tech 2003; 16: 331-337.

12. Carl A, Ledet $E$, Yuan $H$, Sharan A. New developments in nucleus pulposus replacement technology. Spine J 2004; 4: 325S-329S.

13. van Hooy-Corstjens CSJ, Govaert LE, Spoelstra AB, Bulstra SK, Wetzels GM, Koole LH. Mechanical behaviour of a new acrylic radiopaque iodine-containing bone cement. Biomaterials 2004; 25: 2657-2667.

14. Peerlings CC, Hanssen HH, Bevers RT, Boelen EJH, Stelt BJ, Korthagen EJ, Koole LH. Heparin release from slippery-when-wet guide wires for intravascular use. J Biomed Mater Res 2002; 63: 692-698. 
15. Boelen EJ, van Hooy-Corstjens CS, Bulstra SK, van Ooij A, van Rhijn LW, Koole $\mathrm{LH}$. Intrinsically radiopaque hydrogels for nucleus pulposus replacement. Biomaterials 2005; 26: 6674-6683.

16. Ghosh P (ed.). The biology of the intervertebral disc. vol. 1. CRC Press; 1988.

17. White AA, Panjabi MM. Clinical biomechanics of the spine. Philadelphia: Lippincott; 1978.

18. Kruft MA, Benzina A, Blezer R, Koole LH. Studies on radio-opaque polymeric biomaterials with potential applications to endovascular prostheses. Biomaterials 1996; 17: 1803-1812.

19. van Hooy-Corstjens CSJ, Aldenhoff YB, Knetsch ML, Govaert LE, Arin E, Erli H, Koole LH. Radiopaque polymeric spinal cages: a prototype study. Journal of Materials Chemistry 2004; 14: 3008-3013.

20. Meakin JR, Reid JE, Hukins DW. Replacing the nucleus pulposus of the intervertebral disc. Clin Biomech (Bristol, Avon) 2001; 16: 560-565.

21. Fagan MJ, Julian S, Siddall DJ, Mohsen AM. Patient-specific spine models. Part 1: Finite element analysis of the lumbar intervertebral disc--a material sensitivity study. Proc Inst Mech Eng [H] 2002; 216: 299-314.

22. Meakin JR. Replacing the nucleus pulposus of the intervertebral disk: prediction of suitable properties of a replacement material using finite element analysis. J Mater Sci Mater Med 2001; 12: 207-213.

23. Aldenhoff YB, Kruft MAB, Pijpers AP, van der Veen FH, Bulstra SK, Kuijer R, Koole LH. Stability of radiopaque iodine-containing biomaterials. Biomaterials 2002; 23: 881-886.

24. Medical Devices Directive.

http://europa.eu.int/comm/enterprise/medical_devices/meddev/

25. Joshi A, Fussell G, Thomas J, Hsuan A, Lowman A, Karduna A, Vresilovic E, Marcolongo $M$. Functional compressive mechanics of a PVA/PVP nucleus pulposus replacement. Biomaterials 2006; 27: 176-184.

26. Wilke HJ, Kettler A, Claes LE. Are sheep spines a valid biomechanical model for human spines? Spine 1997; 22: 2365-2374.

27. Smit TH. The use of a quadruped as an in vivo model for the study of the spine biomechanical considerations. Eur Spine J 2002; 11: 137-144.

28. Reid JE, Meakin JR, Robins SP, Skakle JM, Hukins DW. Sheep lumbar intervertebral discs as models for human discs. Clin Biomech (Bristol, Avon) 2002; 17: 312-314.

29. Schimandle JH, Boden SD. Spine update. Animal use in spinal research. Spine 1994; 19: 2474-2477.

30. Bulstra SK, Benzina A, Kuijer R, Koole LH. Novel screw caps for use in orthopaedic and trauma surgery. Med Device Technol 1999; 10: 38-39. 


\section{Towards a Functional Radiopaque Hydrogel for Nucleus Pulposus Replacement}

Erik J.H. Boelen, Catharina S.J. van Hooy-Corstjens, Lodewijk W. van Rhijn and Leo $\mathrm{H}$. Koole

Adapted from:

Journal of Biomedical Materials Research. Part B, Applied Biomaterials. In press 


\section{Abstract}

Patients with severe back pain, attributed to a herniation of the nucleus pulposus of the intervertebral disc, can benefit from a replacement of only the nucleus pulposus, provided the annulus fibrosus is still functional. This study investigated four intrinsically radiopaque hydrogel biomaterials, which were designed specifically to replace the herniated nucleus pulposus. The important characteristic of these hydrogels is that they can be visualized entirely with both MRI and X-rays. The materials are based on copolymers of $\mathrm{N}$-vinyl-2pyrrolidinone (NVP) and/or 2-hydroxyethyl methacrylate (HEMA) and a radiopacity introducing monomer, 2-(4'-iodobenzoyl)-oxo-ethyl methacrylate (4IEMA). Two of the formulations also contain the chemical crosslinker allyl methacrylate (AMA). Physical-mechanical properties like the water-uptake, biocompatibility, stiffness, and fatigue and creep behaviour were studied, whilst keeping an eye on the intended application. All four materials were designed with 5-6 mass\% of iodine to ensure sufficient X-ray visibility in between two vertebrae. It was found that the materials display appropriate stiffness and biocompatibility. The crosslinked materials hold most promise as a functional nucleus prosthesis, as they combine these properties also with high water content, fatigue resistance, and recovery after loading. 


\section{Introduction}

Debilitating low back pain is an immense problem [1], with a myriad of pathologies. Very often, the actual cause is unknown [2], despite extensive investigations even with modern imaging techniques [3]. In cases where a specific diagnosis can be made, the pain can often be attributed to an intervertebral disc problem. Treatment of such patients is usually conservative (i.e., through exercise or physiotherapy). In severe cases however, surgery may be inevitable. When the patient suffers from Hernia Nucleus Pulposus (HNP) and the annulus fibrosus is still competent, except for the herniated part, it is sufficient to merely replace the nucleus pulposus $[4,5]$. HNP usually occurs at the lumbar level of the spine. Also in cases of early degenerative disc disease (DDD), replacing the nucleus pulposus can be an option. Nucleus replacement is an emerging approach. It preserves the remaining disc structures, i.e. the annulus fibrosus and the endplates, provided they are still functional. Indications and contraindications are very important to consider [6] and the decision for a nucleus replacement is left to the discretion of the surgeon.

Several designs already exist to serve as a nucleus replacement, using a variety of approaches [7-13]. Previously, we have advocated that improved prostheses for the nucleus pulposus can be made on the basis of radiopaque biocompatible hydrogels with intrinsic radiopacity $[14,15]$. These should be designed and shaped in such a way that they completely fill (after swelling) the cavity that is confined by the annulus fibrosus and the upper and lower endplates. Two promising radiopaque hydrogels emerged from our previous work: (i), the copolymer of N-vinyl-2-pyrrolidinone (NVP) and 2-(4'-iodobenzoyl)-oxo-ethyl methacrylate (4IEMA) with molar ratio NVP : 4IEMA $=94: 6$, this material is abbreviated as N94; (ii), the copolymer of 2-hydroxyethyl methacrylate (HEMA) and 4IEMA with molar ratio HEMA : 4IEMA $=94: 6$, this material is abbreviated as H94 [15]. Both materials were implantable, and they featured excellent biocompatibility and radiopacity.

To date, no official test methodologies have been specified for nucleus pulposus replacements [16]. Here, we focus specifically on the stiffness, creep behaviour and recovery of $\mathrm{N} 94$ and H94. Furthermore, we slightly adapted both compositions to overcome two residual drawbacks of both materials: (i) NVP and NVP-oligomer leaching from N94 during swelling, directly after synthesis, and (ii) limited swelling and water uptake of H94. Both goals were achieved through the use of the crosslinker allyl methacrylate (AMA). 
We describe two new radiopaque hydrogel biomaterials which are believed to be optimal for the intended application as a nucleus pulposus prosthesis at the lumbar level: the crosslinked version of N94, and a crosslinked terpolymer of NVP, HEMA and 4IEMA. Physical-mechanical properties of these novel biomaterials, i.e., water diffusion, swelling, stiffness, fatigue resistance, and recovery, as well as the results from biocompatibility assays in vitro, are described.

\section{Materials and methods}

\subsection{Materials}

All chemicals were purchased from Acros (Landsmeer, The Netherlands). Both NVP and HEMA were distilled under reduced pressure to remove inhibiting additives. The monomer 4IEMA was synthesized as described before [17]. The crosslinker allyl methacrylate (AMA) was used as received, and 2,2'azobis(isobutyronitrile) (AIBN) was used as the source of free radicals in the temperature controlled bulk polymerization. Monomers and $0.03 \mathrm{~mol} \%$ of AIBN were mixed and transferred to Teflon tubes, which were closed with a stopper on one end. Then they were immersed in a thermostated oil bath and the temperature profile as depicted in Figure 1 was run. Four different materials were prepared as indicated in Table 1 . The polymerization yielded transparent, glassy rods, which were machined to discs or cylinders for the different analysis. All resulting polymers contain 5-6 mass\% iodine, which was found to be sufficient for visualizing the resulting hydrogels in between two vertebrae [14]. 


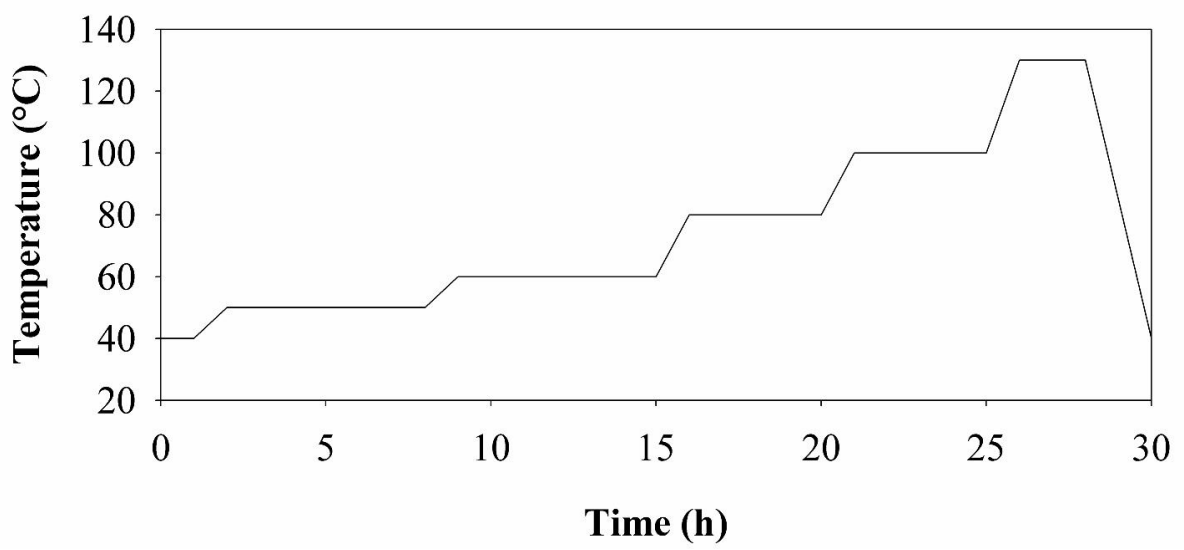

Figure 1: Temperature profile of the oil bath used for the radical polymerization.

Table 1: Material composition in molar percentages.

\begin{tabular}{lllll}
\hline Material & NVP & HEMA & 4IEMA & AMA \\
\hline N94 & 94 & - & 6 & - \\
H94 & - & 94 & 6 & - \\
N94_2 & 92.1 & - & 5.9 & 2 \\
T94_2 & 71.8 & 20.4 & 5.8 & 2 \\
\hline
\end{tabular}

\subsection{Swelling and diffusion}

Swelling of the different materials was studied by placing three discs of each material $(n=3)$ in ample phosphate-buffered saline (PBS) $\left(37^{\circ} \mathrm{C}\right)$ and weighing them frequently at different time intervals, after removing the surface water with a tissue. The aspect ratio of the discs (diameter/thickness) was more than 10 , so one-dimensional penetrant diffusion could be assumed [18]. The water content is defined as the mass percentage of water in the swollen disc. It was noted that the mass increase already levelled to a plateau value after $3 \mathrm{~h}$. It could not be excluded however, that some release of low-molecular mass extractables occurs at this stage [14]; we assumed that full equilibration is achieved after 2 weeks. Therefore, the equilibrium water content (EWC), as well as the volume swelling ratio (Q) of every material was determined after 2 
weeks of incubation. After 2 weeks the materials were weighed once more and their dimensions were measured. Subsequently, the discs were dried. The equilibrium water content (EWC) was calculated, using the material masses after 2 weeks of swelling and the dried mass. The volume swelling $\mathrm{Q}$ was determined by dividing the swollen volume by the dried volume. However, all samples were somewhat curved after drying, so sometimes it proved difficult to obtain true dimensions.

\subsection{Cell viability}

To evaluate the cytotoxicity of the materials, three dried, prewashed discs were UV-sterilized and swollen in medium for 2 days. Then, 3T3 mouse fibroblasts were placed on the surface of the hydrogel discs $\left(10^{4}\right.$ cells $/ \mathrm{cm}^{2}$, day 1$)$. At day 2 , the medium was refreshed and at day 3 the adhering cells were evaluated using the Live/Dead assay (Molecular Probes, Viability/Cytotoxicity Kit (L-3224)) by fluorescence microscopy (Nikon Eclipse E800 equipped with a Coolsnap camera (RS Photometric, USA)) [19].

\subsection{Static mechanics}

In order to determine basic mechanical properties of the materials, cylindrical samples (15 mm diameter, $15 \mathrm{~mm}$ height), swollen to equilibrium in water, were compressed to failure at a strain rate of $3 \times 10^{-3} \mathrm{~s}^{-1}$. Compression was performed in a waterbath at $37^{\circ} \mathrm{C}$, on a Zwick 1445 tension-compression tester using a $500 \mathrm{~N}$ load cell $(n \geq 3)$. The Young's modulus was determined from the linear slope of the resulting compression curves (up to $8 \%$ compressive strain).

\subsection{Dynamic mechanical analysis}

Swollen, cylindrical samples (15 mm diameter, $15 \mathrm{~mm}$ height) of the materials were subjected to a sinusoidal loading profile at several frequencies in the range 0.1 to $5 \mathrm{~Hz}(\mathrm{n} \geq 3)$. The mean compression level was $1.5 \mathrm{~mm}$ and the amplitude was $0.5 \mathrm{~mm}$. Hence, compressive strain was within the region of linear elasticity $(<15 \%)$. The tests were preformed on a MTS 810 Elastomer Test System, equipped with a $2.5 \mathrm{kN}$ load cell, in a $37{ }^{\circ} \mathrm{C}$ water bath and yielded the complex modulus $\mathrm{E}^{*}$ and phase angle $\delta$. 


\subsection{Rheology}

For rheological characterization of the hydrogels, thin discs $(2 \mathrm{~mm})$, swollen to equilibrium, were used with an aspect ratio larger than 10 . The experiments were preformed on a parallel plate viscometer (ARES 3LSLC1, Rheometric Scientific) at $37^{\circ} \mathrm{C}(\mathrm{n}=3)$. The material was compressed axially between to parallel plates to $0.18 \mathrm{~N}$ and PBS was applied around the edge to prevent dehydration. Also a small hood was placed over the setup. At first a strain sweep $(0.005 \%-0.5 \%)$ was performed at an angular frequency of $10 \mathrm{rad} / \mathrm{s}$. It was concluded that $0.01 \%$ strain was within the region of linear elasticity, so a dynamic frequency sweep was performed at this strain from $1-100 \mathrm{rad} / \mathrm{s}$ $(n=3)$, to yield material data like complex shear modulus $G^{*}$ and phase angle $\delta$.

\subsection{Creep and fatigue}

The creep experiments were performed on the same system as the static mechanical tests. The samples were compressed to $0.1 \mathrm{MPa}$ engineering stress for $3 \mathrm{~h}$, followed by a force of $0 \mathrm{~N}$ for $1 \mathrm{~h}$, to monitor their recovery. Sample heights before and after the tests were measured using a digital calliper. The compression was chosen to be $0.1 \mathrm{MPa}$, which is the physiologic swelling pressure of the intervertebral disc [20,21].

Fatigue resistance was checked using the same test system as for the DMA, but with a $25 \mathrm{kN}$ load cell this time. At a frequency of $5 \mathrm{~Hz}$ it was verified if cylindrical samples of the swollen material survive $10^{6}$ cycles of compression from $0.1 \mathrm{MPa}$ engineering stress to $15 \%$ strain $(n=3)$. One million cycles was chosen as a preliminary indication of fatigue resistance. To keep contact between the sample and the indenter, the test was preformed force controlled. Therefore, before starting the experiment, the indenter was lowered to $15 \%$ compression and the force needed was recorded. This force was then used as upper limit.

\subsection{Statistical analysis}

The results of the hydrogel properties were statistically analyzed using one-way ANOVA, with the Bonferroni correction, with a value of $p<0.05$ taken to be significant. 


\section{Results}

\subsection{Swelling and diffusion}

The water uptake of the materials in $37^{\circ} \mathrm{C}$ PBS was meticulously followed in time. The masses of the discs were measured regularly. The water content as a function of time is plotted in Figure 2. The materials swell isotropically. The difference in water content of the materials in water versus PBS at $37^{\circ} \mathrm{C}$, is smaller than $1 \%$.

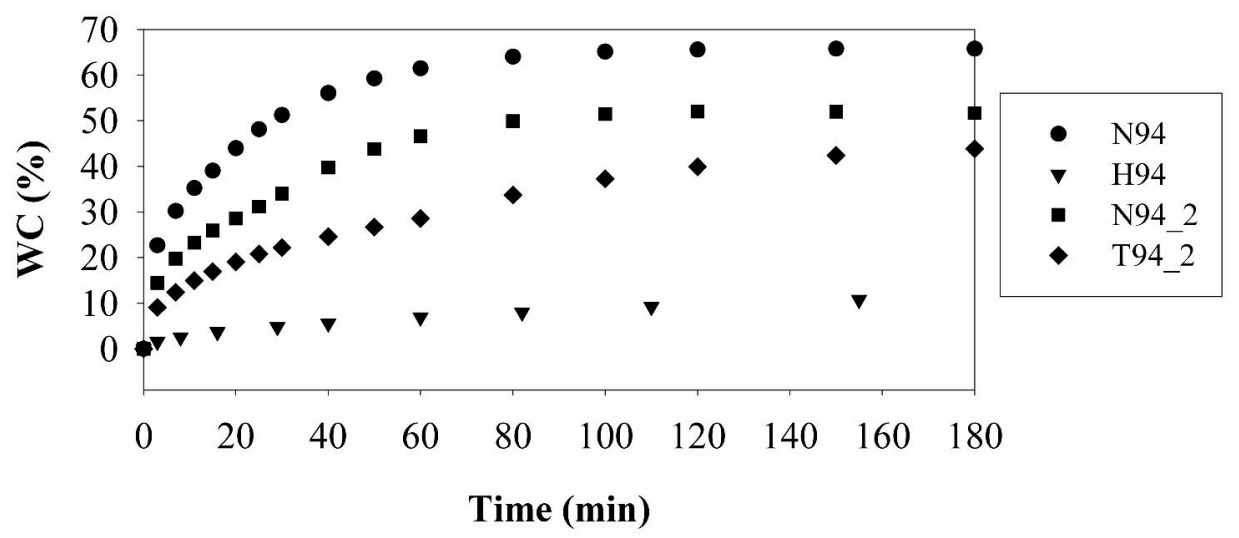

Figure 2: Swelling curves of the polymers in PBS at $37^{\circ} \mathrm{C}$ as a function of time.

Figure 2 shows that water uptake is relatively fast for the NVP-based materials N94 and N94_2: a plateau level is reached within $2 \mathrm{~h}$ of incubation. Swelling of the HEMA-based hydrogels is somewhat slower; a plateau is reached after $3 \mathrm{~h}$. As expected, the NVP-based hydrogels absorb more water in comparison with the HEMA-based materials. The data in Figure 2 were used to calculate several parameters that characterize the diffusion process for each of the materials: (i) the diffusional exponent ( $n$ ), and (ii) the diffusion coefficient (D). The values for $\mathrm{n}$ were calculated with the formula:

$$
F=\frac{M_{t}}{M_{\infty}}=k t^{n}
$$


where $M_{t}$ and $M_{\infty}$ are the mass of water in the sample at time $t$ and at equilibrium hydration respectively, $k$ is a constant and $n$ is the diffusional exponent. The swelling data were fitted to equation 1 and the diffusional exponent $n$ was deduced from the initial part $\left(M_{t} / M_{\infty}<0.6\right)$ of the resulting graph (Table 2). From the values for $n$ can be concluded that the uptake of water is mainly diffusion controlled $(n \approx 0.5)$. Now, the diffusion coefficient $D$ can be calculated from the short time approximation of Fick's law for thin sheets [22, 23],

$$
\frac{M_{t}}{M_{\infty}}=4\left(\frac{D t}{\pi l^{2}}\right)^{0.5}
$$

where $/$ is the initial thickness of the sheet. Table 2 shows the diffusion coefficient $D$ for the different materials. These data reflect, on a quantitative basis, that water uptake is fastest for the NVP-based hydrogels.

After 2 weeks in PBS $\left(37^{\circ} \mathrm{C}\right)$, the materials were weighed and measured once more and dried. From this, the EWC, volume swelling $\mathrm{Q}$ and mass loss of the different materials are determined (Table 2). The data are all significantly different.

Several conclusions can be drawn from this data: (i) material N94 loses a substantial amount of extractables during the 2 weeks of incubation, as is evident from its mass loss (40.2\%). This is a serious disadvantage, which disqualifies N94 for the intended use as a prosthesis for the nucleus pulposus. (ii) Material H94 does not show any mass loss during incubation. In part, this observation is not surprising, since it is know that free radical polymerization of HEMA leads to the formation of three dimensional crosslinked networks. The existence of such a network could also explain why the EWC of H94 is relatively low (22.4\%): swelling requires the expansion of the 3D network, which becomes progressively more difficult as the network density increases. Moreover, (co)polymers of HEMA are known to be less hydrophilic than (co)polymers derived from NVP. With respect to the intended application, it can be concluded that $\mathrm{H} 94$ is not suitable, since swelling is insufficient. (iii) Material N94_2 overcomes much of the principal drawback of N94, in the sense that the loss of mass via extractables is reduced substantially, i.e. from approximately $40 \%$ to approximately $12 \%$. This effect is solely due to crosslinking. Interestingly, the EWC of N94_2 is still high (55\%). With respect to the application, the loss of $12 \%$ of the mass during swelling is still a drawback, in view of the toxic effect that such extractables inflict. Application of N94_2 
would only be possible after a pre-wash step. (iv) The terpolymer T94_2 represents a further improvement of N94_2, in terms of further reduction of the mass loss, while the EWC is still relatively large. Note that T94_2 still loses some extractables during the 2 weeks of incubation, approximately $2 \%$. This might still imply that a pre-wash step is required to avoid any toxic reaction.

Table 2: Data determined from the swelling experiment in PBS at $37^{\circ} \mathrm{C}$.

\begin{tabular}{|c|c|c|c|c|c|c|}
\hline Material & $\begin{array}{l}\text { EWC } \\
\text { (wt.\%) }\end{array}$ & $\mathbf{Q}$ & $\begin{array}{l}\text { Mass } \\
\text { (wt.\%) }\end{array}$ & loss & $n$ & $\begin{array}{lll}D & \times & 10^{5} \\
\left(\mathrm{~cm}^{2}\right. & \left.\mathrm{min}^{-1}\right)\end{array}$ \\
\hline N94 & $73.5 \pm 0.1$ & $3.7^{*}$ & 40.2 & & 0.55 & 2.9 \\
\hline H94 & $22.4 \pm 0.6$ & 1.4 & 0 & & 0.51 & 0.7 \\
\hline N94_2 & $55.0 \pm 0.1$ & 2.1 & 11.8 & & 0.48 & 3.2 \\
\hline T94_2 & $46.1 \pm 0.1$ & 2.0 & 2.3 & & 0.47 & 2.0 \\
\hline
\end{tabular}

\subsection{Cell viability}

Figure 3 shows micrographs, obtained with the Live/Dead assay. The surfaces contain mostly live cells (green) and sporadically a dead cell (red). Although cells prefer more hydrophobic surfaces [24], some cell spreading on the surface can be observed. Only the new, crosslinked materials were evaluated, since the biocompatibility of $\mathrm{N} 94$ and $\mathrm{H} 94$ was already demonstrated in vivo in a mouse model, by subcutaneous implantation [15]. The addition of the crosslinker AMA to the material (N94_2), or the crosslinked combination of both N94 and H94 (T94_2), does not show any adverse effects on the cytocompatibility and is not expected to have ill effects on the biocompatibility. The intervertebral disc is the largest avascular structure in the body [25], and the cell density in the nucleus pulposus is low [26]. Nonetheless, for a nucleus replacement, cytocompatibility is very important, also in view of the fact that the delicate spinal cord is in the proximity of the implant. 

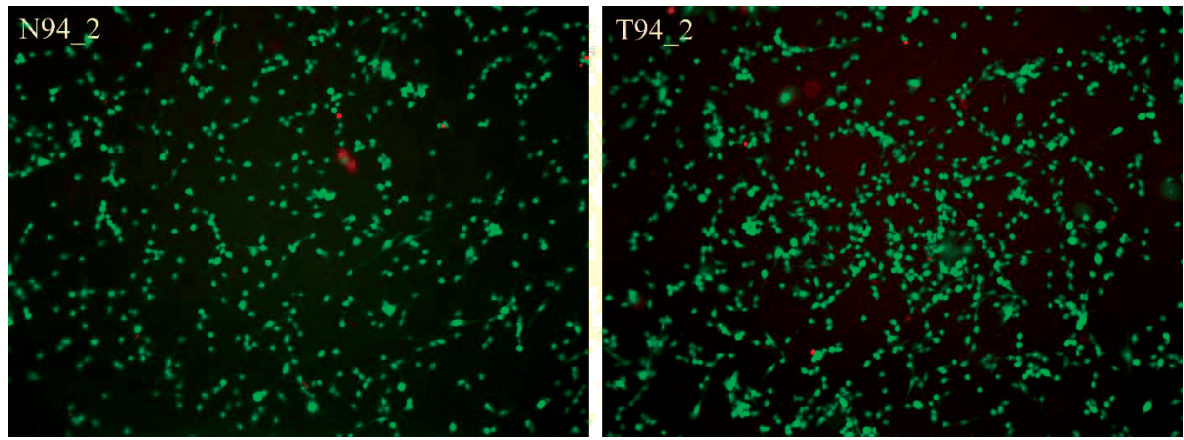

Figure 3: Micrographs obtained with fluorescence microscopy in the Live/Dead staining assay of $3 T 3$ mouse fibroblast cells directly on the surface of the hydrogels.

\subsection{Static mechanics}

Figure 4 shows some typical compression curves of the materials. True stress is plotted versus the strain. The materials behaviour for all materials, except N94, is linear to at least $30 \%$, which is twice the physiologic compression of the intervertebral disc [21]. N94 shows non-linear behaviour beyond 12\% compression. Since, the physiologic compression is $15 \%$, it is mandatory that the materials behave linearly at least up to this strain, so no permanent deformation occurs. Also, from the reported stiffness of the lumbar intervertebral disc $(2000 \mathrm{~N} / \mathrm{mm})$ and the maximal force $(8 \mathrm{kN})$, a maximal compression of $40 \%$ is expected [21, 27].

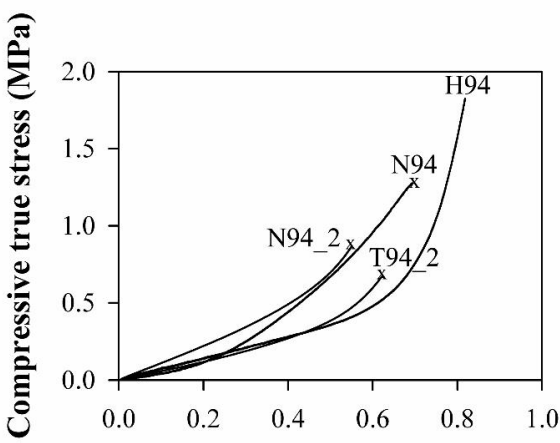

A
$\mathrm{B}$

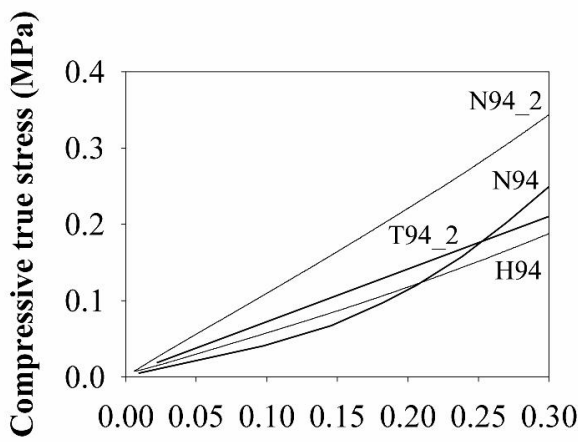

Compressive strain (-)

Figure 4: Stress-strain relationships obtained at a compressive strain rate of 3 $\times 10^{-3} s^{-1}$. For each material, a representative curve is shown $(A) . x$ indicates material failure. Note in the expansion (B), the linearity for H94, N94_2 and T94_2 up to 30\% compression. 
All materials can be compressed to over 50\%. H94 did not break, even at $80 \%$ compressive strain. Table 3 shows the moduli, calculated from part of the linear region (up to $8 \%$ ) in the compression curves and the strains at failure. It is clear that crosslinking adds to the stiffness of the hydrogels at equilibrium.

Table 3: Stiffness and strain at failure of the hydrogels, when compressed at a strain rate of $3 \times 10^{-3} \mathrm{~s}^{-1}\left(37^{\circ} \mathrm{C}\right)$.

\begin{tabular}{lll}
\hline Material & E (MPa) & $\begin{array}{l}\text { Compressive strain } \\
\text { at failure (-) }\end{array}$ \\
\hline N94 & $0.6 \pm 0.1$ & $0.69 \pm 0.04$ \\
H94 & $0.8 \pm 0.1$ & - \\
N94_2 & $1.5 \pm 0.1$ & $0.54 \pm 0.06$ \\
T94_2 & $1.1 \pm 0.1$ & $0.58 \pm 0.03$ \\
\hline
\end{tabular}

\subsection{Dynamic Mechanical Analysis}

Since the intervertebral disc is a very dynamic structure, it was also deemed necessary to subject the materials to dynamic mechanical analysis (DMA).

From the dynamic sinusoidal loading profile, the complex modulus $\mathrm{E}^{*}$ and the phase angle $\delta$ were derived for several frequencies in the range 0.1 to $5 \mathrm{~Hz}$. It was noted that the materials behave stiffer when the frequency is higher; a higher strain rate gave higher moduli (data not shown). Also the phase angle increased slightly with increasing frequency, except for N94. The physiologic frequency $1 \mathrm{~Hz}$ was of special interest. The values obtained at this frequency for $\mathrm{E}^{*}$ and $\delta$ are displayed in Table 4 . The complex moduli correspond qualitatively to the data on the Young's moduli (Table 3), although the values of $\mathrm{E}^{*}$ are all slightly higher, which is caused by the higher strain rate.

Also displayed in Table 4 are values for the nucleus pulposus (NP) of sheep, determined by Leahy et al. [28]. The sheep nucleus is very similar to the human nucleus [29]. Clearly, our materials are much stiffer (compare $E^{*}$ values). The phase angle ( $\delta=18 \mathrm{deg}$ ) corresponds with the $\delta$-value of the uncrosslinked materials N94 and H94, but is higher than the $\delta$-values of N94_2 and T94_2. These crosslinked materials display a higher degree of elasticity, i.e. they have a low phase angle, so they are expected to have better recovery when subjected to loading. 
Table 4: Material parameters obtained at $1 \mathrm{~Hz}$, using DMA and rheology.

\begin{tabular}{|c|c|c|c|c|}
\hline \multirow{2}{*}{ Material } & \multicolumn{2}{|l|}{ DMA } & \multicolumn{2}{|c|}{ Rheology } \\
\hline & $\mathrm{E}^{*}(\mathrm{MPa})$ & $\delta(\mathrm{deg})$ & $\mathrm{G}^{*}(\mathrm{kPa})$ & $\delta$ (deg) \\
\hline N94 & 1.02 & 20.7 & 31.7 & 16.9 \\
\hline H94 & 1.19 & 17.6 & 63.5 & 19.9 \\
\hline N94_2 & 2.01 & 6.3 & 48.8 & 7.7 \\
\hline T94_2 & 1.48 & 6.3 & 80.8 & 6.0 \\
\hline NP & $0.07^{\#}$ & $18^{\#}$ & $11^{\$}$ & $24^{\$}$ \\
\hline $\begin{array}{l}\text { \# Data fro } \\
\$ \text { Data fro }\end{array}$ & $\begin{array}{l}\text { ny et al. } \\
\text { dis et al. }\end{array}$ & & & \\
\hline
\end{tabular}

\subsection{Rheology}

Material parameters that were derived from the rheological experiments are the complex shear modulus $G^{*}$ and the phase angle $\delta$. To compare with the data obtained with DMA, the frequency was expressed in $\mathrm{Hz}$ instead of radians. The complex shear modulus and phase angle are depicted in Figures $5 \mathrm{~A}$ and $5 \mathrm{~B}$ respectively. The values of both $\mathrm{G}^{*}$ and $\delta$ at $1 \mathrm{~Hz}$ are added to Table 4. The phase angles, measured using both techniques, are very comparable.

Rheological properties of the natural human nucleus pulposus at $10 \mathrm{rad} / \mathrm{s}$ (1.6 $\mathrm{Hz}$ ) are also displayed in Table 4 (data from Iatridis et al. [30]).

All of our materials have $\mathrm{G}^{*}$ values that, by far, exceed $\mathrm{G}^{*}$ of the human nucleus pulposus as determined by Iatridis $[30,31]$. Figure 5 shows that $\mathrm{G}^{*}$ and $\delta$ of N94, N94_2 and T94_2 do not vary much as a function of frequency. H94 on the other hand, clearly dissipates more energy upon increasing the frequency, which is in agreement with previous work [14]. 


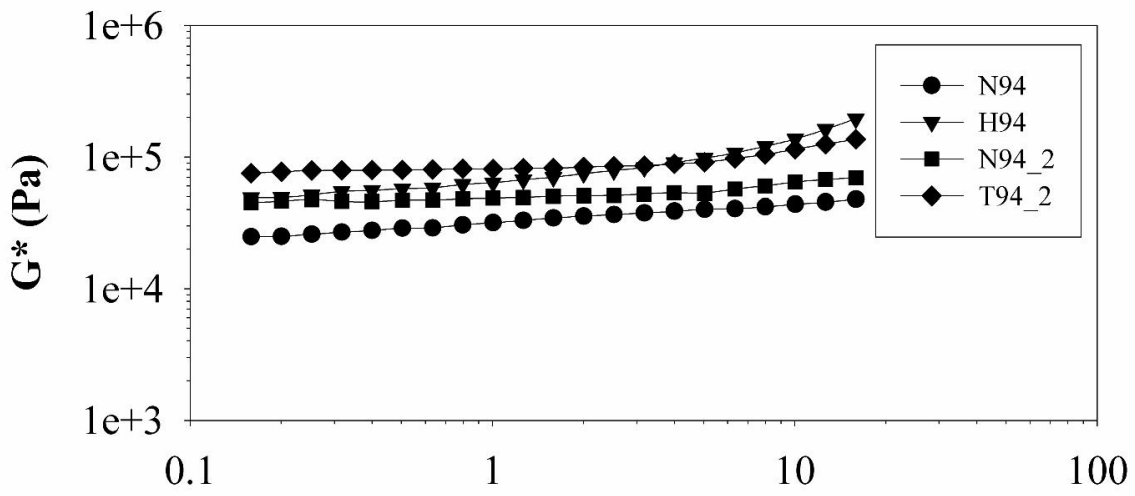

A

Frequency $(\mathrm{Hz})$

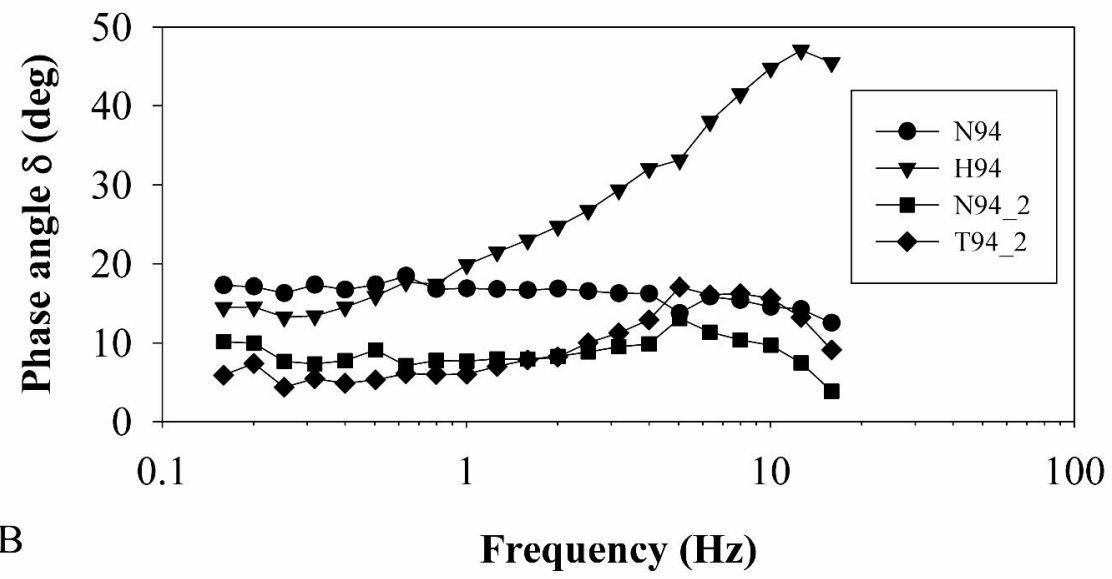

Figure 5: Rheological spectra from a dynamic shear experiment at $0.01 \%$ strain; $(A)$ complex shear modulus $G^{*},(B)$ phase angle $\delta$.

\subsection{Creep and fatigue}

To evaluate the ability of the materials to resist continuous loading and their recovery afterwards, a creep experiment was performed to an engineering stress of $0.1 \mathrm{MPa}$ [20], the basic physiologic stress the materials must be able to withstand without permanent deformation. The tests were conducted without radial constraint. The results are all significantly different and typical curves are 
depicted in Figure 6. Clearly, N94 displays a large creep effect, which causes it to deform beyond linearity (see Figure 4). The creep effect in H94 is smaller and this material recovers almost completely. The water content of H94 is low and the material is also somewhat crosslinked, which explains its elastic behaviour. Because of the significantly large creep effect of N94 and the low EWC of H94, both materials were not included in the fatigue test. Very clear is the influence of the crosslinker. Both N94_2 and T94_2 hardly show a creep effect and they both have practically no permanent deformation. The decrease in height directly after the test was $0 \%$ for both N94_2 and T94_2, 1\% for H94, and $7 \%$ for N94. The recovery of the hydrogels is in accordance with the phase angles given earlier. Those values already indicated that the crosslinked materials behave more elastically.

The materials were compressed to $15 \%$ in the fatigue experiment. Both N94_2 and T94_2 survive the million cycles without any apparent deterioration. Since the experiment was force controlled and radially unconstrained, the materials were seen to lose some height gradually during the test. The cumulative effect of the cyclic loading induces creep in the materials. The height loss was significantly more pronounced in N94_2 (95.3\% of height remaining), than in T94_2 (97.5\% of height remaining) at the end of the test.

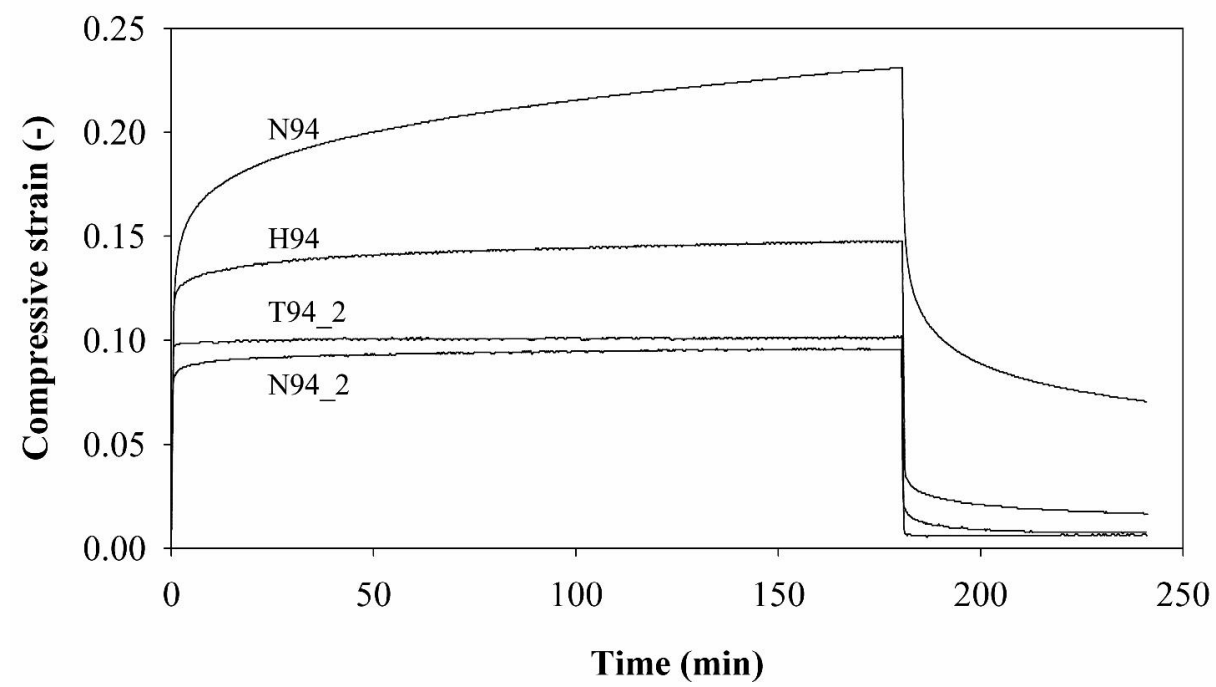

Figure 6: Creep behaviour and recovery of the hydrogels subjected to $0.1 \mathrm{MPa}$ for $3 h$. 


\section{Discussion}

Previous work on NVP/4IEMA copolymers revealed that leaching of NVP oligomers occurs [14]. This is an indirect consequence of the difference in reactivity of the monomers [32-34]. This reactivity difference causes 4IEMA to polymerize preferentially in the beginning of the polymerization reaction, and NVP towards the end, resulting in NVP oligomers. To reduce the amount of extractables, a crosslinker was used that crosslinks vinyl to methacrylate monomers. The commercially available monomer AMA has both an allyl group and a methacryl group [35] and was found to be a suitable crosslinker for our copolymers. After some trial experiments with different amounts of AMA, we decided to use 2 mol\% of AMA in the NVP/4IEMA copolymer, so N94 became N94_2. Obviously, the introduction of the crosslinker AMA significantly reduces mass loss, but also inevitably leads to a reduction in water content.

In analogy with the results of Lai et al. [35] we found that other crosslinkers, such as divinylbenzene and triethyleneglycol methacrylate a.o., are less suitable for crosslinking polymers based on NVP and methacrylates.

The material H94 does not show any mass loss in water, even without the addition of a chemical crosslinker. This is caused by physical and chemical crosslinking within the material [36-38].

Terpolymers of NVP, HEMA and 4IEMA were investigated as well, in an effort to combine the high water content of NVP-based copolymers with the elasticity of the HEMA-based copolymers in equilibrium with water at $37{ }^{\circ} \mathrm{C}$. The mass percentage of iodine in the final terpolymer was kept constant, while the ratio of NVP to HEMA was varied. These terpolymers also showed leaching of NVP oligomers, so they were also crosslinked. The combination of monomers shown in Table 1 as T94_2, seemed interesting in terms of water content and elasticity, so it was chosen to investigate this combination further.

The diffusion coefficient is used to estimate the time it takes for an implant of that material, with given dimensions, to reach equilibrium swelling. For an implant made out of T94_2 to swell to $1 \mathrm{~cm}$ lumbar disc height, the dry height will need to be $\sqrt[3]{Q}=1.26$ times smaller, so $0.79 \mathrm{~cm}$. The time to swell to equilibrium, will then be 103 hours. However, an implant does not have thin sheet geometry, so diffusion from the edges is also present and will cause the implant to reach equilibrium swelling quicker. In case of N94_2, the implant height would need to be $0.78 \mathrm{~cm}$ and swelling to equilibrium would take (less than) 62 hours. 
Regarding the mechanical characteristics, it should be noted that in situ, there is a synergistic interaction between the nucleus pulposus and the annulus fibrosus. This interaction is nearly impossible to replicate exactly in vitro. Introducing an artificial annulus will only introduce more unknown variables, hence it was chosen to perform the mechanical tests unconstrained. Since this poses an unrealistic challenge to the material, the main objective of the fatigue experiment was to see if the materials survive a million cycles, unconstrained. One million cycles was chosen as a preliminary indication of fatigue resistance. In a constrained situation, the materials are expected to perform even better. Another feature to mimic a more realistic situation would be the introduction of a resting period in the fatigue protocol to simulate sleep, in order to give the materials a chance to recover. This would also lead to improved performance. During unconfined compression the hydrogels display barrelling, which explains the deviation from linearity at large strains in the static experiment. The Young's modulus of a nucleus prosthesis needs to be in the range of $0.2-4$ $\mathrm{MPa}$ in order to mimic the mechanical behaviour of the natural nucleus pulposus [8, 39-41]. All materials are within this range. Périé et al. performed confined compression tests on bovine nucleus pulposus and found the modulus to be $0.31 \mathrm{MPa}$ [42].

Obviously, the natural nucleus is less stiff and has a more fluid consistency than our materials and also displays a high phase angle. This can be explained by the high water content of the nucleus. It is desirable for nucleus replacement materials to have a more solid consistency than the natural nucleus, so that they do not flow through the defect in the annulus fibrosus, as the natural nucleus does in case of HNP.

The natural disc creeps under loading, due to fluid flow [25]. During the day, the discs are continuously loaded and this introduces a creep effect, resulting in height loss. Overnight, the natural nuclei of the discs reswell and the disc heights are restored. This explains why on average people are $2 \mathrm{~cm}$ taller in the morning than in the afternoon [43]. To mimic this behaviour, we need a material that also recovers after loading.

\section{Concluding remarks}

The new crosslinked materials N94_2 and T94_2 were compared to N94 and H94. Already, the materials N94 and H94 showed to be good candidates for nucleus replacement [15], concerning implantability, biocompatibility and 
radiopacity, but they were improved further by incorporating the crosslinker AMA, without making concessions to these other properties. Introduction of this crosslinker led to a significant reduction in the number of extractables, improved elasticity and recovery after loading. Furthermore, the new materials exhibit good fatigue behaviour. Both N94_2 and T94_2 are expected to be able to cope with the strains occurring in the human intervertebral disc. They also combine considerable water uptake with excellent radio-visibility.

The high water content and absence of metallic markers makes the hydrogels easily detectable with MRI, whereas the covalently bound iodine renders them intrinsically radiopaque. While the spinal column is a very bony environment, already 5-6 mass\% of iodine is sufficient for visualizing the water-swollen materials in between two vertebrae.

Our results reveal that it is possible to design radiopaque hydrogel biomaterials that can replace the natural nucleus pulposus in terms of physical-mechanical properties and biocompatibility. CT or MRI imaging techniques can be used to evaluate the entire implant post-implantation. The next step in this research will be to evaluate the tissue response and long term mechanical behaviour of the prosthesis in vivo, in the nucleus cavity of an appropriate animal model. We envisage that especially young patients, who suffer from HNP or early DDD, can benefit from this strategy. This is a relatively small, but important subgroup of back pain patients.

\section{Acknowledgements}

This research was funded by the Dutch Polymer Institute (DPI), project number 398, Intervertebral Disc Prosthesis. The authors would like to thank professor Sjoerd Bulstra (Dept. of Orthopaedic Surgery, University Medical Center Groningen, the Netherlands) and dr. André van Ooij (Dept. of Orthopaedic Surgery, Academic Hospital Maastricht, the Netherlands) for constructive and stimulating discussions.

\section{References}

1. Andersson GB. Epidemiological features of chronic low-back pain. Lancet 1999; 354: 581-585.

2. Koes BW, van Tulder MW, Thomas S. Diagnosis and treatment of low back pain. Bmj 2006; 332: 1430-1434. 
3. Ihlebaek C, Eriksen HR. The "myths" of low back pain: status quo in norwegian general practitioners and physiotherapists. Spine 2004; 29: 1818-1822.

4. Bao QB, Yuan HA. New technologies in spine: nucleus replacement. Spine 2002; 27: $1245-1247$.

5. Guyer RD, Ohnmeiss DD. Intervertebral disc prostheses. Spine 2003; 28: S15-23.

6. Carl A, Ledet $E$, Yuan $H$, Sharan A. New developments in nucleus pulposus replacement technology. Spine J 2004; 4: 325S-329S.

7. Di Martino A, Vaccaro AR, Lee JY, Denaro V, Lim MR. Nucleus pulposus replacement: basic science and indications for clinical use. Spine 2005; 30: S1622.

8. Thomas J, Lowman A, Marcolongo M. Novel associated hydrogels for nucleus pulposus replacement. J Biomed Mater Res 2003; 67A: 1329-1337.

9. Husson JL, Korge A, Polard JL, Nydegger T, Kneubuhler S, Mayer HM. A memory coiling spiral as nucleus pulposus prosthesis: concept, specifications, bench testing, and first clinical results. J Spinal Disord Tech 2003; 16: 405-411.

10. Ray CD. The PDN prosthetic disc-nucleus device. Eur Spine J 2002; 11 Suppl 2: S137-142.

11. Bertagnoli R, Sabatino CT, Edwards JT, Gontarz GA, Prewett A, Parsons JR. Mechanical testing of a novel hydrogel nucleus replacement implant. Spine J 2005; 5: 672-681.

12. Buttermann GR, Beaubien BP. Stiffness of prosthetic nucleus determines stiffness of reconstructed lumbar calf disc. Spine J 2004; 4: 265-274.

13. Allen MJ, Schoonmaker JE, Bauer TW, Williams PF, Higham PA, Yuan HA. Preclinical evaluation of a poly (vinyl alcohol) hydrogel implant as a replacement for the nucleus pulposus. Spine 2004; 29: 515-523.

14. Boelen EJ, van Hooy-Corstjens CS, Bulstra SK, van Ooij A, van Rhijn LW, Koole LH. Intrinsically radiopaque hydrogels for nucleus pulposus replacement. Biomaterials 2005; 26: 6674-6683.

15. Boelen EJH, van Hooy-Corstjens CSJ, Gijbels MJJ, Bulstra SK, van Ooij A, van Rhijn LW, Koole LH. Preliminary evaluation of new intrinsically radiopaque hydrogels for replacing the nucleus pulposus. Journal of Materials Chemistry 2006; 16: 824-828.

16. Joshi A, Fussell G, Thomas J, Hsuan A, Lowman A, Karduna A, Vresilovic E, Marcolongo $M$. Functional compressive mechanics of a PVA/PVP nucleus pulposus replacement. Biomaterials 2006; 27: 176-184.

17. Benzina A, Kruft MAB, Bar F, van der Veen FH, Bastiaansen CW, Heijnen V, Reutelingsperger $\mathrm{C}$, Koole $\mathrm{LH}$. Studies on a new radiopaque polymeric biomaterial. Biomaterials 1994; 15: 1122-1128.

18. Khare AR, Peppas NA. Swelling/deswelling of anionic copolymer gels. Biomaterials 1995; 16: 559-567.

19. Shin H, Temenoff JS, Mikos AG. In vitro cytotoxicity of unsaturated oligo[poly(ethylene glycol) fumarate] macromers and their cross-linked hydrogels. Biomacromolecules 2003; 4: 552-560. 
20. Urban JP, McMullin JF. Swelling pressure of the lumbar intervertebral discs: influence of age, spinal level, composition, and degeneration. Spine 1988; 13: 179-187.

21. White AA, Panjabi MM. Clinical biomechanics of the spine. Philadelphia: Lippincott; 1978.

22. Crank J. The mathematics of diffusion. Oxford: Oxford University Press; 1990.

23. Bajpai AK, Shrivastava M. Water sorption dynamics of a binary copolymeric hydrogel of 2-hydroxyethyl methacrylate (HEMA). J Biomater Sci Polym Ed 2002; 13: 237-256.

24. Knetsch ML, Aldenhoff $\mathrm{YB}$, Hanssen $\mathrm{HH}$, Koole $\mathrm{LH}$. A novel synthetic vascular prosthesis: effect of plasma protein adsorption on blood - and cyto-compatibility. Mat.-wiss. u. Werkstofftech. 2006; 37: In press.

25. Ghosh P. The biology of the intervertebral disc. vol. 2. CRC Press; 1988.

26. Maroudas A, Stockwell RA, Nachemson A, Urban J. Factors involved in the nutrition of the human lumbar intervertebral disc: cellularity and diffusion of glucose in vitro. J Anat 1975; 120: 113-130.

27. Eijkelkamp MF, van Donkelaar CC, Veldhuizen AG, van Horn JR, Huyghe JM, Verkerke GJ. Requirements for an artificial intervertebral disc. Int J Artif Organs 2001; 24: 311-321.

28. Leahy JC, Hukins DW. Viscoelastic properties of the nucleus pulposus of the intervertebral disk in compression. J Mater Sci Mater Med 2001; 12: 689-692.

29. Reid JE, Meakin JR, Robins SP, Skakle JM, Hukins DW. Sheep lumbar intervertebral discs as models for human discs. Clin Biomech (Bristol, Avon) 2002; 17: $312-314$.

30. Iatridis JC, Weidenbaum M, Setton LA, Mow VC. Is the nucleus pulposus a solid or a fluid? Mechanical behaviors of the nucleus pulposus of the human intervertebral disc. Spine 1996; 21: 1174-1184.

31. Iatridis JC, Setton LA, Weidenbaum M, Mow VC. The viscoelastic behavior of the non-degenerate human lumbar nucleus pulposus in shear. J Biomech 1997; 30: 1005-1013.

32. Malak M, Hill DJT, Whittaker AK. Water sorption into poly[(2-hydroxyethyl methacrylate)-co-(1-vinyl-2-pyrrolidone)]at 310 K. Polymer International 2003; 52: $1740-1748$.

33. Atta AM, Arndt K-F. Swelling behavior of $\mathrm{pH}-$ and temperaturesensitive copolymers containing 2-hydroxyethyl methacrylate and $\mathrm{N}$-vinyl-2-pyrrolidone crosslinked with new crosslinkers. Polymer International 2004; 53: 1870-1881.

34. Davis TP, Huglin MB. Studies on Copolymeric Hydrogels of N-Vinyl-2-Pyrrolidone with 2-Hydroxyethyl Methacrylate. Macromolecules 1989; 22: 2824-2829.

35. Lai YC. Effect of Crosslinkers on Photocopolymerization of N-Vinylpyrrolidone and Methacrylates to Give Hydrogels. Journal of Applied Polymer Science 1997; 66: 1475-1489. 
36. Beers KL, Boo S, Gaynor SG, Matyjaszewski K. Atom transfer radical polymerization of 2-hydroxyethyl methacrylate. Macromolecules 1999; 32: 57725776.

37. Diakoumakos CD, Raptis I, Tserepi A, Argitis P. Free-radical synthesis of narrow polydispersed 2-hydroxyethyl methacrylate-based tetrapolymers for dilute aqueous base developable negative photoresists. Polymer 2002; 43: 1103-1113.

38. Refojo MF. Hydrophobic Interaction in Poly(2-hydroxyethyl Methacrylate) Homogeneous Hydrogel. Journal of Polymer Science; Part A-1 1967; 5: 31033113.

39. Meakin JR. Replacing the nucleus pulposus of the intervertebral disk: prediction of suitable properties of a replacement material using finite element analysis. J Mater Sci Mater Med 2001; 12: 207-213.

40. Meakin JR, Reid JE, Hukins DW. Replacing the nucleus pulposus of the intervertebral disc. Clin Biomech (Bristol, Avon) 2001; 16: 560-565.

41. Fagan MJ, Julian S, Siddall DJ, Mohsen AM. Patient-specific spine models. Part 1: Finite element analysis of the lumbar intervertebral disc--a material sensitivity study. Proc Inst Mech Eng [H] 2002; 216: 299-314.

42. Perie D, Korda D, Iatridis JC. Confined compression experiments on bovine nucleus pulposus and annulus fibrosus: sensitivity of the experiment in the determination of compressive modulus and hydraulic permeability. J Biomech 2005; 38: 2164-2171.

43. Roberts N, Hogg D, Whitehouse GH, Dangerfield P. Quantitative analysis of diurnal variation in volume and water content of lumbar intervertebral discs. Clin Anat 1998; 11: 1-8. 
90 | Chapter 4 


\section{Evaluation of a Highly Radiopaque Iodine- Containing Acrylic Bone Cement for Use in Augmentation of Vertebral Compression Fractures}

Erik J.H. Boelen, Gladius Lewis, Jie Xu, Tristan Slots, Leo H. Koole, Catharina S.J. van Hooy-Corstjens

Adapted from:

Journal of Biomedical Materials Research. Part A. Submitted 


\section{Abstract}

Vertebroplasty and balloon kyphoplasty are widely used for the augmentation of osteoporosis-induced vertebral compression fractures. Almost invariably, an injectable poly (methyl methacrylate) (PMMA) bone cement that contains a large amount of $\mathrm{BaSO}_{4}$ particles is used in these procedures. The deleterious effects of this radiopacifier on various properties of PMMA cement have been detailed in the literature. The objective of the present study was therefore to avoid such high levels of inorganic contrast agent and thus to develop an all-polymeric bone cement, for which radiopacity was provided by $60 \mathrm{wt} \%$ of an iodinecontaining methacrylic copolymer, incorporated into the powder (IO cement), ultimately leading to $6.6 \mathrm{wt} . \%$ of iodine in the cement. A large array of properties of this cement were determined, ranging from setting time and injectability to fatigue life under fully-reversed tension-compression loading and cytotoxicity, and a comprehensive comparison with a cement containing 30 wt. $\% \mathrm{BaSO}_{4}$ in the powder component (BA cement) has been made (11 wt.\% of $\mathrm{Ba}$ in the cement). Statistical analyses of the results showed that, for the majority of the properties, the difference between the means for the two cements was not significant. It is therefore suggested that the IO cement is a suitable alternative to the BA cement for use in the aforementioned procedures. 


\section{Introduction}

Symptomatic compression fractures of vertebral bodies that occur secondary to osteoporosis are a major public health issue. (For example, the annual direct costs of these fractures are estimated to be $\sim \$ 750$ million and $\$ 440$ million in the US and in the European Union countries, respectively [1, 2]). Vertebroplasty (VP) and balloon kyphoplasty (KP) are minimally-invasive surgical procedures that are rapidly becoming popular modalities for the stand-alone augmentation of these fractures, especially those that have proved to be refractory to conservative methods and/or medical therapy [3, 4]. (An estimated 38,000 VPs and $16,000 \mathrm{KPs}$ were performed in the US in 2002 [5]). Each of these procedures involves the percutaneous delivery of a bolus of an injectable bone cement into the fractured vertebral body, carried out under very careful fluoroscopic image guidance [6]. Because of its long history of successful use in cemented total joint replacements (CTJRs), poly (methyl methacrylate) (PMMA) bone cements are used in most VPs and KPs. Whereas contemporary acrylic bone cements for CTJR contain 10-15 wt.\% of radiopacifier in the powder phase, cements for VP and KP require a higher radiopacifier content for improved visualization under X-ray fluoroscopy. This is achieved by using either a commercially-available brand that is formulated specifically for such use (for example, KyphX ${ }^{\circledR} \mathrm{HV}-\mathrm{RTM}$, which contains $30 \mathrm{wt} . \% \mathrm{BaSO}_{4}$, and Osteopal ${ }^{\circledR} \mathrm{V}$, which contains $33 \mathrm{wt} \% \mathrm{ZrO}_{2}$ particles) or one that is traditionally used in CTJRs (for example, Surgical Simplex ${ }^{\circledR} \mathrm{P}$, which contains $10 \mathrm{wt} . \% \mathrm{BaSO}_{4}$ particles) but with extra amounts (typically, 6-10 g) of $\mathrm{BaSO}_{4}$ or $\mathrm{ZrO}_{2}$ particles and/or $1-2 \mathrm{~g}$ of tantalum or tungsten powder hand mixed, by the surgeon/nurse, into the cement powder just prior to injection [6-10].

Concerns have been raised about this practice. In the case of $\mathrm{BaSO}_{4}$ particles, it has been shown that (i) increased concentration leads to decreases in a number of quasi-static mechanical properties of a cement that are relevant to its use in VP and KP, such as fracture toughness and flexural strength [11, 12], as well as in fatigue life [13]; and (ii) they tend to form clumps in the powder which, in turn, act as fatigue crack initiation sites [14]. Furthermore, in skeletally mature sheep, foreign-body giant cells were observed, at 90 days, around $\mathrm{BaSO}_{4}$ particles in the case when a PMMA cement containing $30 \mathrm{wt} . \%$ $\mathrm{BaSO}_{4}$ was used whereas few such particles were observed, after the same period, when a cement containing $10 \mathrm{wt} . \% \mathrm{BaSO}_{4}$ was used [15]. $\mathrm{ZrO}_{2}$ particles are very hard and not compatible with the polymer matrix [16]. Both $\mathrm{BaSO}_{4}$ and 
$\mathrm{ZrO}_{2}$ particles have been shown to enhance macrophage-osteoclast differentiation, which may contribute to bone resorption, which is particularly undesirable in osteoporotic persons [17]. The influence of $W$ or Ta powders on the cement properties is unknown [18].

To avoid using high levels of inorganic contrast agents, we developed an allpolymeric radiopaque bone cement. Radiopacity was introduced by incorporating an iodine-containing methacrylic copolymer into the cement powder. This copolymer was prepared as a $1 / 1 \mathrm{w} / \mathrm{w}$ copolymerization of methyl methacrylate (MMA) with a methacrylic monomer containing covalently bound iodine (2-[4'-iodobenzoyl]-oxo-ethyl methacrylate, 4IEMA) [19]. There are a number of articles in the literature about iodine-containing acrylic bone cements, with the iodine present in either the liquid monomer $[20,21]$ or, as in our approach, in the powder [19, 22-24]. However, all of these cements were developed for use in CTJRs, implying that the radiopacity will be too low for VP and KP. In our previous studies, it was shown that a bone cement with low amounts of an iodine-containing methacrylic copolymer (3.5 wt.\% iodine) present in the powder component of the cement, displayed good fatigue performance and excellent biocompatibility [19, 23, 24]. Building on these results, we have prepared an acrylic bone cement for which the content of iodine-containing copolymer (I-copolymer) has been increased to an iodine content of 10.6 wt.\% of the powder. Thus, this new cement (herein called the IO cement) is intrinsically radiopaque at a level that is deemed acceptable for VP and KP.

The present work is a comprehensive comparison of the IO cement and a 30 wt. $\% \mathrm{BaSO}_{4}$-containing PMMA bone cement (BA cement) on the basis of a wide collection of in vitro physical, handling, thermal, mechanical, and cytotoxic characteristics. Even though these properties include a sizeable proportion of those that have been suggested as being important for an injectable bone cement to be used in VP and KP $[6,11]$, the comparison between BA and IO cements is limited by two facts. First, there are no standards for characterizing injectable bone cements for use in VP and KP. Second, there are only a very few acrylic bone cement brands that are formulated specifically for use in VP and KP and, for these cements, there are no data for the large majority of the properties determined in the present work. The present study should therefore be regarded as a contribution to the development of (i) a new generation of injectable acrylic bone cements for use in VP and KP, and (ii) standards for characterizing these cements. 


\section{Materials and Methods}

\subsection{Materials}

To make a fair comparison between the BA and IO cement, all components, except the radiopacifier, should be the same. Therefore, all experiments were performed with own-made cement, based on own-made PMMA microspheres. These microspheres were prepared via a suspension polymerization technique, using benzoyl peroxide (BPO), as the initiator. Some of the initiator remained in these microspheres after completion of the reaction, with the residual BPO content being determined, by titration, to be $2.27 \mathrm{wt} . \%$. The I-copolymer (copolymer of MMA and 4IEMA (mass ratio 1/1)), which was used as the radiopacifier for the IO cement, was also prepared via a suspension polymerization reaction. The resulting microspheres had a residual BPO content of $1.11 \mathrm{wt} . \%$. The compositions of both cements are given in Table I. For VP and KP, liquid monomer-to-powder ratio's (LPR) between 0.22 and $1.43 \mathrm{~mL} \mathrm{~g}^{-1}$ have been described in literature [7-10]. In the present study, we used a LPR of $0.65 \mathrm{~mL} \mathrm{~g}^{-1}$.

Table 1: Compositions ${ }^{a}$ of the cements

\begin{tabular}{|c|c|c|c|c|c|c|c|}
\hline \multirow{2}{*}{ Cement } & \multicolumn{4}{|c|}{ Powder (g) } & \multicolumn{3}{|c|}{ Liquid monomer (g) } \\
\hline & PMMA & $\mathrm{BaSO}_{4}$ & $\begin{array}{l}\text { I- } \\
\text { copolymer }\end{array}$ & BPO & MMA & DMPT & HQ \\
\hline $\begin{array}{l}\text { BA } \\
\text { cement }\end{array}$ & 27.36 & 12.0 & 0.0 & 0.64 & 23.4 & 0.6 & $20^{b}$ \\
\hline $\begin{array}{l}\text { IO } \\
\text { cement }\end{array}$ & 15.64 & 0.0 & 23.73 & 0.63 & 23.4 & 0.6 & $20^{b}$ \\
\hline \multicolumn{8}{|c|}{$\begin{array}{l}\text { 'PMMA: poly (methyl methacrylate); MMA: methyl methacrylate; I-copolymer: } \\
\text { copolymer of MMA and 2-[4'-iodobenzoyl]-oxo-ethyl methacrylate }(1 / 1 \mathrm{w} / \mathrm{w}) \text {; } \\
\text { BPO: benzoyl peroxide; DMPT: } N, N \text {-dimethyl- } p \text {-toluidine; HQ: hydroquinone. } \\
\text { 'In ppm. }\end{array}$} \\
\hline
\end{tabular}




\subsection{Characterization of powders}

The particle size distributions of both the PMMA spheres and I-copolymer spheres were determined by measuring the diameters of 500 microspheres using light microscopy. The morphologies of the cement powders were obtained using a scanning electron microscope (XL30; Philips, Eindhoven, The Netherlands) operating at an accelerating voltage of $15 \mathrm{kV}$.

\subsection{Preparation and morphology of as-cured cements}

For the determination of cement properties, the powder and the liquid monomer were mixed using an open-bowl technique (hand mixing), under ambient laboratory conditions (temperature and relative humidity of $20 \pm 1{ }^{\circ} \mathrm{C}$ and $55 \pm 2 \%$, respectively), as is commonly done in VP and KP [7-10]. The morphologies of the as-cured cements were obtained using machined disc specimens (diameter and height of $10 \mathrm{~mm}$ and $1 \mathrm{~mm}$, respectively) and a scanning electron microscope (XL30; Philips Eindhoven, The Netherlands) operating at an accelerating voltage of $10 \mathrm{kV}$ in the backscatter mode.

\subsection{Evaluation of radiopacity}

A cured cement disc (diameter and thickness of $12 \mathrm{~mm}$ and $3 \mathrm{~mm}$, respectively) was irradiated with X-rays (Philips BV Pulsera; $46 \mathrm{kV}, 1.04 \mathrm{mAs}$, autofocus), much as is done in clinical practice. Following a method that has been described in the literature [25], a commercially-available image editing software (Adobe ${ }^{\circledR}$ Photoshop ${ }^{\circledR}$; Adobe, Inc., San Jose, CA) was used to measure the brightness of the disc in the resulting image, averaged over an area of 5 pixels $\times 5$ pixels. The radiopacity of the cement was then determined as the ratio of its darkness ( $=100$ - brightness) to that of aluminum (thickness $3 \mathrm{~mm}$ ), measured using the same procedure. Also the radiopacity of a $10 \mathrm{wt} . \% \mathrm{BaSO}_{4}$ cement for CTJR was determined for comparison. Measurements were made in six-fold.

\subsection{Injectability tests}

These were carried out using a method proposed by other authors [26, 27]. 1 min after mixing the cement, the dough was charged into a $20-\mathrm{mL}$ disposable syringe that was attached to a bone biopsy needle (diameter and length of 1.6 
$\mathrm{mm}$ and $150 \mathrm{~mm}$, respectively). Through a universal materials testing machine, a 1-kg compressive load was applied to the top of the syringe's plunger. Injectability was defined as the mass of cement dough extruded through the needle into a poly (tetrafluoroethylene) beaker, over a period of $1 \mathrm{~min}$, as a proportion of the mass of cement charged into the syringe. Six measurements were made for each cement.

\subsection{Residual monomer content}

The residual monomer content (RMC) of the fully cured cements was determined using proton nuclear magnetic resonance ( $\left.{ }^{1} \mathrm{H}-\mathrm{NMR}\right)$ spectroscopy (Varian Mercury 400; Varian Corp., Palo Alto, CA, USA), operating at $400 \mathrm{MHz}$, with tetramethylsilane as the internal standard. Approximately $50 \mathrm{mg}$ of the cured cement was accurately weighed at different time points, $t$, after curing ( $t$ $=1$ day, 1 week, 1 year) and dissolved in $2.5 \mathrm{~mL}$ deuterated acetone. $\mathrm{A}$ solution of $1 \mathrm{mg} \mathrm{mL}^{-1} \mathrm{MMA}$ in deuterated acetone was also prepared. For each combination of cement and $\mathrm{t}, 400 \mu \mathrm{L}$ of the cement solution, together with 0 , 100,200 or $300 \mu \mathrm{L}$ of the MMA solution was transferred to an NMR-tube and completed to $700 \mu \mathrm{L}$ using pure deuterated acetone. The NMR spectrum of the mixture was then recorded. The vinyl proton signals in the spectrum (singlets at 5.6 and $6.0 \mathrm{ppm}$ ) (Figure $1 \mathrm{~A}$ ) were integrated and set to 100 arbitrary units for the cement solution. This integral, together with those when the mixture contained cement solution and known added amounts of MMA (100, 200, 300 $\mu \mathrm{g}$ ), were plotted and the best-fit trend line was obtained (Figure 1B). The intersection of this trendline with the $x$-axis represents the amount of MMA present in the cement solution. With this value, the percentage of residual monomer in the dissolved cement was calculated. 


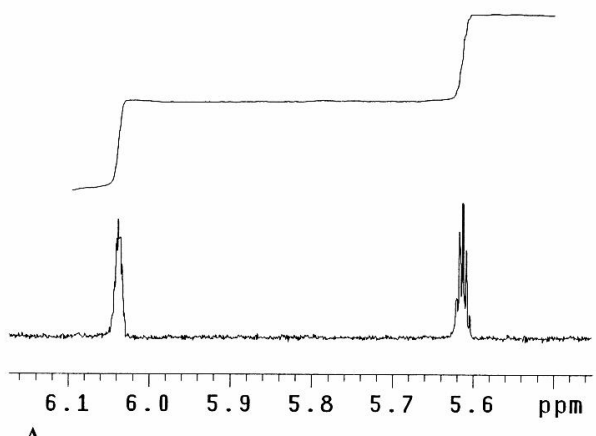

A

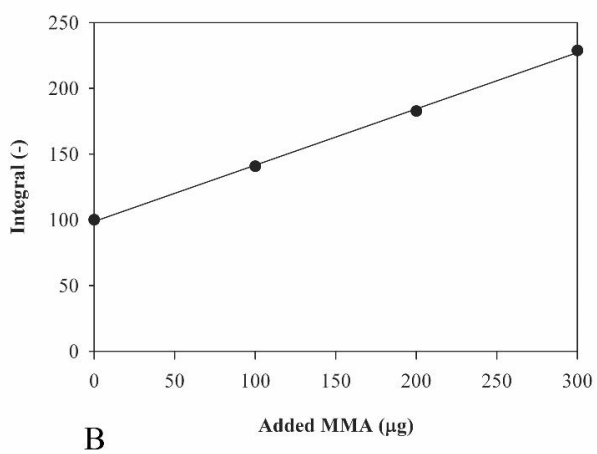

B

Figure 1: (A) Expanded view ( $\delta=5.5-6.1 \mathrm{ppm}$ ) of a typical $400 \mathrm{MHz}{ }^{1} \mathrm{H}-\mathrm{NMR}$ spectrum of acrylic bone cement, showing the vinyl proton signals of residual $M M A$ and $(B)$ typical trendline-curve for the determination of the residual monomer content, using ${ }^{1} H-N M R$.

\subsection{PBS uptake}

The gain in the mass of a circular cross-sectioned cement disc specimen (diameter and length of 2.80 and $8.00 \mathrm{~mm}$, respectively), which was immersed

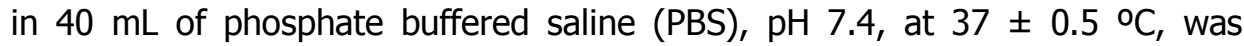
monitored continuously until there was no significant increase; that is, process equilibrium was reached.

The early stages of the uptake $\left(\mathrm{M}_{\mathrm{t}} / \mathrm{M}_{\infty}<0.6\right)$, are describable by a reduced form of an applicable solution to Fick's Second Law of Diffusion (Stefan's approximation), which is [28]:

$$
\frac{M_{t}}{M_{\infty}}=4\left(\frac{D t}{\pi l^{2}}\right)^{0.5}
$$

where $M_{t}$ and $M_{\infty}$ are the mass gains of the specimen after time, $t$, in the PBS solution, and at the equilibrium stage, respectively. Thus, $D$ (the diffusion coefficient) was computed from the slope of the linear plot of $M_{t} / M_{\infty}$ versus $\sqrt{ } t$. For each cement, three specimens were tested.

\subsection{Handling properties}

The setting time $\left(t_{s e t}\right)$ and the maximum exotherm temperature $\left(T_{\max }\right)$ were determined, in ambient laboratory air $\left(20 \pm 1^{\circ} \mathrm{C}\right)$. The liquid monomer and 
powder were hand mixed with a spatula, $25 \mathrm{~g}$ of the cement mixture was insulated in aluminium foil, and a thermometer was inserted in the middle of the dough. The temperature was recorded every $10 \mathrm{~s}$ until polymerization was complete. For each cement, the test was run in duplicate.

\subsection{Bulk quasi-static mechanical properties}

The compressive yield strength (YS) and compressive modulus (E) were determined using machined solid cylindrical test specimens (diameter and height of both $6 \mathrm{~mm}$ ) and a servohydraulically-actuated universal materials testing machine (MTS 810 Elastomer Test System; MTS, Eden Prairie, MN, USA) operated at a true strain rate of $3 \times 10^{-3} \mathrm{~s}^{-1}$. YS is defined as the stress at the point of permanent yield, zero slope, on the stress-strain curve. Before testing, the samples were incubated in water at $37^{\circ} \mathrm{C}$ till equilibrium was reached. For each cement, six specimens were tested.

The plane-strain fracture toughness ( $\mathrm{K}_{\mathrm{IC}}$ ) of each cement was determined using ASTM D 5045 compact tension fracture toughness $\left(\mathrm{K}_{\mathrm{IC}}\right)$ specimens [29] having width, thickness, and initial crack length of 37.17, 14.87, and $14.87 \mathrm{~mm}$, respectively. The moulded specimens were cured for $2 \mathrm{~h}$, after which they were removed from the mould, lightly sanded, and then conditioned in PBS, at $37^{\circ} \mathrm{C}$, for $28 \mathrm{~d}$. The $\mathrm{K}_{\mathrm{IC}}$ tests were carried out, in ambient laboratory air, using a servohydraulically-actuated universal materials testing machine (Bionix 810; MTS, Eden Prairie, MN, USA), under displacement control, with a cross-head speed of $10 \mathrm{~mm} \mathrm{~min}$. All other test procedures and data analysis methods followed were as given in ASTM D 5045. For each cement, six specimens were tested.

\subsection{Quasi-static nanoindentation properties}

Moulded specimens ( $45 \mathrm{~mm} \times 12 \mathrm{~mm} \times 5 \mathrm{~mm}$ bars) were stored in PBS, at 37 ${ }^{\circ} \mathrm{C}$ until they reached equilibrium mass (this took $23 \pm 1 \mathrm{~d}$ ), before the tests were performed using a commercially-available nanoindentation instrument (TriboIndenter ${ }^{\circledR}$; Hysitron, Inc., Minneapolis, MN, USA).

The indenter was driven into the surface of the specimen at a constant loading rate of $30 \mu \mathrm{N} \mathrm{s}^{-1}$ until a peak load of $260 \mu \mathrm{N}$ was reached. The values of the hardness, $\mathrm{H}_{\mathrm{N}}$, and elastic modulus, $\mathrm{E}_{\mathrm{N}}$, were calculated using the Modified Slopes Method, MSM [30]. Further details about (i) specimen preparation, the performance of the test, data acquisition, and the treatment of the data using 
MSM to obtain $\mathrm{H}_{\mathrm{N}}$ and $\mathrm{E}_{\mathrm{N}}$ for acrylic bone cement specimens; (ii) the reason for using MSM, rather than the well known Oliver-Pharr method; and (iii) validation of the test have all been given previously [31]. Three specimens per cement were tested.

\subsection{Fatigue performance}

The test specimen configuration and size, specimen preparation, specimen examination and, hence, selection for testing, specimen conditioning, and test procedures were all as detailed in ASTMF 2118-03 [32]. Stress levels (S) of \pm $10 \mathrm{MPa}, \pm 15 \mathrm{MPa}$, and $\pm 20 \mathrm{MPa}$ were used. The rejection rates (number of specimens discarded after inspection, as detailed in the standard, as a proportion of the total number of specimens moulded) were $40 \%$ and $45 \%$ for BA cement and IO cement specimens, respectively, values that are within the range reported in the literature [33]. For each combination of cement and $S$ of $\pm 20 \mathrm{MPa}$ or $\pm 15 \mathrm{MPa}, 12$ specimens selected at random from the accepted specimens were tested, consistent with the recommendation of Lewis and Sadhasivini [34]. (For $\mathrm{S}$ of $\pm 10 \mathrm{MPa}, 8$ specimens were used for each cement because of time constraints - some specimens experienced "run-out"; that is, no fatigue failure after 1.5 million stress cycles).

The cycles to fracture, $N_{f}$, results were used in the estimation of the fatigue limit of the cement through the use of the Olgive equation [35]:

$S=A+(B-A) /\left(1+\left(\ln N_{f} / C\right) D\right)$,

where $A, B, C$, and $D$ are material constants.

(In this case, the parameter of interest was $A$, which was taken to be the "fatigue limit" of the cement). A commercially-available software (Matlab ${ }^{\circledR}$ Version 7.0, The MathWorks, Natick, MA, USA) was used in the curve-fitting exercise.

For each cement, the morphology of the fracture surfaces of the fatigue specimens, at $\mathrm{S}= \pm 15 \mathrm{MPa}$, was obtained using a scanning electron microscope (Model XL30; Philips, Eindhoven, the Netherlands), operating at an accelerating voltage of $15 \mathrm{kV}$. (This value of $\mathrm{S}$ was chosen because, of the three values of $S$ used, it is the lowest value for which all the specimens in both sets fractured). 


\subsection{Dynamic nanomechanical properties}

Moulded specimens ( $45 \mathrm{~mm} \times 12 \mathrm{~mm} \times 5 \mathrm{~mm}$ bars) were stored in PBS, at 37 ${ }^{\circ} \mathrm{C}$ until they reached equilibrium mass (this took $23 \pm 1 \mathrm{~d}$ ) before the tests were performed using a commercially-available nanoindentation instrument (TriboIndenter ${ }^{\circledR}$ ).

The indenter was driven, under computer control, toward the surface of the specimen, at a constant rate of $10 \mathrm{~nm} \mathrm{~s}^{-1}$, until it contacted the surface. Upon contact, a small, constant oscillatory load of $15 \mu \mathrm{N}$, at a selected value of $\omega$ (1$200 \mathrm{~Hz}$, in steps of $\sim 7 \mathrm{~Hz}$ ), superimposed on a quasi-static load of $200 \mu \mathrm{N}$, was applied to the surface. The values of the storage modulus $\left(E^{\prime}\right)$ and loss modulus $\left(E^{\prime \prime}\right)$, at a given value of $\omega$ were determined assuming that the head assembly of the instrument may be modelled as a simple damped harmonic oscillator [36]. Further details of (i) specimen preparation, performance of the test, data acquisition, and the treatment of the data using the aforementioned model to obtain $\mathrm{E}^{\prime}$. and $\mathrm{E}^{\prime \prime}$ for acrylic bone cement specimens; (ii) validation of the test; and (iii) the limitations of the experimental method used have all been given previously [37]. Three specimens per cement were tested.

\subsection{Cytotoxicity tests}

Small fragments (total mass of approx. $0.25 \mathrm{~g}$ ) of the cured cements, exposed to ambient laboratory air for 2 months were sterilized using ultra-violet light and then put in approx. $2.5 \mathrm{~mL}$ (final concentration $0.1 \mathrm{~g} \mathrm{~mL}^{-1}$ ) cell culture medium (Minimum essential medium with Earle's salts, Glutamax-I, and $25 \mathrm{mM}$ HEPES and supplemented with $10 \mathrm{wt} \%$ fetal bovine serum and antibiotic/antimycotic solution $(1 \times))$ at $37^{\circ} \mathrm{C}$. The medium was removed after 2 $\mathrm{d}$, stored at $4^{\circ} \mathrm{C}$, and fresh medium was added. This was repeated twice. The collected media were put on osteoblast cells (SAOS-2). After $3 \mathrm{~d}$, a methyl thiazol tetrazolium (MTT) assay [38] was conducted and the cell survival was calculated by measuring the optical absorbance at $550 \mathrm{~nm}$. The cell survival in pure culture medium was set to $100 \%$. All tests were run in triplicate.

\subsection{Statistical analysis}

The results for the cement properties were statistically analyzed using ANOVA, with the Bonferroni correction, and the $95 \%$ confidence limits for the 
differences between population means (SAS ${ }^{\circledR}$ Version 8.02; SAS Institute Inc., Cary, NC, USA).

\section{Results}

\subsection{Powder characteristics and morphology of as- cured cements}

The number average particle diameter of the PMMA and I-copolymer spheres was found to be $15.4 \pm 13.2 \mu \mathrm{m}$ and $14.9 \pm 12.9 \mu \mathrm{m}$ respectively, whereas the weight average particle diameter was $86.6 \pm 72.5 \mu \mathrm{m}$ and $89.9 \pm 76.2 \mu \mathrm{m}$ respectively. The size distribution diagrams can be seen in Figure 2 . Clearly, there is no significant difference between the sizes of the PMMA and Icopolymer spheres. So, the main difference between the cement powders is the presence of $\mathrm{BaSO}_{4}$ particles in the $\mathrm{BA}$ cement powder, which can clearly be seen in the SEM images (Figure 3).

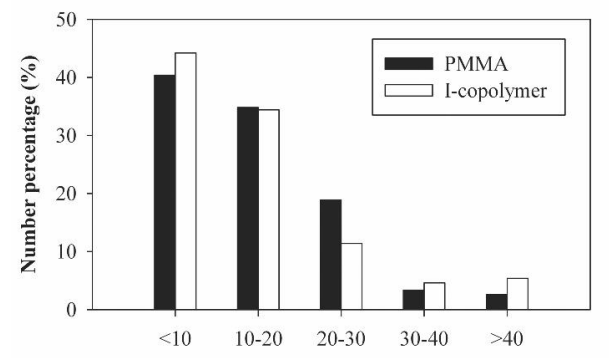

A

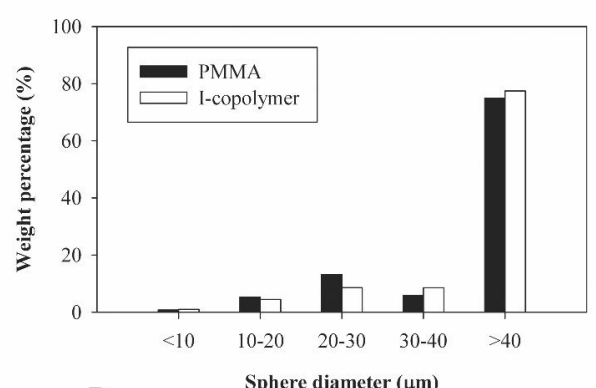

B

Figure 2: Size distribution diagrams of PMMA and I-copolymer microspheres; (A) number-weighted distribution diagram and (B) mass-weighted distribution diagram. 


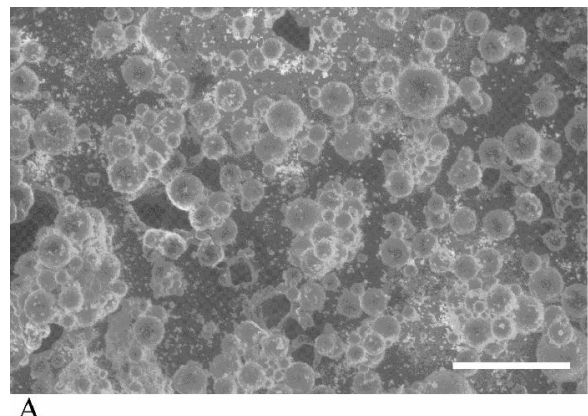

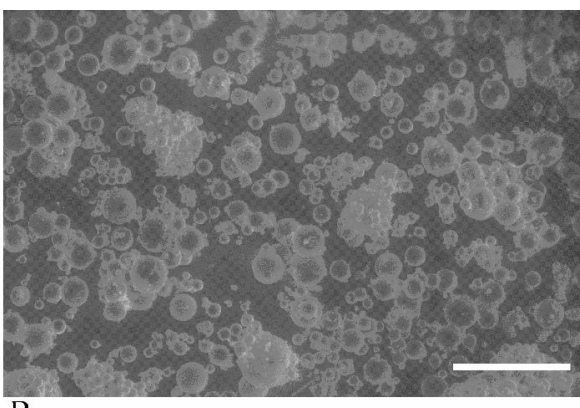

$\mathrm{B}$

Figure 3: Scanning electron micrographs of the powder of (A) BA cement and (B) IO cement. Mark that, in the $B A$ cement, the $\mathrm{BaSO}_{4}$ particles are seen as white clusters. (Scale bar is $100 \mu \mathrm{m}$ ).

The radiopacifier in the BA powder is seen as discrete white clusters (Figure $3 \mathrm{~A})$, while it is indistinguishable as such from the IO powder (Figure 3B), which is to be expected because the radiopacity is incorporated in $60 \%$ of the spheres. The different radiopacifiers in the two cements are more clearly seen in the backscatter images of the as-cured cements (Figure 4). In the BA cement (Figure $4 \mathrm{~A}$ ), $\mathrm{BaSO}_{4}$ particles and lumps (white) are clearly visible between the black PMMA microspheres. In the IO cement (Figure 4B), there are light spheres (I-copolymer) and dark spheres (PMMA microspheres). Also, the surrounding matrix in the IO cement is somewhat lighter, due to the presence of the I-copolymer in the matrix, as a result of the partial dissolution of the Icopolymer in the matrix during mixing of the liquid monomer and the powder. The penetration of the MMA into the I-copolymer spheres during the mixing is apparent from the darkened corona of the light spheres.

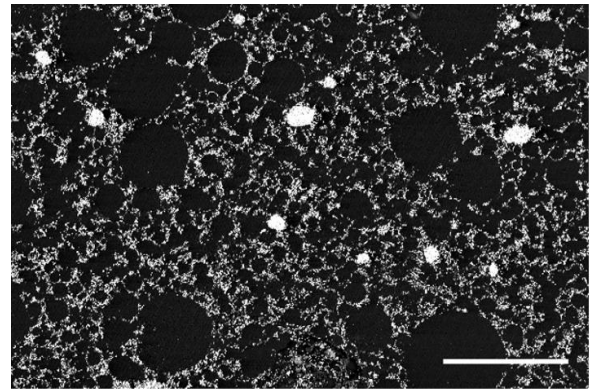

A

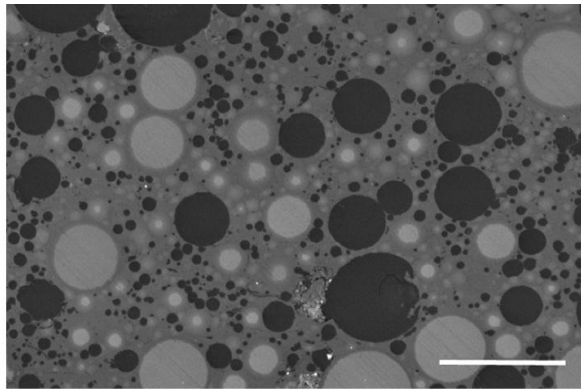

$\mathrm{B}$

Figure 4: Backscatter scanning electron micrographs of the as-cured cements, clearly showing the difference in radiopacifier between $(A) B A$ cement and (B) IO cement. (Scale bar is $200 \mu \mathrm{m}$ ). 


\subsection{Physical, handling, polymerization, and bulk mechanical properties}

The values of all these properties for the BA and IO cements are presented in Table II.

The radiopacity of both cements exceeds that of aluminium of the same thickness (108.2\% for IO cement and $132.9 \%$ for BA cement) and it is also considerably higher than that of acrylic bone cement used in CTJRs, which was found to be average $66 \%$. The difference between the cements was found to be significant (Figure 5 and Table III).

When mixing the cements, a clear difference in rheology was observed between them, with IO cement flowing more easily than BA cement during the very early stage of the test. This difference is not reflected in the injectability results, which show no significant difference between the cements (Table III).

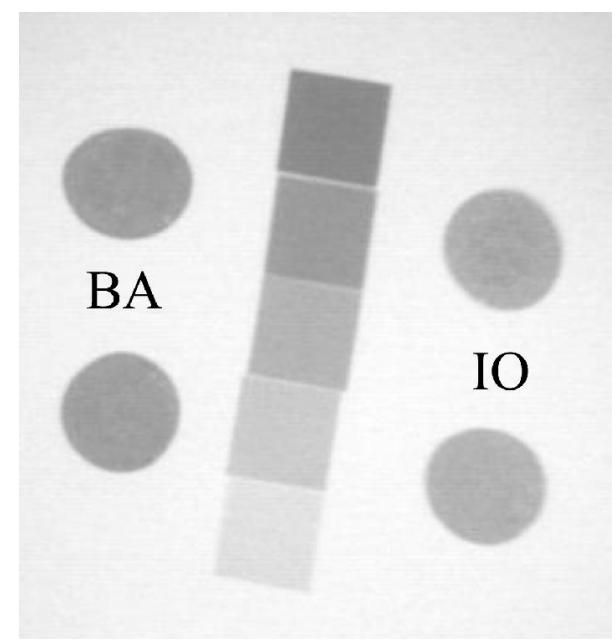

Figure 5: Radiograph of $3 \mathrm{~mm}$ thick discs of the cements and of an aluminium step wedge (1 to $5 \mathrm{~mm}$ thick, $1 \mathrm{~mm}$ steps).

For both cements, as expected, the RMC decreases markedly with increase in time after curing, and, after $1 \mathrm{y}$, the RMCs of the two cements are essentially the same $(<1 \%$, Table II).

The fact that there is no significant difference in the diffusion coefficients for the uptake of PBS by the two cements points to the possibility that the uptake mechanism - infiltration of the fluid into the micro-voids or molecular interaction of available hydrogen bonds at the hydrophilic sites [39] - is the same for both cements. 
The IO cement polymerizes significantly faster but with a significantly lower maximum exotherm temperature than the BA cement (Table III). Although the residual BPO content in the I-copolymer microspheres was adjusted to ensure that the total amount of BPO in the powder phase was equal in both cements, the availability of BPO is different. Since IO cement contains more microspheres (100\% microspheres in the powder of the IO cement versus $70 \%$ microspheres in the powder of the BA cement), the surface area for MMA to diffuse into the spheres is larger. This explains the faster polymerization of IO cement. (Note that, in both cements, all the BPO is present in the form of residual BPO in the microspheres and no free BPO is added). During VP or KP, the injectable bone cement bolus is introduced percutaneously into the body of the fractured vertebra, so a lower $T_{\max }$ is desirable to avoid excessive thermal necrosis [40]. Mechanically, there is no significant difference in either the YS or the $\mathrm{K}_{\mathrm{IC}}$ values for the two cements. Furthermore, for both $H_{N}$ and $E_{N}$, the difference in the means for the two cements is not significant (Table III).

The fatigue limits were estimated to be $10.4 \mathrm{MPa}$ (95\% confidence limits: $8.3-$ $12.5 \mathrm{MPa}$ ) for IO cement and $10.0 \mathrm{MPa}$ (95\% confidence limits of $8.1-11.8$ $\mathrm{MPa}$ ) for BA cement (Figure 6).

However, there are two features of the fatigue limit estimation results that need comment. First, the computed curve does not approach a lower asymptotic stress value, which should be the fatigue limit. Second, some specimens suffered fatigue at $\pm 10 \mathrm{MPa}$. In other words, the estimation of the fatigue limit using the Olgive equation is problematic for these cements, a situation that may be a consequence of the extensive overlap of the $\mathrm{N}_{\mathrm{f}}$ results obtained at \pm 20 $\mathrm{MPa}$ and $\pm 15 \mathrm{MPa}$. In light of these points, it may be prudent to adopt the lower $95 \%$ confidence level estimates as the fatigue limits. Also, there is a marked difference in the morphologies of the fracture surfaces of the BA and IO cements (Figure 7), with the BA cement fracture surface comprising some sectioned PMMA microspheres and pores that were most likely the sites of crack initiation, and the IO cement fracture surface being characterized by smooth, almost glass-like, stacked plateaux. More important than the relative values of the estimated fatigue limits of the two cements is the fact that both estimates are within the range that the present authors computed for $\mathrm{KyphX}^{\circledR} \mathrm{HVR}$ cement, which is a high-radiopacity PMMA cement that is currently used in VP and KP. (This estimate, 9.3 MPa, with 95\% confidence intervals of 7.0 and 11.5 $\mathrm{MPa}$, was obtained from the fit of the tension-compression fatigue life results given by Kurtz et al. [13] to Equation (2)). 

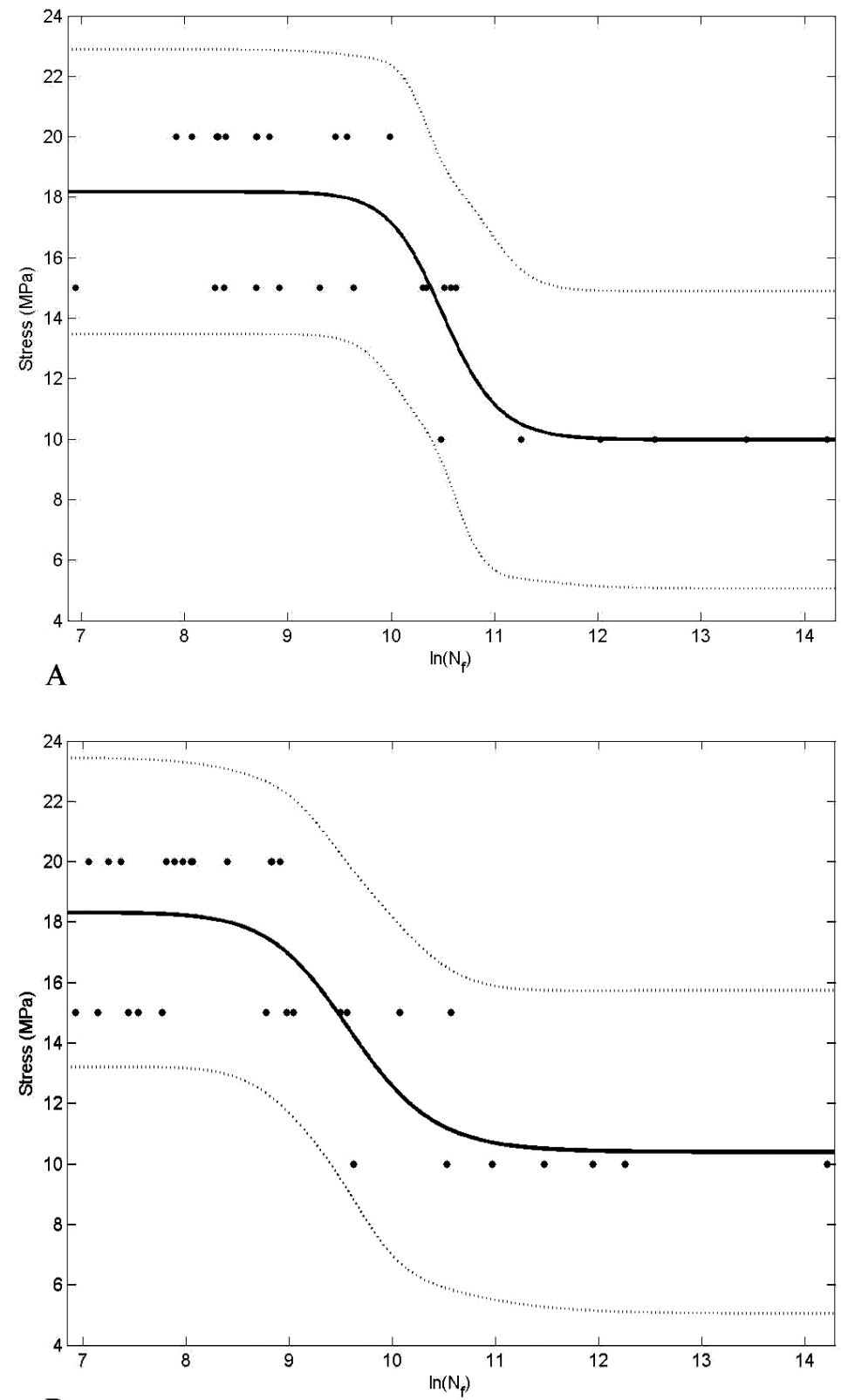

B

Figure 6: The fatigue life $\left(N_{f}\right)$ results and fits to the Olgive equation with the associated $95 \%$ confidence intervals for (A) BA cement and (B) IO cement. 


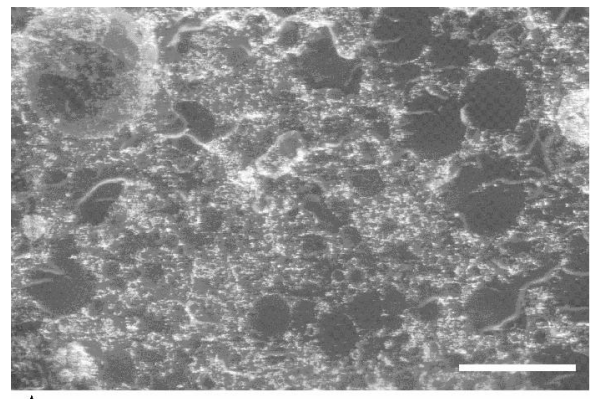

A

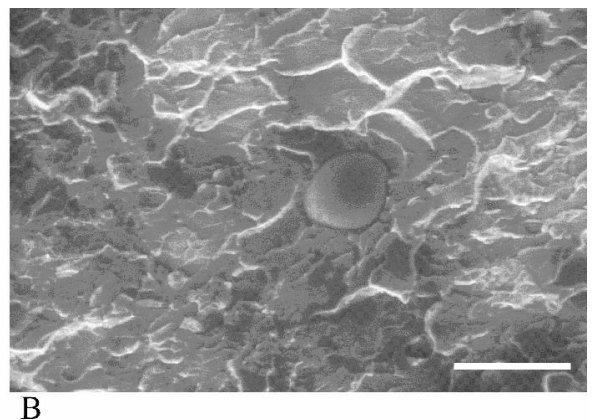

B

Figure 7: The morphologies of the fracture surfaces of representative fatigue specimens of (A) BA cement and (B) IO cement. (Scale bar is $100 \mu \mathrm{m}$ ).

The results of the dynamic nanoindentation tests (Figure 8) showed that the difference in the viscoelastic behaviour of the cements was not significant. For example, (i) the $E^{\prime}$ values range from $3.85 \pm 0.74 \mathrm{GPa}$ at $1 \mathrm{~Hz}$ to $5.52 \pm 1.00$ $\mathrm{GPa}$ at $200 \mathrm{~Hz}$ for the BA cement and from $3.74 \pm 0.43 \mathrm{GPa}$ at $1 \mathrm{~Hz}$ to $5.28 \pm$ $0.50 \mathrm{GPa}$ at $200 \mathrm{~Hz}$ for the IO cement; and (ii) the minimum value of $\mathrm{E}^{\prime \prime}(\sim 0)$ occurs at an applied frequency of $\sim 83 \mathrm{~Hz}$ and $\sim 77 \mathrm{~Hz}$ for BA and IO cements, respectively.

\subsection{Cytotoxicity performance}

In the cytotoxicity test, the first extract of both cements appeared to be toxic to the osteoblast cells, since cell survival was low $(<1 \%)$ (Table II). The second extract of the IO cement was found to be considerably less toxic than that of the BA cement. Apparently less toxic substances dissolve from the IO cement. This difference could not be seen anymore in the third extract. 


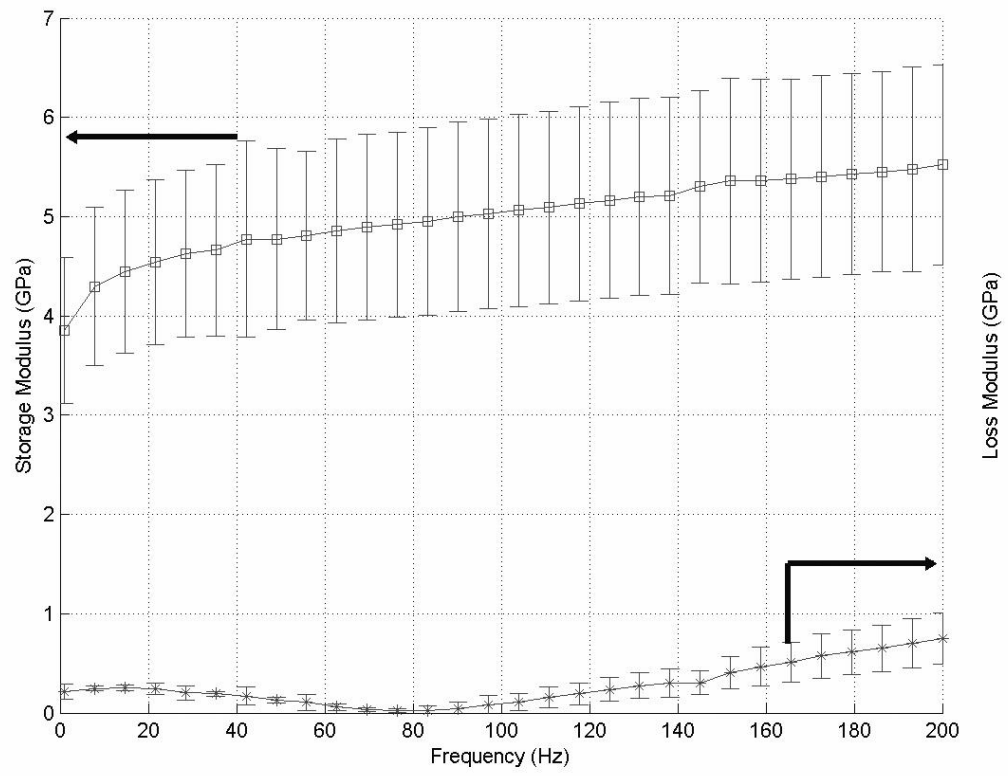

A

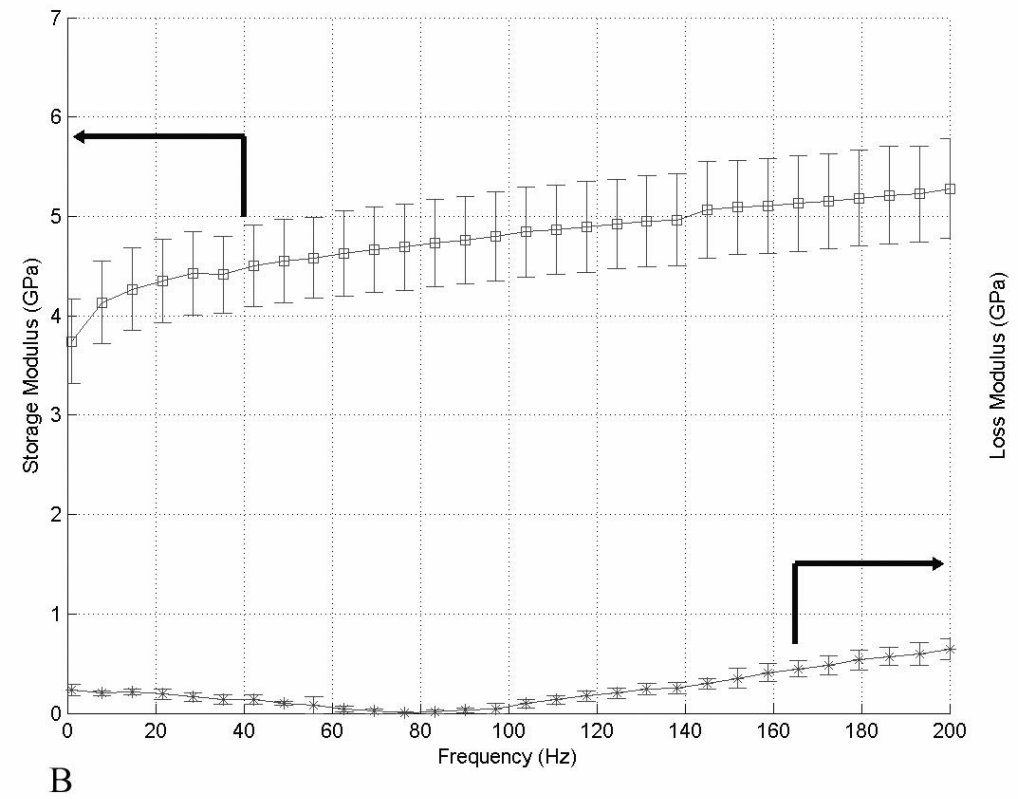

Figure 8: Typical results of the variation of the storage modulus and the loss modulus with frequency of the applied load, obtained in the dynamic nanoindentation tests for (A) BA cement and (B) IO cement. 
Table 2: Summary of the values of the properties of the cements.

\begin{tabular}{|c|c|c|}
\hline Property & BA cement & IO cement \\
\hline Radiopacity (\% of aluminum) & $132.9 \pm 3.6$ & $108.2 \pm 8.3$ \\
\hline Injectability (\%) & $88.3 \pm 2.2$ & $85.8 \pm 3.1$ \\
\hline $\begin{array}{l}\text { Residual monomer content ( } 1 \text { day; } \\
1 \text { week; } 1 \text { year) }(\%)\end{array}$ & $5.3 ; 2.4 ; 0.2$ & $8.4 ; 2.9 ; 0.9$ \\
\hline $\begin{array}{l}\text { Diffusion coefficient, } D \times 10^{12}\left(\mathrm{~m}^{2}\right. \\
\left.\mathrm{s}^{-1}\right)\end{array}$ & $4.26 \pm 0.75$ & $3.66 \pm 0.59$ \\
\hline Setting time, $\mathbf{t}_{\text {set }}(\mathrm{min})$ & $18.5 \pm 0$ & $12.5 \pm 0$ \\
\hline $\begin{array}{l}\text { Maximum exotherm temperature, } \\
T_{\max }\left({ }^{\circ} \mathrm{C}\right)\end{array}$ & $101 \pm 0.7$ & $94 \pm 0.7$ \\
\hline $\begin{array}{l}\text { Compressive yield strength, YS } \\
\text { (MPa) }\end{array}$ & $60.5 \pm 3.9$ & $57.6 \pm 5.9$ \\
\hline Compressive modulus, E (MPa) & $1610 \pm 130$ & $1736 \pm 40$ \\
\hline Fracture toughness, $K_{\mathrm{IC}}(\mathrm{MPa} \sqrt{\mathrm{m}})$ & $1.61 \pm 0.05$ & $1.58 \pm 0.07$ \\
\hline Fatigue limit (MPa) & 10.0 & 10.4 \\
\hline Hardness, $\mathrm{H}_{\mathrm{N}}(\mathrm{MPa})$ & $204 \pm 11$ & $210 \pm 16$ \\
\hline Elastic modulus, $\mathrm{E}_{\mathrm{N}}(\mathrm{GPa})$ & $3.67 \pm 0.26$ & $3.73 \pm 0.24$ \\
\hline $\begin{array}{l}\text { Cytotoxicity ( } \% \text { cell survival of } 1^{\text {st }} \text {, } \\
2^{\text {nd }} \text {, and } 3^{\text {rd }} \text { extract) }\end{array}$ & $\begin{array}{l}0.3 \pm 0.1 ; 20.8 \pm \\
4.3 ; 72.1 \pm 8.5\end{array}$ & $\begin{array}{l}0.6 \pm 0.1 ; 58.9 \\
\pm 9.1 ; 70.6+ \\
8.1\end{array}$ \\
\hline
\end{tabular}


Table 3: Results of the ANOVA, with Bonferroni post hoc, on results of IO cement versus those of $B A$ cement.

\begin{tabular}{|c|c|c|c|}
\hline Property & $\begin{array}{l}\text { Difference } \\
\text { between } \\
\text { means } \\
\end{array}$ & $\begin{array}{l}\text { Simultaneous } \\
95 \% \text { confidence } \\
\text { limits }\end{array}$ & Outcome $^{a}$ \\
\hline $\begin{array}{l}\text { Radiopacity（\% } \\
\text { of Al) }\end{array}$ & -24.70 & $-32.93 ;-16.47$ & $S$ \\
\hline $\begin{array}{l}\text { Injectability } \\
(\%)\end{array}$ & -2.50 & $-5.96 ; 0.96$ & NS \\
\hline $\begin{array}{l}D \times 10^{12}\left(\mathrm{~m}^{2}\right. \\
\left.\mathrm{s}^{-1}\right)\end{array}$ & -0.60 & $-2.13 ; 0.93$ & NS \\
\hline$t_{\text {set }}(\mathrm{min})$ & -6.00 & $-6.00 ;-6.00$ & $S$ \\
\hline $\mathbf{T}_{\max }\left({ }^{\circ} \mathrm{C}\right)$ & -7.00 & $-10.01 ;-3.99$ & $S$ \\
\hline YS (MPa) & -2.90 & $-24.42 ; 18.62$ & NS \\
\hline E (MPa) & 126.00 & $2.28 ; 249.72$ & $S$ \\
\hline$K_{\text {IC }}(\mathrm{MPa} \sqrt{ } \mathrm{m})$ & -0.03 & $-0.11 ; 0.05$ & NS \\
\hline $\mathrm{H}_{\mathrm{N}}(\mathrm{MPa})$ & 6.00 & $-25.12 ; 37.12$ & NS \\
\hline$E_{N}(G P a)$ & 0.06 & $-0.51 ; 0.63$ & NS \\
\hline $\begin{array}{l}\text { Cytotoxicity } 1^{\text {st }} \\
\text { extract }\end{array}$ & 0.30 & $0.21 ; 0.39$ & $S$ \\
\hline $\begin{array}{l}\text { Cytotoxicity } 2^{\text {nd }} \\
\text { extract }\end{array}$ & 38.10 & $31.41 ; 44.79$ & $\mathrm{~S}$ \\
\hline $\begin{array}{l}\text { Cytotoxicity } 3^{\text {rd }} \\
\text { extract }\end{array}$ & -1.50 & $-9.30 ; 6.30$ & NS \\
\hline
\end{tabular}

as: difference between means is significant; NS: difference between means is not significant

\section{Discussion}

There is a growing literature on the characterization of highly-radiopaque acrylic bone cements that are used or are proposed for use in VP and KP $[13,26,41$ 50]. However, (i) in each of these studies, the cement used contained an 
inorganic material as the radiopacifier; (ii) in some studies, only a few properties were determined [41-47, 49, 50]; (iii) in vitro and in vivo biocompatibility of the cements was determined in only two studies [47, 48]; (iv) the uptake of a physiological salt solution $\left(0.9 \% \mathrm{NaCl}\right.$, at $\left.37{ }^{\circ} \mathrm{C}\right)$ was the subject of one study [47]; and (v) residual monomer content was determined in only one study [26]. In contrast, in the present study, (i) for one of the cements, we utilized an innovative approach to produce high radiopacity without using any inorganic materials; and (ii) all the aforementioned properties (except the in-vivo biocompatibility), together with an array of other relevant ones, were determined. Thus, the present study is the most comprehensive to date.

There are no standards for the determination of any properties (and, hence, there are no property limits) of highly-radiopaque acrylic bone cements for use in VP and KP. However, the present injectablity, RMC, $t_{\text {set, }} T_{\max }$, and $Y S$ results are within the ranges reported for other high-radiopacity acrylic cements that are being used in VP and $\mathrm{KP}[13,41,42,50]$ or have been suggested as having the potential for such use $[26,48]$.

In the present study, the fatigue performance under fully-reversed tensioncompression loading was determined. This, arguably, represents the most aggressive type of alternating loading. Soltesz et al. [51] found that, for two acrylic bone cements that are widely used in CTJRs, fatigue strength at $10^{7}$ cycles (which may be taken as the fatigue limit), under fully-reversed tensioncompression load is only $8-18 \%$ and $15-34 \%$ that under cyclic 4-point bend and cyclic tension-tension, respectively. Thus, the fatigue limits estimated for BA and IO cements may be regarded as being conservative. Intradiscal pressure measurements at the L4-L5 level, made with a telemetric system, suggest that the compressive stresses on lumbar vertebrae during normal activities of daily living range from 0.1 $\mathrm{MPa}$ to 2.3 $\mathrm{MPa}$ [52]. Thus, given the fatigue limits estimated for BA and IO cements ( 8-12 MPa), it is unlikely that a fractured vertebral body augmented using VP or KP and either of these cements will suffer fatigue failure.

Clinically, the final curing of the bolus occurs at a temperature closer to $37^{\circ} \mathrm{C}$ than to the operating room temperature (typically, $21^{\circ} \mathrm{C}$ ). It is known that $\mathrm{t}_{\text {set }}$ and $T_{\max }$ of an acrylic bone cement decreases and increases, respectively, with increase in measurement temperature [11]. Another point of consideration is that $T_{\max }$ is largely influenced by the amount of cement used, due to an autoacceleration effect of the polymerization reaction. These factors make it very difficult to relate the observed values to actual setting times and maximum exotherm temperatures that will be obtained in the clinical setting. (No 
comments can be made regarding the acceptability of these "clinically-relevant" values because there are no stated limits for $t_{\text {set }}$ and $T_{\max }$ for acrylic bone cements for VP and KP).

In VP and KP, the injected cement bolus is required to mechanically interlock with the bone. Thus, strictly speaking, in the determination of the in vitro mechanical properties of the cement, a technique that characterizes the outermost layers of a cement specimen should be used; that is, the technique should allow determination of property measures at the microstructural scale at or near the surface. It was for this reason that the nanoindentation technique was used in the present study to obtain both quasi-static and dynamic mechanical properties. (The displacement noise floor or displacement sensitivity, of a nanoindentation instrument is, typically, between 0.1 and 0.3 $\mathrm{nm})$. Only few reports are available on $\mathrm{H}_{\mathrm{N}}$ and $\mathrm{E}_{\mathrm{N}}$ of acrylic bone cements and these are for the low-radiopacity brands [31, 37]. There are a number of literature studies on $\mathrm{E}^{\prime}$. and. $\mathrm{E}^{\prime \prime}$ of low-radiopacity acrylic bone cements, as a function of test temperature, at a fixed value of $\omega$ (in most cases, $1 \mathrm{~Hz}$ ) [20, $53,54]$, but very few on $E^{\prime}$. and $E^{\prime \prime}$ as a function of $\omega$, at a fixed temperature $[37,55]$, with nanoindentation used in only one case [37].

It has been shown that an iodine-containing acrylic bone cement that differs from the one used in the present study only in the amount of the iodinecontaining copolymer incorporated into the powder ( $20 \mathrm{wt} . \%$ versus $60 \mathrm{wt} \%$ in the present study) is biocompatible [24]. This finding, coupled with the results of the present MTT assays, leads us to believe that IO cement is also biocompatible.

IO cement is in the development phase and, so, there is scope for modifying its properties through, for example, optimizing its composition and the LPR used; for example, (i) increasing the BPO content will lead to a decrease in $t_{\text {set }}$ but $\mathrm{T}_{\max }$ may increase [11]; and (ii) decreasing the LPR may lead to decreases in both $t_{\text {set }}$ and $T_{\max }$ [11]. (Clinicians will find information regarding the influence of LPR on $t_{\text {set }}$ and $T_{\max }$ particularly helpful). It must also be remembered that the iodine content of the I-copolymer and the amount of I-copolymer in the IO cement (which determine the cement's radiopacity) were selected on an empirical basis (for both cements the fraction of radiopacifier in the powder component has been increased by a factor 3 compared to cements for CTJRs), with the expectation that the cement's radiopacity will be about the same as that for the BA cement. In other words, the radiopacity of the IO cement can easily be increased by increasing the iodine content from its current level of $10.6 \mathrm{wt} \%$. (Of course, the extent to which this increase will affect other properties of the curing and cured cement will need to be investigated). 
Further characterization of both BA and IO cements should include obtaining (i) the fatigue limit of the cements, under tension-compression loading using a more direct and time-saving method, such as thermography [56]; (ii) cement injection data, such as syringe pressure-versus-injection time $(T)$ and injected cement volume-versus-T profiles; (iii) cement resorption and osteoconductivity patterns; and (iv) the compressive fatigue lives of osteoporotic cadaveric vertebral bodies, augmented using the cement, while immersed in an appropriate biosimulating solution, at $37^{\circ} \mathrm{C}$. Clearly, all the aforementioned properties and those determined in the present study are important in characterizing the cements. However, it is stressed that the ultimate comparison of the cements can only be obtained from well-designed, randomized, controlled clinical trials of VP and KP in which the subjects in the study groups are matched (for age, sex, weight, and numbers) and all the variables, notably, level(s) augmented, number of levels augmented, surgical technique (unipedicular versus bipedicular approach), and volume of cement injected, are the same for the study groups except for the cement used.

\section{Conclusions}

A large collection of properties were determined for two highly-radiopaque acrylic bone cements that have the same composition but differ only in the radiopacifier used; $30 \mathrm{wt} \% \mathrm{BaSO}_{4}$ particles (BA cement) or $60 \mathrm{wt} \%$ of an iodine-containing copolymer incorporated in the powder (IO cement). For a majority of these properties, the difference in the means was not significant. Given the concerns that have been raised about $\mathrm{BaSO}_{4}$ particles in acrylic bone cement, the present results suggest that IO cement is a viable alternative to high $\mathrm{BaSO}_{4}$-containing cement in procedures for augmenting osteoporosisinduced vertebral compression fractures, such as vertebroplasty and balloon kyphoplasty.

\section{Acknowledgements}

This research was funded by the Dutch Polymer Institute (DPI). The authors thank Dr. Sied Janna, and Ms. Naga Pallavi Neti, for their contributions to the fracture toughness, and fatigue testing. 


\section{References}

1. Melton LJ, 3rd. Epidemiology of spinal osteoporosis. Spine 1997; 22: 2S-11S.

2. Johnell O. Economic implication of osteoporotic spine disease: cost to society. Eur Spine J 2003; 12 Suppl 2: S168-169.

3. Carrino JA, Chan R, Vaccaro AR. Vertebral augmentation: vertebroplasty and kyphoplasty. Semin Roentgenol 2004; 39: 68-84.

4. Hadjipavlou AG, Tzermiadianos MN, Katonis PG, Szpalski M. Percutaneous vertebroplasty and balloon kyphoplasty for the treatment of osteoporotic vertebral compression fractures and osteolytic tumours. J Bone Joint Surg $\mathrm{Br}$ 2005; 87: 1595-1604.

5. Nussbaum DA, Gailloud P, Murphy K. A review of complications associated with vertebroplasty and kyphoplasty as reported to the Food and Drug Administration medical device related web site. J Vasc Interv Radiol 2004; 15: 1185-1192.

6. Lewis G. Injectable bone cements for use in vertebroplasty and kyphoplasty: state-of-the-art review. J Biomed Mater Res B Appl Biomater 2006; 76: 456-468.

7. Theodorou DJ, Theodorou SJ, Duncan TD, Garfin SR, Wong WH. Percutaneous balloon kyphoplasty for the correction of spinal deformity in painful vertebral body compression fractures. Clin Imaging 2002; 26: 1-5.

8. Vasconcelos C, Gailloud P, Beauchamp NJ, Heck DV, Murphy KJ. Is percutaneous vertebroplasty without pretreatment venography safe? Evaluation of 205 consecutives procedures. AJNR Am J Neuroradiol 2002; 23: 913-917.

9. Jensen ME, Evans AJ, Mathis JM, Kallmes DF, Cloft HJ, Dion JE. Percutaneous polymethylmethacrylate vertebroplasty in the treatment of osteoporotic vertebral body compression fractures: technical aspects. AJNR Am J Neuroradiol 1997; 18: 1897-1904.

10. Grados F, Depriester C, Cayrolle G, Hardy N, Deramond H, Fardellone P. Longterm observations of vertebral osteoporotic fractures treated by percutaneous vertebroplasty. Rheumatology (Oxford) 2000; 39: 1410-1414.

11. Nussbaum DA, Gailloud P, Murphy K. The chemistry of acrylic bone cements and implications for clinical use in image-guided therapy. J Vasc Interv Radiol 2004; 15: 121-126.

12. May B, Saha S. The effect of increased barium sulphate on the mechanical properties of bone cement. In: Transactions of the 28th Annual Meeting of the Society for Biomaterials; 2002; Tampa, FL, USA. 390.

13. Kurtz SM, Villarraga ML, Zhao K, Edidin AA. Static and fatigue mechanical behavior of bone cement with elevated barium sulfate content for treatment of vertebral compression fractures. Biomaterials 2005; 26: 3699-3712.

14. Bhambri SK, Gilbertson LN. Micromechanisms of fatigue crack initiation and propagation in bone cements. J Biomed Mater Res 1995; 29: 233-237.

15. Kobayashi N, Togawa D, Fujishiro T, Powell KA, Turner AS, Seim HB, 3rd, Bauer TW. Histological and radiographic evaluation of polymethylmethacrylate with two 
different concentrations of barium sulfate in a sheep vertebroplasty model. J Biomed Mater Res A 2005; 75: 123-127.

16. Kasperk C, Hillmeier J, Noldge G, Grafe IA, Dafonseca K, Raupp D, Bardenheuer $\mathrm{H}$, Libicher M, Liegibel UM, Sommer U, Hilscher U, Pyerin W, Vetter M, Meinzer HP, Meeder PJ, Taylor RS, Nawroth P. Treatment of painful vertebral fractures by kyphoplasty in patients with primary osteoporosis: a prospective nonrandomized controlled study. J Bone Miner Res 2005; 20: 604-612.

17. Sabokbar A, Fujikawa Y, Murray DW, Athanasou NA. Radio-opaque agents in bone cement increase bone resorption. J Bone Joint Surg Br 1997; 79: 129-134.

18. Persson C, Guandalini L, Baruffaldi F, Pierotti L, Baleani M. Radiopacity of tantalum-loaded acrylic bone cement. Proc Inst Mech Eng [H] 2006; 220: 787791.

19. van Hooy-Corstjens CS, Govaert LE, Spoelstra AB, Bulstra SK, Wetzels GM, Koole LH. Mechanical behaviour of a new acrylic radiopaque iodine-containing bone cement. Biomaterials 2004; 25: 2657-2667.

20. Artola A, Goni I, Gil J, Ginebra P, Manero JM, Gurruchaga M. A radiopaque polymeric matrix for acrylic bone cements. J Biomed Mater Res B Appl Biomater 2003; 64: 44-55.

21. Artola A, Gurruchaga M, Vazquez B, San Roman J, Goni I. Elimination of barium sulphate from acrylic bone cements. Use of two iodine-containing monomers. Biomaterials 2003; 24: 4071-4080.

22. Wang JS, Diaz J, Sabokbar A, Athanasou N, Kjellson F, Tanner KE, McCarthy ID, Lidgren $\mathrm{L}$. In vitro and in vivo biological responses to a novel radiopacifying agent for bone cement. J R Soc Interface 2005; 2: 71-78.

23. Lewis G, van Hooy-Corstjens CS, Bhattaram A, Koole LH. Influence of the radiopacifier in an acrylic bone cement on its mechanical, thermal, and physical properties: barium sulfate-containing cement versus iodine-containing cement. J Biomed Mater Res B Appl Biomater 2005; 73: 77-87.

24. van Hooy-Corstjens CS, Bulstra SK, Knetsch ML, Geusens P, Kuijer R, Koole LH. Biocompatibility of a new radiopaque iodine-containing acrylic bone cement. J Biomed Mater Res B Appl Biomater 2006.

25. Kjellson F, Almen T, Tanner KE, McCarthy ID, Lidgren L. Bone cement X-ray contrast media: a clinically relevant method of measuring their efficacy. J Biomed Mater Res B Appl Biomater 2004; 70: 354-361.

26. Carrodeguas RG, Lasa BV, Del Barrio JS. Injectable acrylic bone cements for vertebroplasty with improved properties. J Biomed Mater Res B Appl Biomater 2004; 68: 94-104.

27. Gbureck U, Spatz K, Thull R, Barralet JE. Rheological enhancement of mechanically activated alpha-tricalcium phosphate cements. J Biomed Mater Res B Appl Biomater 2005; 73: 1-6.

28. Vergnaud JM. Liquid transport processes in polymeric materials. Upper Saddle River, NJ, USA: Prentice-Hall; 1991. 
29. ASTM. Standard D 5045-99. Standard test methods for plain-strain fracture toughness and strain energy release rate of plastic materials. In: Annual Book of ASTM Standards, vol. 08.02. West Conshohocken, PA, USA: ASTM International; 2005: 800-808.

30. Bull SJ. Extracting hardness and Young's modulus from load-displacement curve. Zeitschrift Fur Metallkunde 2002; 93: 870-874.

31. Lewis G, Xu J, Dunne N, Daly C, Orr J. Critical comparison of two methods for the determination of nanomechanical properties of a material: application to synthetic and natural biomaterials. J Biomed Mater Res B Appl Biomater 2006; 78: 312317.

32. ASTM. Standard F 2118-03. Standard test method for constant amplitude of force controlled fatigue testing of acrylic bone cement materials. In: Annual Book of ASTM Standards, vol. 13.01. West Conshohocken, PA, USA: ASTM International; 2005: 1182-1189.

33. Prendergast PJ, Murphy BP, Taylor D. Letter to the editor. Fatigue Fract Eng Mater Struct 2002; 25: 315-316.

34. Lewis G, Sadhasivini A. Estimation of the minimum number of test specimens for fatigue testing of acrylic bone cement. Biomaterials 2004; 25: 4425-4432.

35. Krause W, Mathis RS, Grimes LW. Fatigue properties of acrylic bone cement: S-N, P-N, and P-S-N data. J Biomed Mater Res 1988; 22: 221-244.

36. Loubet JL, Oliver WC, Lucas BN. Measurement of the loss tangent of low-density polyethylene with a nanoindentation technique. Journal of Materials Research 2000; 15: 1195-1198.

37. Lewis G, Xu J, Dunne N, Daly C, Orr J. Evaluation of an accelerated aging medium for acrylic bone cement based on analysis of nanoindentation measurements on laboratory-prepared and retrieved specimens. J Biomed Mater Res B Appl Biomater 2006.

38. Mosmann T. Rapid colorimetric assay for cellular growth and survival: application to proliferation and cytotoxicity assays. J Immunol Methods 1983; 65: 55-63.

39. Bellenger V, Verdu J, Morel E. Structure-Properties Relationships for Densely Cross-Linked Epoxide Amine Systems Based on Epoxide or Amine Mixtures .2. Water-Absorption and Diffusion. Journal of Materials Science 1989; 24: 63-68.

40. Belkoff SM, Molloy S. Temperature measurement during polymerization of polymethylmethacrylate cement used for vertebroplasty. Spine 2003; 28: 15551559.

41. Jasper LE, Deramond H, Mathis JM, Belkoff SM. Material properties of various cements for use with vertebroplasty. J Mater Sci Mater Med 2002; 13: 1-5.

42. Brunella MF, Puncioni A, Cigada A. Effect of the preparation technique on dynamic mechanical behavior of acrylic cements for vertebroplasty. J Appl Biomater Biomech 2004; 2: 197.

43. Baroud G, Matsushita C, Samara M, Beckman L, Steffen T. Influence of oscillatory mixing on the injectability of three acrylic and two calcium-phosphate bone 
cements for vertebroplasty. J Biomed Mater Res B Appl Biomater 2004; 68: 105111.

44. Baroud G, Samara M, Steffen T. Influence of mixing method on the cement temperature-mixing time history and doughing time of three acrylic cements for vertebroplasty. J Biomed Mater Res B Appl Biomater 2004; 68: 112-116.

45. Perry A, Mahar A, Massie J, Lu L, Yaszemski MJ, Garfin SR, Kim CW. Development and biomechanical evaluation of an injectable radiopaque polypropylene fumarate bone cement for kyphoplasty. In: Transactions of the 30th Annual Meeting of the Society for Biomaterials; 2005; Memphis, TN, USA. 568.

46. Hernandez L, Gurruchaga M, Goni I. Influence of powder particle size distribution on complex viscosity and other properties of acrylic bone cement for vertebroplasty and kyphoplasty. J Biomed Mater Res B Appl Biomater 2006; 77: 98-103.

47. Hernandez L, Fernandez M, Collia F, Gurruchaga M, Goni I. Preparation of acrylic bone cements for vertebroplasty with bismuth salicylate as radiopaque agent. Biomaterials 2006; 27: 100-107.

48. Hernandez L, Vazquez B, Lopez-Bravo A, Parra J, Goni I, Gurruchaga M. Acrylic bone cements with bismuth salicylate: Behavior in simulated physiological conditions. J Biomed Mater Res A 2006.

49. Hernandez L, Goni I, Gurruchaga M. Incorporation of strontium hydroxyapatite to injectable acrylic bone cements. In: Transactions of the 20th European Conference on Biomaterials; 2006; Nantes, France. 37.

50. Gheduzzi S, Webb JJ, Miles AW. Mechanical characterisation of three percutaneous vertebroplasty biomaterials. J Mater Sci Mater Med 2006; 17: 421426.

51. Soltesz U, Schafer R, Jaeger R, Gopp U, Kuhn K-D. Fatigue testing of bone cements - comparison of testing arrangements. J of ASTM Inter 2005; 2: 1-11.

52. Wilke HJ, Neef $P$, Caimi M, Hoogland T, Claes LE. New in vivo measurements of pressures in the intervertebral disc in daily life. Spine 1999; 24: 755-762.

53. Cauich-Rodriguez JV, Vazquez-Torres $H$, Martinez-Richa A. Properties of THFMAPEMA and BMA-PEMA-based bone cements characterized by thermal analysis, FTIR and NMR. J Appl Biomater Biomech 2003; 1: 108-116.

54. Vallo CI, Schroeder WF. Properties of acrylic bone cements formulated with BisGMA. J Biomed Mater Res B Appl Biomater 2005; 74: 676-685.

55. De Santis R, Mollica F, Ambrosio L, Nicolais L, Ronca D. Dynamic mechanical behavior of PMMA based bone cements in wet environment. J Mater Sci Mater Med 2003; 14: 583-594.

56. La Rosa G, Risitano A. Thermographic methodology for rapid determination of the fatigue limit of materials and mechanical components. Int J Fatigue 2000; 22: 6573. 
$\mathbf{1 1 8}$ | Chapter 5 


\section{General Discussion}


Two discoveries that more or less revolutionized the world of medicine were:

1) The discovery of X-rays by Wilhelm Röntgen in 1895, which enabled surgeons to look into a human body without cutting it open. With the rise of the computer it became even possible to make 3D reconstructions using X-rays: a technique called computed tomography.

2) Since Harold Ridley in 1949 recognized that pilots in the airforce, who had suffered penetrating injuries to the eye from the plastic windshields of the aircraft, showed no adverse reactions to the material in the eye after the initial trauma had quieted, polymers were introduced into medicine [1]. Because of the versatility of polymers, they have found widespread use in all kinds of medical applications.

Unfortunately it is difficult to combine these discoveries, since polymers consist primarily of the lower elements $\mathrm{C}, \mathrm{H}, \mathrm{O}$ and $\mathrm{N}$, which makes it impossible to visualize them, using $\mathrm{X}$-rays, inside the human body. To date, this is overcome by the addition of metal markers or inorganic contrast agents to the polymeric biomaterial. These additions however, solely for the purpose of radiopacity, can have a negative effect on implant behaviour. Therefore we believe it is better to provide polymers with intrinsic radiopacity. This is achieved by covalently binding one (or more) iodine atom(s) to a polymer building block.

In this thesis the focus was on intrinsically radiopaque polymeric biomaterials for spinal surgery, more specifically a hydrogel prosthesis for nucleus pulposus replacement and an acrylic bone cement for the augmentation of vertebral compression fractures.

\section{Nucleus pulposus replacement}

When developing suitable replacements for parts of the human body, it becomes clear how hard it is to approach Nature's design. Million years of evolution have produced complex and perfectly working organs, in complete synergy. This is also true for the nucleus pulposus. It interacts synergistically with the annulus fibrosus and the vertebral end-plates. Hence, if the nucleus needs to be replaced, while the other structures are still functional, it would be wise to retain them.

The question often posed is why we didn't develop an injectable nucleus prosthesis. It would be even more minimally invasive. The main reason is that an injectable synthetic nucleus can also extrude through an annulus defect [2]. To counter that, the injected nucleus would have to solidify in situ (e.g. by 
polymerization of thermoreversible gelation) to make it more solid (but not too stiff). In our situation, this would mean that the injected mixture would have to contain monomers, which are very toxic substances in general. Hence it is not surprising that the most successful nucleus replacement to date, the PDN [3], is no injectable nucleus, but a pre-cured hydrogel.

Building on pioneering work of the PDN, we developed our own nucleus prosthesis from scratch. In our view, the PDN has two main drawbacks, which we wanted to address, being insufficient radiopacity (only markers) and an unnatural implant shape.

At this point we feel we have adequately addressed both drawbacks. The work so far culminated in the material T94_2, which fulfils the demands as stipulated in the Introduction to this thesis. In contrast to the PDN, this material is intrinsically radiopaque at a level that is sufficient for use in the vertebral column, and it can be lathe cut to the dimensions of the nucleus cavity (incorporating its swelling ability). When used clinically, either the material can be prepared in a range of sizes, and the surgeon can decide on the spot which size to implant, or the size of the prosthesis can be custom-made, based on $\mathrm{T}_{2^{-}}$ weighted MRI of the nucleus cavity of the patient in question [4].

Still, in the development of this nucleus replacement material, some compromises had to be made: Ideally, (i) the water content of the implant would be even higher, approximately $80 \%$ (like the natural nucleus). This can not be realized without doing concessions to the mechanical properties [5] and radiopacity; (ii) a highly hydrophilic (methacrylic) monomer would be available with a reactivity ratio comparable to 4IEMA, resulting in perfectly random copolymers, so no dissolution and consequent mass loss would occur in aqueous media. We could find no such monomer, so we had to resort to an appropriate chemical crosslinker; and (iii) the hydrogel is loaded with drugs, to stimulate the natural repair of the annulus. This can be the subject of future investigation.

Nonetheless, as stated above, we feel we have designed a viable nucleus prosthesis, featuring intrinsic radiopacity. Unfortunately, the road to actual clinical use of our nucleus prosthesis is still quite long. For one, fatigue tests need to be performed, preferably in a confined model, for a large number of cycles (85 million cycles, equal 40 years of use [6]). All the more since nucleus replacement is only useful in patients with a healthy (not too degenerated) annulus fibrosus, which are mainly young patients, meaning the implant has to function for a long time. To mimic the physiological situation, the loading protocol should be performed with a diurnal loading mode; for example 20 hours of cyclic compression followed by 4 hours of rest [7]. Also, animal 
experiments should demonstrate the biofunctionality and -compatibility of the prosthesis in situ. Interestingly, this prosthesis could also be used to treat back pain in dogs. Together with experts in veterinary medicine we are exploring this potential application. At the time of this writing, animal experiments in a rabbit model are prepared and ready to start. All the necessary approvals are obtained and nucleus implants are machined and ethylene oxide sterilized. Several orthopaedic surgeons are willing to perform the implantations. Hopefully these experiments can start in the near future. After that, the final step before clinical use of our radiopaque hydrogel, is a clinical pilot study; the new prosthesis is implanted in selected patients, with written consent, who are monitored for a certain amount of time. Fortunately, already one patient is available... the amateur triathlete who emailed me (Appendix 1).

\section{Acrylic bone cement}

The cement we developed for use in vertebroplasty or balloon kyphoplasty, in contrast to the nucleus prosthesis, was not developed from scratch. Earlier we prepared an all-polymeric intrinsically radiopaque bone cement for use in cemented total joint replacements [8]. Only for the present application the radiopacity had to be increased.

The use of acrylic bone cement, also in contrast to the nucleus prosthesis, does involve the injection of toxic monomers into the human body. Although we might feel this is a crude method, it is an established technique with a high success rate. The question that remains is how it was ever approved (by the FDA) in the first place.

Realizing there are no official standards for vertebroplasty cements, the aim of the study was to design a cement that performs equally to commercially available $\mathrm{BaSO}_{4}$-containing cement, but that lacks the inorganic radiopacifier with its inherent deleterious effects.

The iodine-containing cement is still in the development phase and some issues need to be addressed. The field in which we feel much profit can still be gained is in the rheological behaviour of the cement after mixing, especially in view of the fact that for this application, the cement needs to be injected through a needle. The viscosity is greatly influenced by the liquid monomer-to-powder ratio [9], the size (distribution) of the microspheres in the powder [10] and by the presence of barium sulphate (personal observation). The particle size distribution of our own microspheres is quite broad and we are currently working to make it more uniform. We noticed that the presence of barium 
sulphate causes granularity of the cement mixture, another reason to exclude it from bone cements.

The greatest challenge we are faced with at this point, is to convince the conservative medical world to use our all-polymeric cement instead of cements with added radiopaque agents, and to convince an investor or company to commercialize it.

\section{References}

1. Boelen EJH. Novel iodine containing biomaterials for use in intraocular lenses. Eindhoven: Technical University Eindhoven; 2002. Master thesis.

2. Goins ML, Wimberley DW, Yuan PS, Fitzhenry LN, Vaccaro AR. Nucleus pulposus replacement: an emerging technology. Spine J 2005; 5: 317S-324S.

3. Ray CD. The PDN prosthetic disc-nucleus device. Eur Spine J 2002; 11 Suppl 2: S137-142.

4. Ghosh P (ed.). The biology of the intervertebral disc. vol. 2. CRC Press; 1988.

5. Anseth KS, Bowman CN, Brannon-Peppas L. Mechanical properties of hydrogels and their experimental determination. Biomaterials 1996; 17: 1647-1657.

6. Kostuik JP. Intervertebral disc replacement. Experimental study. Clin Orthop Relat Res 1997: 27-41.

7. Bertagnoli R, Sabatino CT, Edwards JT, Gontarz GA, Prewett A, Parsons JR. Mechanical testing of a novel hydrogel nucleus replacement implant. Spine J 2005; 5: 672-681.

8. van Hooy-Corstjens CSJ, Govaert LE, Spoelstra AB, Bulstra SK, Wetzels GM, Koole $\mathrm{LH}$. Mechanical behaviour of a new acrylic radiopaque iodine-containing bone cement. Biomaterials 2004; 25: 2657-2667.

9. Jasper LE, Deramond H, Mathis JM, Belkoff SM. The effect of monomer-topowder ratio on the material properties of cranioplastic. Bone 1999; 25: 27S-29S.

10. Hernandez L, Gurruchaga M, Goni I. Influence of powder particle size distribution on complex viscosity and other properties of acrylic bone cement for vertebroplasty and kyphoplasty. J Biomed Mater Res B Appl Biomater 2006; 77: 98-103. 
124 | Chapter 6 


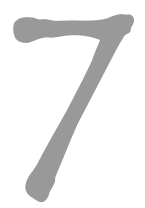

Appendix 


\section{E-mail from an amateur triathlete with back pain}

Dear Erik

I have today read with much interest your article as mentioned above as I have suffered degenerative disc disease between Lumbar 5 and Sacrum 1. Currently I am awaiting an MRI Scan (within approximately the next 4 months). The degenerated disc has been detected by full neck and spine X-rays.

I am a teacher of Chemistry at a Secondary School (children 12-18 years) in Perth Scotland, and until two years ago was extremely fit and active - involved in long distance triathlon races; swim $2.4 \mathrm{~km}$, cycle $180 \mathrm{~km}$ and run a marathon of $42 \mathrm{~km}$ as a continuous race. No doubt this has contributed to the degeneration. I am also studying part-time for an MSc in Biological Nuclear Magnetic Resonance Spectroscopy, so would also have an interest in the imaging of these gels by MRI scans.

I am able to understand the chemistry behind what you are doing and desperate to regain my ability to swim, cycle and run. I competed as an amateur to a high level in National Championships. I am only 36 years old.

I appreciate that further testing and trials of the hydrogels are needed before they become available in routine surgery. Can you give me an indication as to when these gels may become available as a cure???

I am willing to offer myself as a patient for use in clinical trials if this would assist your research. My mother and father-in-law are both Dutch and therefore I would have no difficulty in coming over to The Netherlands if needed.

I look forward to hearing from you.

Regards...........JIM SCANLAN 


\section{$2{ }^{1} \mathrm{H}-\mathrm{NMR}$ analysis of the copolymerization of NVP and 4IEMA}

For the determination of the reactivity ratios, we focused on three specific resonances, i.e. (a) aromatic protons of 4IEMA (either unreacted 4IEMA ( $\delta 7.9$ ppm and $\delta 7.7 \mathrm{ppm}$ ), or built-in 4IEMA (broadened signals at $\delta 7.8 \mathrm{ppm}$ and $\delta$ $7.7 \mathrm{ppm})$ ), (b) the vinyl proton of NVP (four line pattern at $\delta 7.0 \mathrm{ppm}$ ), (c) the vinyl protons of 4IEMA (singlets at $\delta 6.0 \mathrm{ppm}$ and $\delta 5.6 \mathrm{ppm}$ ). Figure 2 (Chapter 2) shows these resonances in detail, for $f_{\text {4IEMA }}=0.43$, at $0 \%$ conversion (Figure 2A) and at a conversion of 15\% (Figure 2B). Integration of the patterns $a, b$ and $c$ provided detailed information about the conversion and about the composition of the copolymer formed at the time of the acquisition. The ${ }^{1} \mathrm{H}$-NMR spectra were analyzed in two ways: determination of the ratio consumed NVP : consumed 4IEMA at conversion in the range (i) $4-6 \%$ and (ii) $13-16 \%$. At low conversion (i), the composition drift can be neglected. At high conversion (ii), it is possible to measure the consumption of NVP with higher accuracy. It was uncertain a priori which of the two approaches would yield more reliable results in terms of reactivity ratios $r_{4 I E M A}$ and $r_{N V P}$ for this particular reaction.

To make a proper comparison between the different spectra in time, it was assumed that the combined integral of the four aromatic hydrogen signals remains constant during the reaction (int. $a=$ int. $\left(a_{1}+a_{2}+a_{1}{ }^{\prime}+a_{2}{ }^{\prime}\right)=4$ ), while the integrals of signals $b$ and $c$ decrease as NVP and 4IEMA are built in the growing copolymer chains. From this decrease, the incorporation of monomer in the polymer chain, i.e. the copolymer composition, is deduced. At time $t$, the conversion was calculated using equation 1 :

conversion (\%) $=100 \times \frac{(\operatorname{int} . b+\operatorname{int} . \bar{c})_{t=0}-(\operatorname{int} . b+\operatorname{int} . \bar{c})_{t}}{(\operatorname{int} . b+\operatorname{int} . \bar{c})_{t=0}}$

In which $\bar{c}$ is the average of $c_{1}$ and $c_{2}$.

At this conversion, the fraction of built-in 4IEMA is obtained using equation 2:

$$
F_{4 I E M A}=\frac{\operatorname{int} . \bar{C}}{(\operatorname{int} . b+\operatorname{int} . \bar{c})_{t=0}-(\operatorname{int} . b+\operatorname{int} . \bar{c})_{t}}
$$


From the copolymer composition and the monomer feed composition, the reactivity ratios can be calculated according to equation $3[1,2]$.

$$
\frac{f_{4 I E M A}\left(1-2 F_{4 I E M A}\right)}{F_{4 I E M A}\left(1-f_{4 I E M A}\right)}=r_{\text {NVP }}+\left(\frac{f_{4 I E M A}^{2}\left(F_{4 I E M A}-1\right)}{F_{4 I E M A}\left(1-f_{4 I E M A}\right)^{2}}\right) r_{4 I E M A}
$$

Where $F_{4 I E M A}$ is the molar fraction of 4IEMA in the copolymer and $f_{\text {NVP }}$ and $f_{4 I E M A}$ are the molar fractions of NVP and 4IEMA, respectively, in the feed. $r_{4 \text { IEMA }}$ and $r_{\mathrm{NVP}}$ are the reactivity ratios.

When plotting the left-hand side of the equation against the coefficient of $r_{4 \text { IEMA, }}$ $r_{4 \text { IEMA }}$ and $r_{\text {NVP }}$ can be determined, by drawing a linear trendline through the data points.

\section{References}

1. Odian G. Principles of polymerization. Wiley-Interscience; 1991.

2. Young RJ, Lovell PA. Introduction to polymers. London: Chapman \& Hall; 1991. 


\section{Title page Patent}

(12) INTERNATIONAL APPLICATION PUBLISHED UNDER THE PATENT COOPERATION TREATY (PCT)

(19) World Intellectual Property Organization International Burcau

(43) International Publication Date 16 March 2006 (16.03.2006)

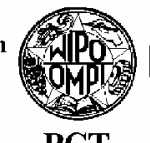

PCT
|||||||||||||||||||||||||||||||||||||||||||||||||||||||||||||||||||||||||||||||||||||||

(10) International Publication Number
(51) International Patent Classification: A6IIL $27 / 52(2006.01) \quad$ A6/L.27/50 (2006.01)

(21) International Application Number: PCW/NL2005/000650

(22) International Filing Date:

8 September 2005 (08.09.2005)

(25) Fìling Language:

Dutch

(26) Publication I anguage:

Tinglish

(30) Priority Data:

$04077514.0 \quad 10$ September $2004(10.09 .2004) \quad$ EP

(71) Applicant (for all designated States except $1 / 5)$ : STICHTING DUTCH POLYMER INSTITUTE [NL/NL]; jOHN f. kENNEDYLAAN 2, NL-5612 $\Lambda$ B Eindhoven (NL).

(72) Inventors; and

(75) Inventors/Applicunts (for US only): KOOLE, Levinus Hendrik [NL/NL]; Bolderik 9, NL-6271-EC Gulpen (NL). BOELEN, Erik, Johannes, Herman $|\mathrm{NL} / \mathrm{NL}|$; $4 \mathrm{n}$ byerstraat Noord 147, NI,-6225 IDD Maastricht (NI.). VAN HOOY-CORSTJENS, Catharina, Sibilla, Josephine [NL/NL]: Burgemeester Schoolmeestersstraat 3, NL-6116 $\mathrm{BX}$ Roosteren (NL).
(74) Agents: NIEUWKAMP, Johannes, Gerardus, Maria et al.; DSM Intelleclual Property, PO. Box 9, NI.-6160 MA Geleen (NL).

(81) Designated States (uniess otherwise indicated, for every kind of national protection available): $\mathrm{AE}, \mathrm{AG}, \mathrm{AL}, \mathrm{AM}$ $\Lambda \mathrm{T}, \Lambda \mathrm{U}, \Lambda Z, \mathrm{BA}, \mathrm{BB}, \mathrm{BG}, \mathrm{BR}, \mathrm{BW}, \mathrm{BY}, \mathrm{BZ}, \mathrm{C} \Lambda, \mathrm{CI}, \mathrm{CN}$, CO, CR, CU, CL, DE, DK, DM, DZ, EC, EE, EG, ES, Fl, GB, GD, GT, GH, GM, HR, HU, ID, II, IN, IS, JP, KT, KG, KM, KP, KR, KZ, LC, LK, LR, LS, LT, LU, LV, MA, MD, MG, MK, MN, MW, MX, MZ, NA, NG, NI, NO, NZ OM, PG, PH, I'L, I'I, RO, RU, SC, SD, SE, SG, SK, SL, SM, SY, TJ, TM, TN, TR, TT, TZ, UJA, UG, US, UZ, VC VN, YU, ZA, ZM, ZW.

(84) Designated States (unless oitherwise indicated, for every kind of regional protecion avalable): ARIPO (BW, GII, GM, KE, LS, MW, MZ, NA, SD, SL, SZ, TZ, UG, ZM, ZW), Eurasian (AM, AL, BY, KG, KZ, MD, RU, IJ, 'TM), Turopean (AT, BT, BG, CH, CY, C7, DT, DK, TE, ГS, ПT, FR, GB, GR, IIU, IE, IS, IT, LT, LU, LV, MC, NL, PL, PT, RO, SE, SI, SK, IR), OAPI (BF, BJ, CF, CG, Cl, CM, GA, GN, GQ, GW, ML, MR, NE, SN, TD, 'TG)

Published:

- with international search report

[Continued on next page]

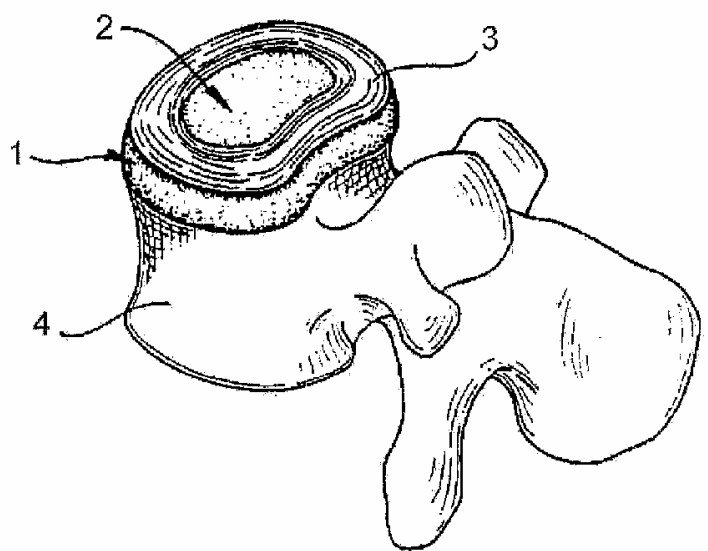

(57) Abstract: 'This invention relates to a hydrogel based on a copolymer comprising (a) at least one monomer comprising covalently bound iodine or bromine, and; (b) al least one hydrophilic monomer, wherein the hydrogel, when fully hydraled, has an elastic modulus between $0.01-100 \mathrm{MPa}$ at $37^{\circ} \mathrm{C}$. The invention also relates to a prosthetic nucleus comprising the hydrogel according to the invention and a method for making the prosthesis. 


\section{Comment on the article described in Chapter 3}

\section{Beating back pain}

\section{January 2006}

Erik Boelen and colleagues at Maastricht University, the Netherlands, have developed a hydrogel biomaterial that, unlike existing implants, completely fills the cavity left by a removed disc nucleus. These spinal implants can also mimic natural discs more closely than existing implants.

The key to the new hydrogel is its swelling property. The material is implanted through a small opening as a dry xerogel. Once in situ, the gel takes up water, expanding to fill the cavity. Additionally, the gel has been designed to be clearly visible by magnetic resonance imaging (MRI) and computer tomography (CT), which not only allows doctors to accurately place the implant during surgery, but can later be used to confirm that the gel has swollen sufficiently.

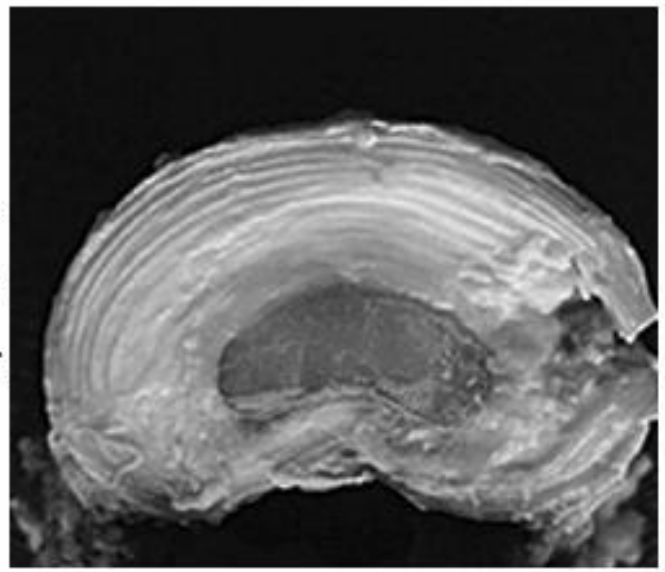

Traditionally, surgeons treating degenerative disc disease have had to resort to spinal fusion, removing the diseased disc and locking together the two adjacent vertebrae. However, the resulting loss of local mobility places more strain on neighbouring discs, potentially triggering more back problems in the future.

"The next step for Boelen is to study the long term fatigue behaviour of his biomaterial"
If the disease is caught at an early stage the diseased disc can be replaced by Boelen's artificial, hydrogel-based disc, which can be inserted into the spine. Not only is this

procedure is far less invasive than spinal fusion, but full spinal mobility is retained.

Initial testing in animal models has shown that the gel does not cause significant side effects. The next step for Boelen is to study the long term fatigue behaviour of his biomaterial, which will have to cope with significant loads during bending and lifting, and to ensure that the implant will not leak out of the cavity.

James R Mitchell Crow

References

E J H Boelen, C S J van Hooy-Corstjens, M J J Gijbels, S K Bulstra, A van Ooij, L W van Rhijn and L H Koole, J. Mater. Chem, 2005 (Dol: 10.1039/b515904k) 
Summary / Samenvatting

List of Publications

Curriculum Vitae

Dankwoord 


\section{Summary}

Back pain is a major problem in society, and the costs related to it are enormous. Disc problems and compression fractures are among the most common ailments to the back. With our present knowledge on radiopaque polymers, we hoped to contribute to the field of spinal surgery, by providing orthopaedic surgeons with intrinsically radiopaque biomaterials. Intrinsic radiopacity is essential for completely visualizing an implanted biomaterial. The basis for rendering our biomaterials radiopaque lies with the monomer 4IEMA, which contains covalently bound iodine. This monomer can be copolymerized with other monomers, depending on the desired application.

Chapter $\mathbf{2}$ deals with a first investigation towards radiopaque hydrogels for nucleus pulposus replacement. Ideally a nucleus replacement mimics the functions and behaviour of the natural nucleus. In an attempt to create radiopaque hydrogels, the iodine-containing monomer 4IEMA was copolymerized with either one of the hydrophilic monomers NVP and HEMA in different ratios. The copolymerization reaction of NVP + 4IEMA was studied in detail using ${ }^{1} \mathrm{H}-\mathrm{NMR}$. It was found that 4IEMA is far more reactive than NVP. For the nucleus prosthesis eight different copolymers were synthesized with 5 $20 \mathrm{~mol} \%$ 4IEMA. These copolymers could be swollen in aqueous media to yield hydrogels. The resulting hydrogels were investigated for their water content, cytotoxicity, X-ray opacity, stiffness, rheological properties and hysteresis.

The reactivity ratios of NVP and 4IEMA clarified the polymerization dynamics and explained the mass loss of the NVP-based hydrogels in water. This mass loss is due to the dissolution of NVP oligomers. It was also found that the incorporation of 4IEMA has a significant effect on the water content of the hydrogels, but not on the cytotoxicity; all hydrogels were non-cytotoxic. The Xray opacity of all swollen copolymers was sufficient for visualizing them in between two vertebrae. The hydrogels with the highest water content came closest to the natural nucleus pulposus in terms of rheological behaviour. The hydrogels containing 5 mol\% 4IEMA were in range of the desired stiffness, the others were too stiff for the intended application. All hydrogels displayed hysteresis when subjected to cyclic loading, as does the natural nucleus. From this study we concluded that approximately 5 mol\% 4IEMA in combination with either NVP or HEMA results in promising biocompatible, radiopaque hydrogels for replacing the nucleus pulposus. 
Consequently, in Chapter $\mathbf{3}$ we continued the investigation of the radiopaque hydrogels. This time the focus was on biocompatibility in vivo, implantability and visualization using X-rays. Copolymers were prepared with 6 mol\% 4IEMA and 94 mol\% of either NVP or HEMA, indicated by N94 and H94 respectively. The stiffness and swelling of both materials was studied and small discs were implanted in mice. A prototype nucleus prosthesis was lathe cut from N94 and implanted in a lumbar disc of a porcine cadaver. The porcine spine was then visualized using CT and MRI. This study demonstrated the feasibility of our approach, the biocompatibility of the materials and the excellent visibility of the prototype prosthesis in situ using either CT or MRI.

Realizing that both N94 and H94 still have their shortcomings, being oligomer dissolution and low swelling capacity respectively, we fine-tuned these materials as described in Chapter 4. To overcome the dissolution of NVP-oligomers from N94, a chemical crosslinker was introduced. Also, NVP, HEMA and 4IEMA were combined in a new terpolymer, in an attempt to combine the high water content of NVP-based hydrogels with the elasticity of the HEMA-based materials. This new terpolymer was also chemically crosslinked. The new crosslinked materials were evaluated and compared to $\mathrm{N} 94$ and $\mathrm{H} 94$ for an array of relevant properties. The mass loss was significantly reduced for the crosslinked materials and they also seemed to perform better mechanically. Only the water content was somewhat lower than N94. A preliminary fatigue experiment on the new materials suggested fatigue resistance.

Especially the terpolymer has great potential to serve as a functional nucleus prosthesis and it will be evaluated in vivo in a suitable animal model in the near future.

In Chapter 5 a switch has been made from the radiopaque hydrogel to a highly radiopaque cement for the treatment of osteoporotic vertebral compression fractures. This chapter describes the comparison of 2 highly radiopaque cements for use in vertebroplasty or kyphoplasty. One cement was made radiopaque by the incorporation of iodine-containing copolymer in the powder phase of the cement (IO cement). In the other cement, radiopacity was introduced by the addition of barium sulphate (BA cement), as is commonly done in clinical practice. Because the addition of high levels of barium sulphate has raised concerns in the orthopaedic community, the all-polymeric IO cement was developed and compared to BA cement. Both cements were tested for a wide array of relevant properties. For a majority of these properties, the difference between these cements was not significant. Therefore we believe that IO cement is a viable alternative to BA cement for use in vertebroplasty and kyphoplasty. 


\section{Samenvatting}

Rugpijn is een groot probleem in de samenleving, en de kosten die ermee gepaard gaan, zijn enorm. Problemen met een tussenwervelschijf en compressiefracturen behoren tot de meest voorkomende rugaandoeningen. Met onze huidige kennis van röntgenzichtbare polymeren hoopten we te kunnen bijdragen aan de wervelkolomchirurgie, door orthopedisch chirurgen te voorzien van intrinsiek röntgenzichtbare biomaterialen. Intrinsieke röntgenzichtbaarheid is essentieel voor het compleet in beeld brengen van een geïmplanteerd biomateriaal. De basis voor het röntgenzichtbaar maken van onze biomaterialen ligt bij het monomeer 4IEMA, dat covalent gebonden jood bevat. Dit monomeer kan copolymeriseren met andere monomeren, afhankelijk van de gewenste toepassing.

Hoofdstuk 2 behandelt een eerste studie naar röntgenzichtbare hydrogelen om de nucleus pulposus te vervangen. Idealiter bootst een vervanging van de nucleus, de functies en het gedrag van een natuurlijke nucleus na. In een poging röntgenzichtbare hydrogelen te maken, is het jood-houdende monomeer 4IEMA gecopolymeriseerd met een van de hydrofiele monomeren NVP en HEMA, in verschillende ratio's. De copolymerisatie-reactie van NVP + 4IEMA is in detail bestudeerd met ${ }^{1} \mathrm{H}-\mathrm{NMR}$. Hieruit bleek dat 4IEMA veel reactiever is dan NVP. Voor de nucleus prothese zijn acht verschillende copolymeren gemaakt met 5 - 20 mol\% 4IEMA. Deze copolymeren konden zwellen in waterige oplossingen tot hydrogelen. Deze hydrogelen zijn onderzocht op hun waterpercentage, cytotoxiciteit, röntgenzichtbaarheid, stijfheid, reologische eigenschappen en hysterese.

De reactiviteitsratio's van NVP en 4IEMA helderden de polymerisatie-dynamica op en verklaarden het massaverlies van de NVP-hydrogelen in water. Dit massaverlies is het gevolg van het oplossen van NVP-oligomeren. Tevens bleek dat het toevoegen van 4IEMA een significant effect had op het waterpercentage van de hydrogelen, maar niet op de cytotoxiciteit; geen van de hydrogelen was toxisch. De röntgenzichtbaarheid van alle gezwollen copolymeren was voldoende om ze in beeld te brengen tussen twee wervels. De hydrogelen met het hoogste waterpercentage leken reologisch het meest op de natuurlijke nucleus pulposus. De hydrogelen met 5 mol\% 4IEMA zaten in het gebied van de gewenste stijfheid, de overigen waren te stijf voor de bedoelde toepassing. Alle hydrogelen vertoonden hysterese, net als de natuurlijke nucleus. Uit deze studie concludeerden wij dat ongeveer 5 mol\% 4IEMA, samen met NVP of 
HEMA, resulteert in veelbelovende, biocompatibele, röntgenzichtbare hydrogelen voor het vervangen van de nucleus pulposus.

Vervolgens zijn we in Hoofdstuk 3 verder gegaan met het bestuderen van de röntgenzichtbare hydrogelen. Dit keer lag de focus op biocompatibiliteit in vivo, implanteerbaarheid en röntgenzichtbaarheid. Hiervoor werden copolymeren gemaakt met 6 mol\% 4IEMA en 94 mol\% NVP of HEMA, aangeduid respectievelijk als N94 en H94. De stijfheid en het zwellen van beide materialen is bestudeerd en kleine schijfjes zijn geïmplanteerd in muizen. Een prototype nucleus prothese van N94 werd gemaakt op de draaibank en geïmplanteerd in een lumbale tussenwervelschijf van een varkenskadaver. De wervelkolom werd vervolgens in beeld gebracht met CT en MRI. Deze studie toonde de uitvoerbaarheid van onze benadering, de biocompatibiliteit van de materialen en de uitstekende zichtbaarheid van ons prototype in situ met zowel CT als MRI.

Omdat we ons realiseerden dat zowel N94 als H94 nog steeds tekortkomingen hebben, zijnde respectievelijk massaverlies en lage zwelcapaciteit, hebben we deze materialen verder geoptimaliseerd, zoals beschreven in Hoofdstuk 4. Om het oplossen van oligomeren uit N94 te voorkomen, werd een chemische crosslinker geïntroduceerd. Tevens zijn NVP, HEMA en 4IEMA gecombineerd in een nieuw terpolymeer, in een poging het hoge waterpercentage van de NVPhydrogelen te combineren met de elasticiteit van de HEMA-hydrogelen. Dit nieuwe terpolymeer is ook gecrosslinkt. De nieuwe gecrosslinkte materialen zijn getest op een aantal relevante eigenschappen en vergeleken met N94 en H94. Het massaverlies was aanzienlijk verminderd bij de gecrosslinkte materialen en ze leken mechanisch ook beter te voldoen. Enkel het waterpercentage was iets lager dan N94. Een voorlopig vermoeiingsexperiment leek te duiden op vermoeiingsweerstand.

Voornamelijk het terpolymeer is veelbelovend als functionele nucleus prothese, en dit zal in de nabije toekomst in vivo worden getest in een geschikt diermodel.

In Hoofdstuk 5 maken we een omschakeling naar een extra röntgenzichtbaar cement ter behandeling van osteoporotische compressiefracturen in de wervels. Dit hoofdstuk beschrijft de vergelijking van twee extra röntgenzichtbare cementen voor gebruik in vertebroplastie of kyphoplastie. Een cement is röntgenzichtbaar gemaakt door toevoeging van een jood-houdend copolymeer in het poeder van het cement (IO cement), het andere is röntgenzichtbaar gemaakt door bariumsulfaat toe te voegen (BA cement), zoals gewoonlijk gedaan wordt in de kliniek. Omdat in de orthopedische wereld ongerustheid bestaat over de toevoeging van grote hoeveelheden bariumsulfaat, werd het 
volledig-polymere IO cement ontwikkeld en vergeleken met BA cement. Beide cementen zijn getest op een aantal relevante eigenschappen. Voor de meeste hiervan was het verschil tussen de cementen niet significant. Daarom denken wij dat IO cement een goed alternatief is voor BA cement in vertebroplastie en kyphoplastie. 


\section{List of Publications}

\section{Papers}

- Camiel C. Peerlings, Hans H. Hanssen, Raymond T. Bevers, Erik J.H. Boelen, Bram J. Stelt, Eva J. Korthagen, Leo $\mathrm{H}$. Koole. Heparin release from slipperywhen-wet guide wires for intravascular use. J Biomed Mater Res 63 (2002) 692-698.

- Erik J.H. Boelen, Catharina S.J. van Hooy-Corstjens, Sjoerd K. Bulstra, André van Ooij, Lodewijk W. van Rhijn, Leo $\mathrm{H}$. Koole. Intrinsically Radiopaque Hydrogels for Nucleus Pulposus Replacement. Biomaterials 26 (2005) 66746683.

- Erik J.H. Boelen, Catharina S.J. van Hooy-Corstjens, Marion J.J. Gijbels, Sjoerd K. Bulstra, André van Ooij, Lodewijk W. van Rhijn, Leo H. Koole. Preliminary Evaluation of New Intrinsically Radiopaque Hydrogels for Replacing the Nucleus Pulposus. J Mater Chem 16 (2006) 824-828.

- Erik J.H. Boelen, Leo H. Koole, Lodewijk W. van Rhijn, Catharina S.J. van Hooy-Corstjens. Towards a functional radiopaque hydrogel for nucleus pulposus replacement. J Biomed Mater Res B Appl Biomater in press.

- Erik J.H. Boelen, Gladius Lewis, Jie Xu, Tristan Slots, Leo H. Koole, Catharina S.J. van Hooy-Corstjens. Evaluation of a Higly Radiopaque Iodine-Containing Acrylic Bone Cement for Use in Augmentation of Vertebral Compression Fractures. J Biomed Mater Res A submitted.

\section{Patent}

- Leo H. Koole, Erik J.H. Boelen, Catharina S.J. van Hooy-Corstjens. Radiopaque Prosthetic Intervertebral Disc Nucleus. WO2006/028370.

\section{Abstracts}

- Erik J.H. Boelen, Catharina S.J. van Hooy-Corstjens, Sjoerd K. Bulstra, André van Ooij, Lodewijk W. van Rhijn, Leo H. Koole. Annual meeting of the Dutch Society for Biomaterials and Tissue Engineering (NBTE) 2003, 2004, 2005. 
- Erik J.H. Boelen, Catharina S.J. van Hooy-Corstjens, Sjoerd K. Bulstra, André van Ooij, Lodewijk W. van Rhijn, Leo $\mathrm{H}$. Koole. Integrated Biomedical Engineering for Restoration of Human Function (iBME) Conference. 2003, 2004, 2005.

- Erik J.H. Boelen, Raymond T.J. Bevers, Leo H. Koole. New Transparent Copolymers with a High Refractive Index. Transactions of the 7th World Biomaterials Congress, Sydney, Australia (2004) 113.

- Erik J.H. Boelen, Catharina S.J. van Hooy-Corstjens, Sjoerd K. Bulstra, André van Ooij, Lodewijk W. van Rhijn, Leo H. Koole. Radiopaque Hydrogels for Possible Nucleus Pulposus Replacement. Transactions of the 7th World Biomaterials Congress, Sydney, Australia (2004) 1557.

- Erik J.H. Boelen, Catharina S.J. van Hooy-Corstjens, André van Ooij, Lodewijk W. van Rhijn, Leo H. Koole. Preclinical Evaluation of Intrinsically Radiopaque Hydrogels for Replacing the Nucleus Pulposus. ECM VI - Spinal Motion Segment, Davos, Switzerland. Abstract also published in Eur Cell Mater 10 S3 (2005) 10.

- Erik J.H. Boelen, Leo H. Koole, Lodewijk W. van Rhijn, Catharina S.J. van Hooy-Corstjens. Intrinsically Radiopaque Hydrogels for Replacing the Nucleus Pulposus. Transactions of the 8th New Jersey Symposium on Biomaterials Science, New Brunswick, NJ, USA (2006) 58.

\section{Awards}

- Oral Presentation Award, Nederlandse Vereniging voor Biomaterialen en Tissue Engineering (NBTE), Lunteren, NL 2005.

- Student Travel Award, The $8^{\text {th }}$ New Jersey Symposium on Biomaterials Science, New Brunswick, NJ, USA 2006. 


\section{Curriculum Vitae}

Erik Johannes Herman Boelen was born on April 13, 1979, in Meers, The Netherlands. After graduating from secondary school (VWO) SG Groenewald, Stein in 1997, he started the study Biomedical Engineering at the Eindhoven University of Technology. His interest in biomaterials was quickly obvious, and he came in contact with the biomaterials group of Prof. Dr. Ir. L.H. Koole at the University of Maastricht in his $4^{\text {th }}$ year for a multidisciplinary project on heparinreleasing guide-wires. He then decided to do a graduation project on high refractive index intraocular lenses in the same group. As a part of this project, he worked for 3 months at the Commonwealth Scientific and Industrial Research Organization (CSIRO) in Melbourne, Australia. Also during his graduation project, he obtained the status of article 9 functionary according to the Law on Animal Experiments. In December 2002 he obtained his Masters degree in Biomedical Engineering. In January 2003 he started the PhD-project on intrinsically radiopaque biomaterials under supervision of Prof. Dr. Ir. L.H. Koole, which led to this thesis.

Erik Johannes Herman Boelen werd geboren op 13 april 1979, te Meers. Na het behalen van zijn VWO diploma aan SG Groenewald in Stein in 1997, begon hij met de studie Biomedische Technologie aan de Technische Universiteit van Eindhoven. Zijn interesse voor biomaterialen was al snel duidelijk en hij kwam tijdens zijn $4^{e}$ jaar in aanraking met de biomaterialen groep van Prof. Dr. Ir. L.H. Koole, voor een multidisciplinair project over heparine afgifte van voerdraden. Hij besloot toen om zijn afstudeerproject in dezelfde groep te doen, aan intraoculaire lenzen met een hoge brekingsindex. In het kader van dit project heeft hij 3 maanden gewerkt bij CSIRO in Melbourne in Australië. Ook tijdens zijn afstudeerproject is hij Artikel 9 functionaris geworden volgens de Wet op de dierproeven. In december 2002 haalde hij zijn Ingenieurs diploma (ir.) in de Biomedische Technologie. In januari 2003 is hij het AIO-project begonnen over intrinsiek röntgenzichtbare biomaterialen, onder toezicht van Prof. Dr. Ir. L.H. Koole, dat geleid heeft tot dit proefschrift. 


\section{Dankwoord}

Het einde van dit proefschrift markeert ook het einde van mijn promotie. Graag wil ik van de gelegenheid gebruik maken terug te blikken op mijn AIO-tijd aan de Universiteit Maastricht en dan met name de mensen voor het voetlicht te brengen die gedurende die tijd de revue zijn gepasseerd. Vooral wil ik dan de mensen bedanken die een substantiële bijdrage hebben geleverd aan mijn onderzoek.

Allereerst natuurlijk Leo, mijn promotor. Leo, het is heel fijn dat ik altijd bij je kon binnenlopen. Tevens is het plezierig dat jij je zo direct met alle onderzoeken bemoeit en me toch de vrijheid hebt gelaten om bepaalde 'omstreden' experimenten uit te voeren. Nimmer verloor je het doel uit het oog en altijd wist je mijn wetenschappelijke output te versterken. Bedankt dat ik onder jouw bezielende leiding mocht promoveren.

Tosca, mijn copromotor, dagelijkse begeleidster en kamergenote. Jij was altijd mijn eerste aanspreekpunt en ik kon alles met je bespreken. Veel dank voor alle nuttige, al dan niet wetenschappelijke, discussies. Jouw kritiek was altijd goed onderbouwd en verhelderend. Bedankt voor je begeleiding en gezelligheid.

Sjoerd, mijn $2^{\mathrm{e}}$ promotor en de drijfveer achter het ontstaan van het project. Bedankt dat je zelfs vanuit Groningen mijn project hebt ondersteund.

Ons kleine groepje bij Biomaterialen; Menno, Yvette, Ketie en Rachel wil ik bedanken voor hun collegialiteit, interesse en hulp bij mijn onderzoek. Veel succes met jullie eigen onderzoeken.

Tevens een woord van dank aan:

Het Dutch Polymer Institute (DPI) voor de financiering van het project en de begeleiding bij het opstellen van het patent; De heren orthopeden, te weten André van Ooij, Lodewijk van Rhijn en aanstormend orthopeed Pieter Emans voor hun inbreng en enthousiasme; Joost, Frank en Lou van IDEE voor het maken van de 'sjiefkes' en de 'boontjes'; De mensen bij het CPV en Arne voor de hulp bij de dierproeven en röntgenopnamen; De heren van de SEM voor hun hulp bij de electronenmicroscoop; Geert Wijnhoven (CT) en Henk Schoenmakers (MRI) voor het maken van opnames tussen de patiënten door; Tom Engels van de TU in Eindhoven voor zijn assistentie bij de vermoeiingsexperimenten; Prof. Hazewinkel en dr. Meij (Universiteit Utrecht) voor hun enorme inzet om samen een grote dierproef uit te voeren. Hopelijk kan er in de nabij toekomst mee gestart worden; Tristan voor zijn uitstekende onderzoek naar het vertebroplastie-cement; Paul Willems voor de assistentie bij 
onze drukbank; Kirsten voor de 'staafmixer'; Marion Gijbels voor de interpretatie van de histologie; Leden van de beoordelingscommissie voor het lezen van dit proefschrift; Trees, die de hele gang draaiende houdt en mij wegwijs maakte in de bureaucratie; Also I would like to thank Gladius Lewis for the pleasant cooperation and his contribution to our research.

Er zijn natuurlijk ook een hoop mensen, verbonden aan de universiteit en daarbuiten, die mijn tijd (hier) onvergetelijk hebben gemaakt, maar die niet of nauwelijks betrokken waren bij mijn onderzoek. Aangezien ik ook wel weet dat velen als eerste (en sommigen als enige) het dankwoord lezen, wil ik deze mensen die mijn AIO-tijd hebben opgeleukt hier kort noemen, anders ga ik mijn boekje te buiten!

Iedereen van de BMT-groep, Kris en de männer van de zesde, Paola, Imke en de overige AIO's bij Biochemie, Niko, Damia, de orthopedie-groep op de $2^{\mathrm{e}}$ en de dames van de $2^{\mathrm{e}}(\mathrm{HB})$, de heren van Sittardia, Jeroen en nog meer leuke mensen die ik ontmoet heb op congressen en al mijn vriendjes en vriendinnetjes: Bedankt! Ook wil ik alle familieleden bedanken die mijn promotieonderzoek met veel interesse hebben gevolgd.

Een speciaal woordje wil ik nog richten tot Bram, mijn inmiddels ex-roomy. Je bent een echte vriend. Ik wens jou en Rosemarie het allerbeste voor de toekomst en veel sterkte in het buitenland.

Mijn pappie en mammie wil ik bedanken voor alle steun en vertrouwen. Jullie zijn er altijd voor mij. Merci pour tout!

Inge, mien Ingelke. Op jou kan ik altijd terugvallen. Ik ben ongelofelijk blij dat jij aan mijn zijde staat bij deze belangrijke stap in mijn leven. Dat we samen nog maar vele stappen, zelfs hele wandelingen, mogen ondernemen!

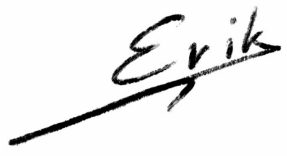

